
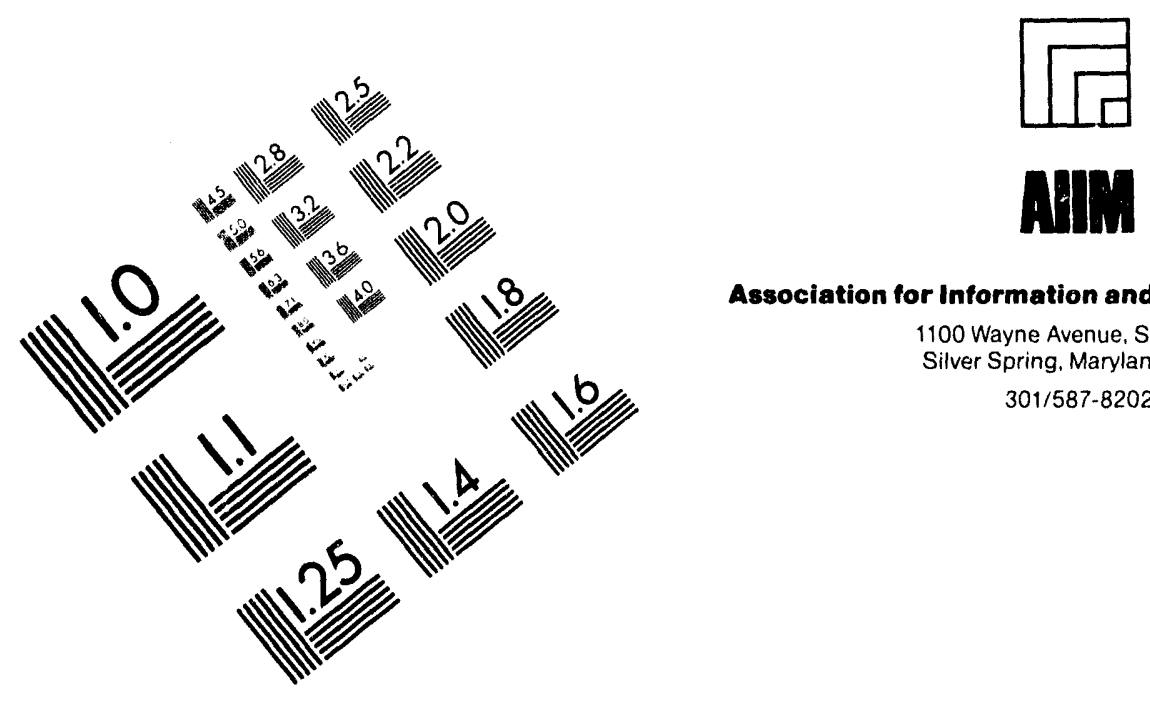

Association for Information and Image Management

1100 Wayne Avenue, Suite 1100

Silver Spring. Maryland 20910

301/587-8202

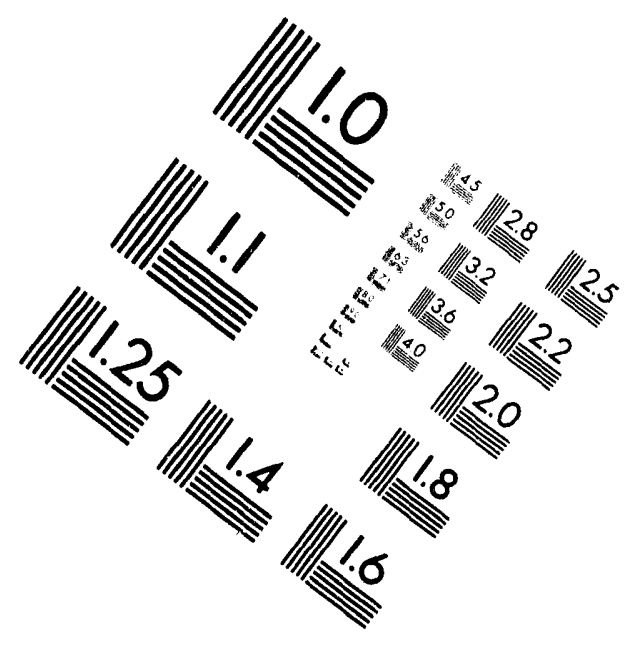

Centimeter

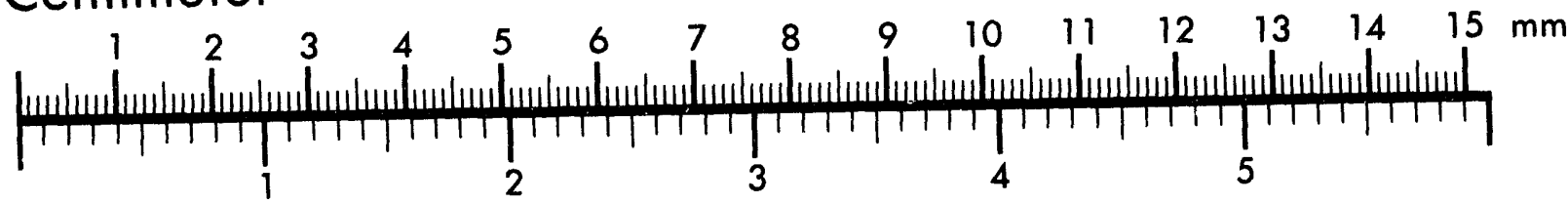
Inches
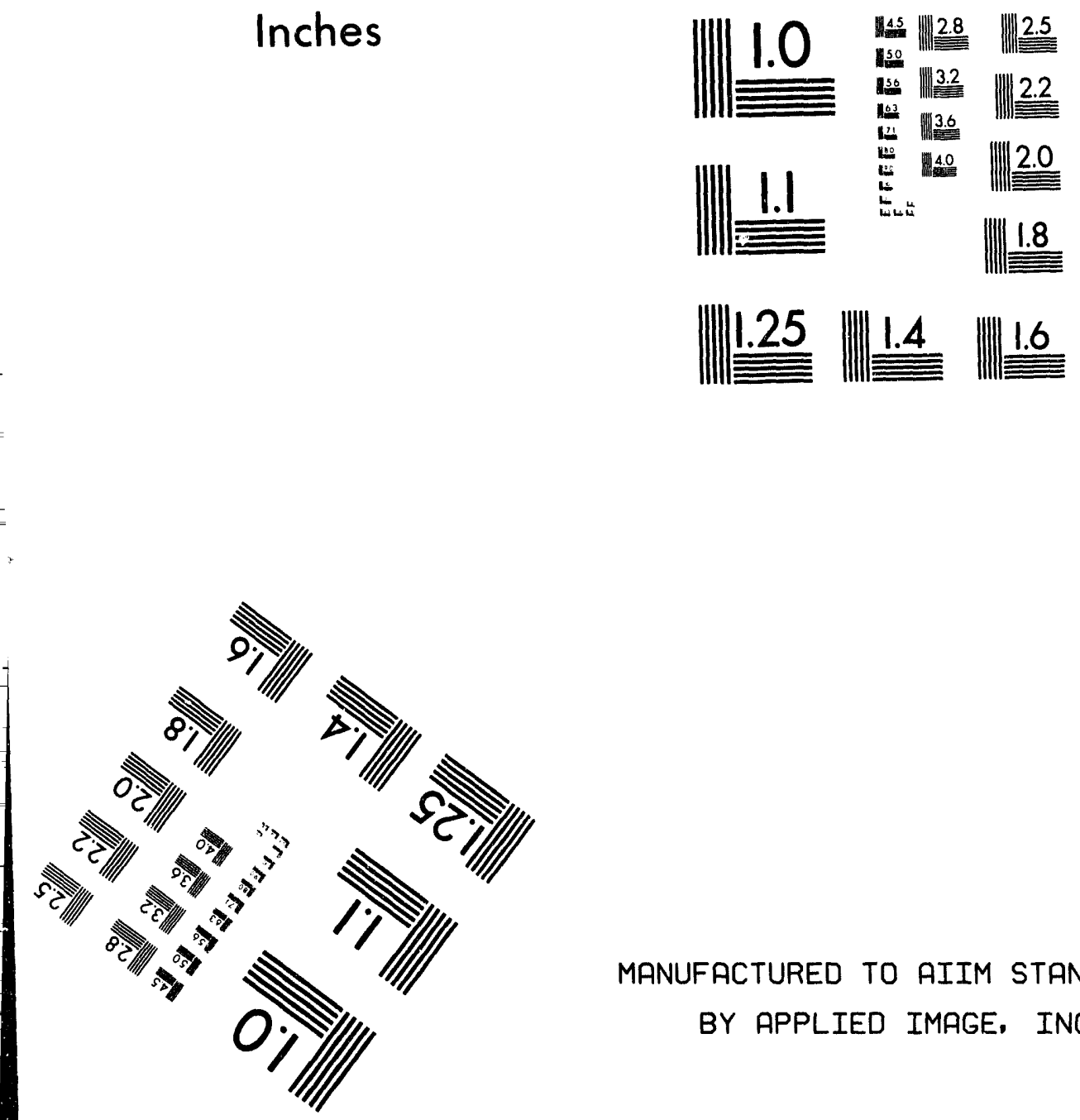

MANUFACTURED TO AIIM STANDARDS

BY APPLIED IMAGE, INC.

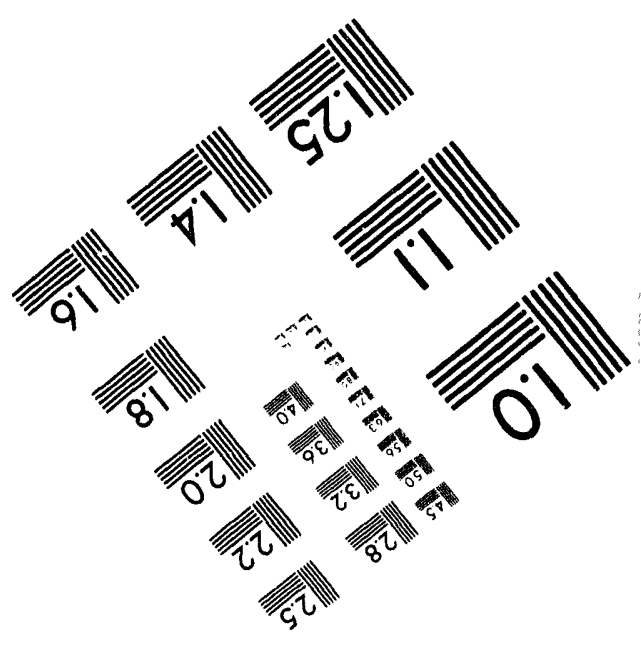



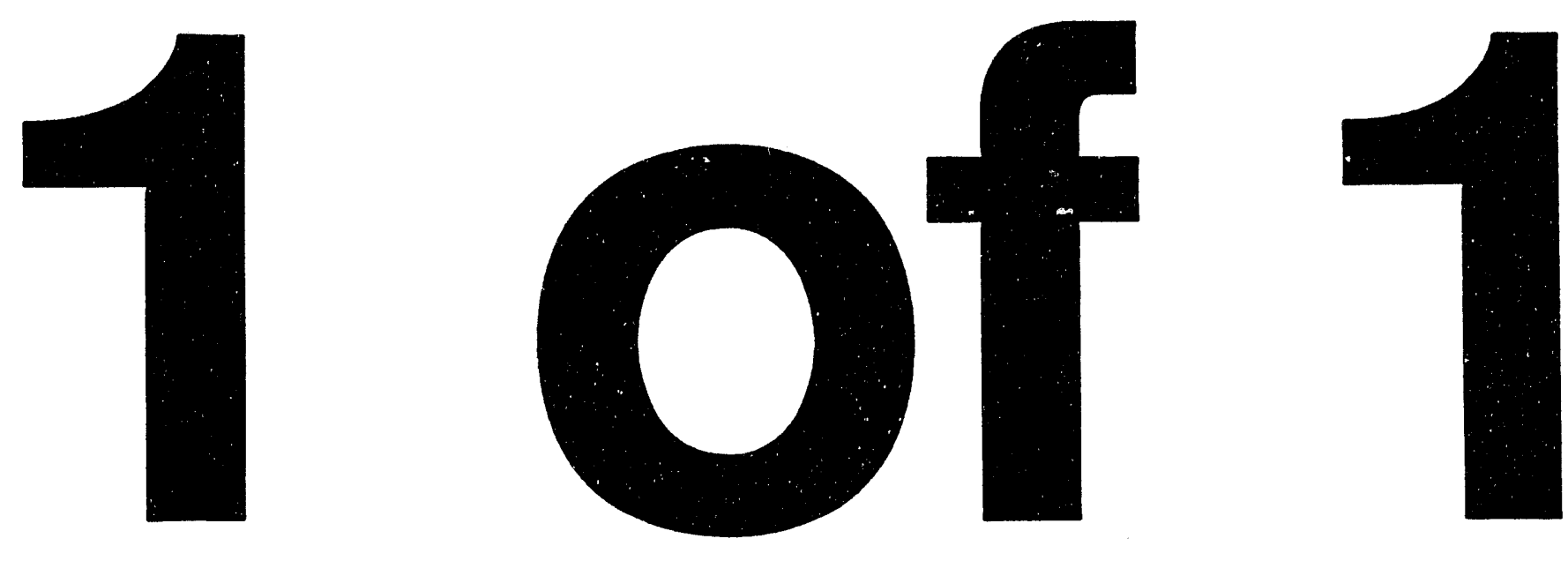
SAND93-4026

Distribution

Unlimited Release

Category UC-411

Printed December 1993

\title{
Trace Water Vapor Determination in Corrosive Gases by Infrared Spectroscopy
}

\author{
B.R. Stallard, R.K. Rowe, M.J. Garcia, and D.M. Haaland \\ Contamination Free Manufacturing Research Center \\ Sandia National Laboratories \\ Albuquerque, NM 87185 \\ L.H. Espinoza and T.M. Niemczyk \\ Department of Chemistry \\ University of New Mexico \\ Albuquerque, NM 87106
}

\begin{abstract}
A bstract
Delivery systems for corrosive gases may be compromised by contamination with even low levels of water vapor. Internal corrosion leads at first to increased particle counts in downstream fabrication tools and later to catastrophic failure of the piping. To extend the life of gas delivery systems and improve wafer yields, there is a need for an in-line monitor of $\mathrm{H}_{2} \mathrm{O}$ contamination. The goal of this project is to develop such an instrument, based on infrared spectroscopy, that has a detection limit of $30 \mathrm{ppb}$ or better and costs $\$ 50 \mathrm{~K}$ or less.

The first year's work, as reported herein, considered the application of Fourier transform infrared (FTIR) spectroscopy to $\mathrm{H}_{2} \mathrm{O}$ detection in $\mathrm{N}_{2}$ and $\mathrm{HCl}$. Using a modified commercial FTIR spectrometer and a long-path gas cell, a detection limit of about 10 ppb was demonstrated for $\mathrm{H}_{2} \mathrm{O}$ in $\mathrm{N}_{2}$ and $\mathrm{HCl}$. This includes about a factor of three improvement achieved by applying quantitative multivariate calibration methods to the problem. Absolute calibration of the instrument was established by determining the absorptivities of the prominent $\mathrm{H}_{2} \mathrm{O}$ bands between 3600 and $3910 \mathrm{~cm}^{-1}$. Methods are described to minimize the background moisture in the beam path of the instrument and to compensate for this source of error. The study includes the optimization of such variables as spectral region, detector type, resolution, cell type, and path length. Surprisingly, resolving the narrow $\mathrm{H}_{2} \mathrm{O}$ bands (FWHM $\approx 0.20 \mathrm{~cm}^{-1}$ ) is not necessary to achieve optimal sensitivity. In fact, optimal sensitivity is achieved at 2 to $4 \mathrm{~cm}^{-1}$ resolution, allowing the use of an inexpensive interferometer in the final instrument.

A much smaller, second generation instrument is described that will have a conservatively estimaied detection limit of $1 \mathrm{ppb}$. Since the present laboratory instrument can be duplicated in its essential parts for about $\$ 90 \mathrm{~K}$, it is quite realistic to project a cost of $\$ 50 \mathrm{~K}$ for the new instrument, given its shorter optical cell and smaller, low-resolution interferometer. In addition, we have designed an accessory for existing FTIR spectrometers that may be marketed for as little as $\$ 10 \mathrm{~K}$.
\end{abstract}

i 


\section{A cknowledgements}

The authors thank Dave Melgaard for adding the CLS algorithms to the PLSSNL software.

The authors also thank Ed Thomas for providing a number of insights in applying multivariate analysis. 


\section{Contents}

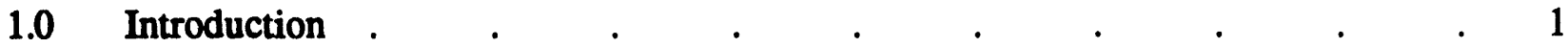

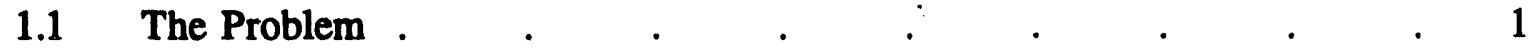

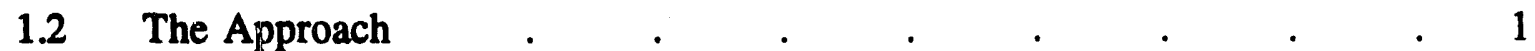

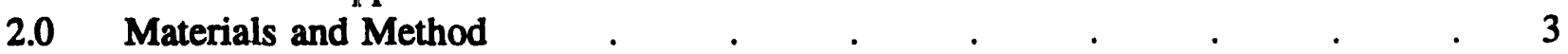

2.1 The Gas Handling Method . . . . . . . . 3

2.2 The Spectrometer . . . . . . . . . . 4

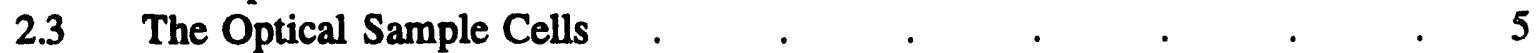

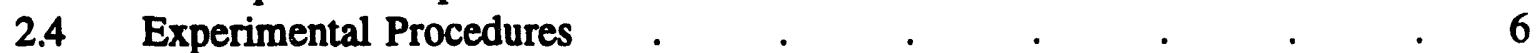

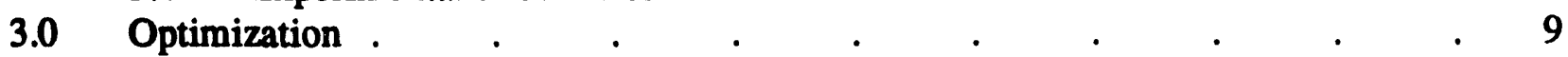

3.1 Choice of Detector and Spectral Region . $\quad . \quad$. $\quad . \quad 9 \quad 9$

3.2 Improvement with Multivariate Data Analysis . $\quad$. $\quad$. $\quad$. 10

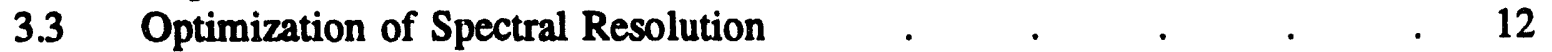

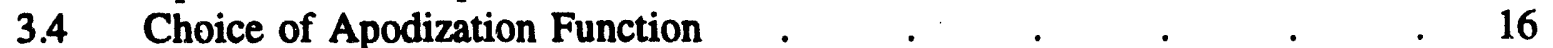

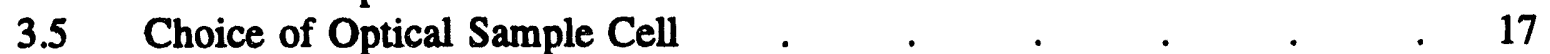

3.6 Choice of Length in Optical Sample Cell . $\quad . \quad \ldots \quad$. $\quad 18$

3.7 Summary of Standard Experimental Conditions _ . . . $\quad$. 19

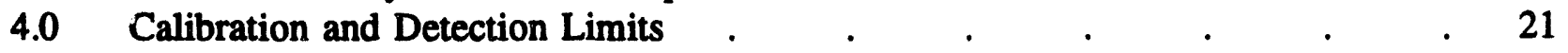

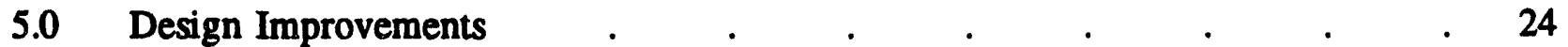

5.1 Description of a Mark II Prototype System . $\quad$. $\quad$. $\quad$. $\quad$. 24

5.2 Estimate of Mark II Performance . $\quad . \quad \ldots \quad \ldots \quad$. $\quad . \quad 25$

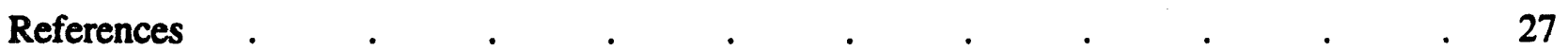

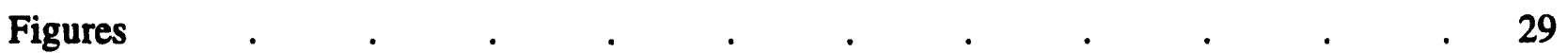

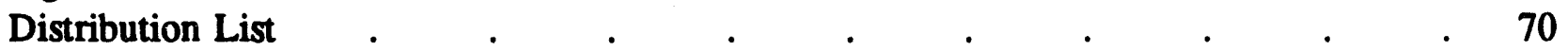


Intentionally Left Blank 


\subsection{Introduction}

\subsection{The Problem}

Delivery systems for corrosive gases may be compromised by contamination with even low levels of water vapor. Internal corrosion leads at first to increased particle counts in downstream fabrication tools and later to catastrophic failure. Once corrosion begins, its progress is difficult to arrest since the corrosion itself becomes a moisture source. To keep gas systems as moisture free as possible semiconductor manufacturers employ drying filters, usually just after the source of the process gas. Even so, the piping for corrosive gases may need to be rebuilt every 12 to 24 months.

To extend the life of gas delivery systems and improve wafer yields there is a need for an in-line monitor of $\mathrm{H}_{2} \mathrm{O}$ contamination. Presently there are several technologies costing $\$ 50 \mathrm{~K}$ or less that are capable of detecting trace water vapor as low as $50 \mathrm{ppb}$ in $\mathrm{N}_{2}[1,2]$. However, no one type of instrument has achieved universai acceptance. In particular, all have limited compatibility with corrosive gases such as $\mathrm{HCl}$ and $\mathrm{HBr}$. The goal of this project is to develop an in-line instrument based on infrared spectroscopy that is compatible with corrosive gases and has a detection limit of $30 \mathrm{ppb}$ or better. A cost of $\$ 50 \mathrm{~K}$ is the target for the new instrument.

\subsection{The Approach}

As an original project in the Contamination Free Manufacturing (CFM) Research Center, work began in August 1992. There are two additional CFM projects aimed at $\mathrm{H}_{2} \mathrm{O}$ detection in corrosive gases. One involves intracavity laser spectroscopy and the other uses solid state sensors made of porous silicon. In terms of cost and sensitivity, the prototype suggested by the present work falls between the two other projects. In addition, the present project is the closest to commercialization.

The first year's work, as reported herein, considers the application of Fourier transform infrared (FTIR) spectroscopy to $\mathrm{H}_{2} \mathrm{O}$ detection in $\mathrm{N}_{2}$ and $\mathrm{HCl}$. The application of diode laser modulation spectroscopy to this problem has also been proposed, but will not be discussed at this time. A report providing a preliminary comparison of these two approaches is available [3].

Earlier results leave no doubt that FTIR spectroscopy can be successfully used for trace $\mathrm{H}_{2} \mathrm{O}$ detection [4-7]. Important questions not fully answered in the literature are: 1) What parameters give the best instrumental performance? 2) What is the optimal way to analyze the data? 3) What detection limits can be achieved? 4) What is the absolute calibration? and 5) What instrumental design will produce the best results? Our current answers to these questions are the basis for this report. 
For the present, we assume that the calibrations for low levels of $\mathrm{H}_{2} \mathrm{O}$ in $\mathrm{N}_{2}$ and $\mathrm{HCl}$ are identical. This point has been addressed in the literature [4,5]. Given the conflicting results and the difficulty of the experiment, this question must be considered unresolved. If equating the two calibrations proves to be incorrect, the error in the $\mathrm{HCl}$ calibration is probably no worse than $30 \%$ [5]. This is not seen as a major problem at this stage of our instrument development. Investigations of the interaction of $\mathrm{H}_{2} \mathrm{O}$ with $\mathrm{HCl}$ and the effect of this interaction on the FTIR calibration will be described in a future report. 


\subsection{Materials and Method}

\subsection{The Gas Handling System}

The gas handling system is shown schematically in Fig. 1. It was designed and built in collaboration with Control Systems Incorporated of Rio Rancho, New Mexico. The tubing (mostly 1/4" diameter) is $316 \mathrm{~L}$ stainless steel, seamless, electropolished,and supplied by Valex Cutporation according to their 401 specification. Joints and bends are made with Cajon Micio-fit weld fittings. At points where periodic disassembly is required, Cajon VCR metal gasket fittings are used. Nupro diaphragm valves, model SS-DLBW4, are used throughout. The tubing and valves were assembled in a class 100 clean room following accepted procedures for ultra-high purity systems.

The moisture analyzer is an Aquamatic Plus by Meeco Incorporated. Moisture is absorbed on a hygroscopic material deposited on inert electrodes. A DC voltage applied to the electrodes electrolyzes the absorbed water into oxygen and hydrogen, which produces a current. According to Faraday's Law the measured current is proportional to the concentration of electrolyzed water. The stated accuracy is $\pm 20 \mathrm{ppb}$ or $\pm 2 \%$ of indicated reading, whichever is greater. A permeation tube from GC Industries is housed in a L'eau Pro moisture generator manufactured by Meeco Incorporated which provides control of temperature, pressure, and flow. The L'eau Pro was modified by removing the drying tube from its input port and substituting parts with increased $\mathrm{HCl}$ resistance where practical. Although the current system is designed to flow $\mathrm{HCl}$ directly past the permeation tube, a modification is planned to allow mixing wet $\mathrm{N}_{2}$ with dry $\mathrm{HCl}$.

The gas cabinet is a model GG100, two-cylinder cabinet from Air Products. The $\mathrm{HCl}$ source is Matheson semiconductor grade in a QF type bottle. The $\mathrm{H}_{2} \mathrm{O}$ content of the $\mathrm{HCL}$ is specified to be less than $30 \mathrm{ppm}$. The $\mathrm{HCl}$ dryer is a Waferpure gas purification system for chloride gases, model WGPS $030-2 \mathrm{C}$, from Millipore. The $\mathrm{N}_{2}$ source is boil off from a large liquid $\mathrm{N}_{2}$ tank located nearby. As measured by the Aquamatic Plus, the $\mathrm{H}_{2} \mathrm{O}$ content is typically $150 \mathrm{ppb}$. The $\mathrm{N}_{2}$ dryer is a Waferpure gas purification system for inert gases, model WGPS 030-31. Both Waferpure systems include particle filtering to $0.05 \mu \mathrm{m}$ and are specified to reduce moisture to less than $10 \mathrm{ppb}$.

The homemade scrubber bubbles $\mathrm{HCl}$ through a suitable aqueous basic solution before mixing residual gas with room air that is discharged through a dedicated roof top exhaust. The exhaust is monitored so that the operator can renew the scrubbing solution when the $\mathrm{HCl}$ content of the exhaust gas becomes unacceptably high. To avoid over taxing this part of the system, the flow rates of $\mathrm{HCl}$ are kept between 1 and 2 standard liters per minute (slm).

A vacuum system is attached to the three ports indicated in Fig. 1. The system consists of a diaphragm pump, a liquid nitrogen cooled adsorption pump, and an ion pump. Depending on the need, a vacuum between $10^{-1}$ and $10^{-6}$ torr. is used to condition the optical sample cells and the stainless steel tubing. Heat up to about $150^{\circ} \mathrm{C}$ may also be applied to 
these parts. Before switching the system from $\mathrm{HCl}$ to $\mathrm{N}_{2}$ the diaphragm pump is used to cycle the system several times between rough vacuum and purge. Also, on changing the $\mathrm{HCl}$ gas bottles, the diaphragm pump evacuates the system through the regulator to the bottle. This minimizes the burst of wet $\mathrm{HCl}$ when the valve is opened.

\subsection{The Spectrometer}

Fig. 2 shows the layout of the optical components on the optical table from Newport Corporation. The spectrometer is a Nicolet 800 spectrometer operated with a glowbar source and a $\mathrm{KBr}$ beamsplitter. A complete description of the instrumental operating parameters is given in Section 3. The collimated beam exits the side auxiliary port of the spectrometer and is directed to either of the two optical sample cells by the transfer optics. Two planar gold coated mirrors (not shown in the diagram) serve to align the beam accurately to the sample cells. The removable mirror in the transfer optics is an off-axis parabaloid that approximately matches the numerical aperture of the White cell (see Section 2.3).

The external detectors are either InSb, as shown, or HgCdTe (MCT), and can be interchanged between the cells. The detectors were purchased from Infrared Associates in liquid nitrogen cooled dewars and have a $2 \mathrm{~mm}$ diameter active area. A standard Nicolet dual-use preamplifier circuit board (ie., photovoltaic and photoconductive) is mounted on the base of the detector assembly so that the detector is readily coupled to the instrument's signal and control bus. As is customary, the InSb detector is used in a photovoltaic mode and the MCT detector in a photoconductive mode.

The removable mirror in the spectrometer is a standard component of the Nicolet $\mathbf{8 0 0}$ and serves to switch the beam between the external and internal detectors. The internal detector monitors the state of the instrument purge and provides a correction for the background $\mathrm{H}_{2} \mathrm{O}$ signal. More details regarding this procedure are given in Section 2.4. The internal deuterated triglycine sulfate (TGS) detector is the standard model available from Nicolet for this spectrometer. In spite of its poorer performance as compared to InSb and MCT, the TGS detector was chosen since it operates at room temperature. Hence, there is no need to disturb the purge of the instrument to add liquid nitrogen coolant.

The Nicolet spectrometer, as delivered, has a purge that is inadequate for $\mathrm{H}_{2} \mathrm{O}$ detection. To improve the purge, perforated tubes were attached to two of the three factory purge inlets and snaked around the spectrometer enclosure. Also, the entire spectrometer was placed in a polycarbonate box. The box consists of a sheet permanently attached to the underside of the spectrometer that extends about one inch beyond the factory enclosure. Utility feed-throughs are provided in the bottom sheet. A rectangular box, open at the bottom, lowers onto this base. It is latched tightly and sealed with dense foam. This design is recommended since normal use of the spectrometer is not compromised when the polycarbonate top is removed. The spectrometer, transfer optics, sample cell, and detector are connected in a leak-tight arrangement. When sealed, the enclosures are tested using an Airco 
Industrial Gases model 19AB leak tester. Imperfections in the enclosure are plugged with Apiezon $Q$ sealing putty. The spectrometer is purged by the boil off from a large liquid nitrogen tank that is run through a molecular sieve dryer at the point of use. The $\mathrm{N}_{2}$ stream contains about $100 \mathrm{ppb}$ of moisture when entering the spectrometer. A total flow of about 50 standard cubic feet per hour (scfh) through the dryer is partitioned between the spectrometer (35 scfh), the transfer optics (10 scfh), and the detector optics (5 scfh).

The drydown time of the enclosed spectrometer is illustrated in Fig. 3. The y scale for $\mathrm{H}_{2} \mathrm{O}$ concentration is in arbitrary classical least square (CLS) units that will be explained in Section 2.4. Using a sample cell with a path length of $8 \mathrm{~m}$ and a pressure of $1.37 \mathrm{~atm}$., one CLS unit is equivalent to $106 \mathrm{ppb}$. Fig. 3 shows that about 2 weeks of purging are required to reduce the background $\mathrm{H}_{2} \mathrm{O}$ level in the instrument to an equivalent of about 250 $\mathrm{ppb}$ in the sample cell. Most of the reported experiments were done with a moisture background in this range. The true concentration of $\mathrm{H}_{2} \mathrm{O}$ in the instrument is about $1.5 \mathrm{ppm}$. But the apparent concentration is less since the path length is shorter and the pressure lower in the instrument than in the cell. For comparison, Table 1 lists typical $\mathrm{H}_{2} \mathrm{O}$ concentrations in the environments of interest.

\begin{tabular}{|l|c|}
\hline Environment & Typical $\mathrm{H}_{2} \mathrm{O}$ Conc. \\
\hline atmosphere & $10,000 \mathrm{ppm}$ \\
\hline normal purge & $100 \mathrm{ppm}$ \\
\hline excellent purge & $1 \mathrm{ppm}$ \\
\hline
\end{tabular}

Table 1 - Typical $\mathrm{H}_{2} \mathrm{O}$ concentrations.

\subsection{The Optical Sample Cells}

As seen in Figs. 1 and 2, there are two optical sample cells in experimental set up. The White cell (see Fig. 4) was purchased from Infrared Analysis Incorporated. It is of conventional design with 4" diameter optics enclosed in a 6" diameter Pyrex tube with anodized aluminum end caps. It has a numerical aperture of 0.125 . Forty passes between the gold coated mirrors give a fixed pathlength of $22 \mathrm{~m}$. The Axiom cell (see Fig. 5) was purchased from Axiom Analytical Incorporated. It has eight nickel-coated brass tubes of $2 \mathrm{~m}$ length and 1.25" diameter (only four tubes are shown in the figure for simplicity). The folded, single pass design has two $45^{\circ}$ mirrors at the end of each tube. The mirror assemblies can be repositioned on the tubes to give a configuration of $2,4,6$, or 8 tube lengths. Heating tape from Clayborn Lab was permanently affixed to four tubes allowing those sections of the Axiom cell to be heated to about $150^{\circ} \mathrm{C}$, even with the cooling effect of a $10 \mathrm{sim}$ purge. 


\subsection{Experimental Procedures}

Interferograms were transferred from the Nicolet SX computer to a $486 / 50$ personal computer, where they were translated to the proper format by the PCIR software (Nicolet). The principle manipulations were done within Lab Calc (Galactic Industries), including apodization, zero-filling, Fourier transformation, multivariate analysis, etc. A large number of custom programs were written in the Array Basic language for use with Lab Calc. Most notable is the expansion of the general multivariate analysis program PLSSNL [8] to include the algorithm for classical least squares (CLS) [9].

Absorption spectra were produced from interferograms without relying on an independent background spectrum. Instead, each interferogram was used to produce its own background spectrum by removing the high frequency information before performing the Fourier transform. Specifically, the intensity of each point in the interferogram was weighted by a Gaussian centered at the zero point of the mirror travel, with $\sigma=30$ distance units, where 7900 distance units make up the entire interferogram. This procedure is an effective route to an absorption spectrum when a true background spectrum (ie. zero concentration of sample) is unobtainable. It is particularly applicable when the spectral features are sharp relative to variations in the single beam spectrum.

Zero-filling, which increases the number of points in the absorption spectra by a factor of two, was used to improve the appearance of the spectra. After zero-filling, a spectrum at 1 $\mathrm{cm}^{-1}$ has a point spacing of $0.25 \mathrm{~cm}^{-1}$. The effect of zero-filling on the CLS computation seems to be negligible, although no rigorous demonstration of this point was attempted.

Fig. 6 shows an $\mathrm{H}_{2} \mathrm{O}$ spectrum at a resolution of $1 \mathrm{~cm}^{-1}$. The ten indicated regions, encompassing the strongest peaks, were chosen for the CLS computation. In each region the CLS algorithm fits the unknown spectrum with the reference spectrum and a linear baseline. This gives a value for the intensity of the unknown spectrum, relative to the reference spectrum in each region. A weighted average yields the final concentration result, where the weighting factors are inversely proportional to the square of the spectral residuals.

Since changes in absorption band width and position affect the CLS calculation, a separate reference spectrum was acquired for each set of experimental conditions. To insure a high SNR for the reference spectra, typically the $\mathrm{H}_{2} \mathrm{O}$ concentration was about $1 \mathrm{ppm}$ and the collection time about 1 hour. Arbitrarily, one of the reference spectra was given a concentration value of 100 units; hence the origin of the arbitrary CLS unit used throughout this paper. All other reference spectra were placed on the same scale by calculating the relative value of the peak areas in the ten regions indicated in Fig. 6.

Fig. 7 illustrates the algorithm used to arrive at the CLS estimate of the $\mathrm{H}_{2} \mathrm{O}$ concentration in the sample. The CLS estimate of the background $\mathrm{H}_{2} \mathrm{O}$ is derived from the TGS detector, while the CLS estimate of the background plus sample $\mathrm{H}_{2} \mathrm{O}$ is derived from the InSb detector. The CLS estimate for the TGS detector is scaled to account for the path 
difference between the two measurements that is external to the cell (see the next paragraph). Finally, a simple subtraction gives the CLS estimate for the sample only.

To determine the scaling factor appropriate to correct the TGS measurement of the background $\mathrm{H}_{2} \mathrm{O}$, the optical cell was removed from the beam path of the InSb detector and replaced with a short section of Axiom light pipe. The entire InSb path was left open to the instrument purge. Repeated CLS measurements of the $\mathrm{H}_{2} \mathrm{O}$ concentration in the TGS and InSb beam paths were made over a period of seven days. The ratio of InSb to TGS is plotted in Fig. 8. The mean of 1.103 is the scaling factor that accounts for the difference in path lengths. A measure of the precision of this determination is the standard error of estimate (SEE). It is the RMS of the errors relative to the model, which, in this case, is a second order polynomial fit to the data (the solid line). The unitless SEE of 0.018 is completely accounted for by the precision of the TGS and InSb measurements (see Section 4).

Moreover, the standard errors of the polynomial coefficients describing the curve in Fig. 8 are not statistically different from zero. Therefore, the fit is not distinguishable from a horizontal straight line. To account for a somewhat different path length when the White cell is used, the scaling factor is found to be 1.130 .

Conditioning the Axiom cell was necessary to achieve reproducible calibrations of $\mathrm{H}_{2} \mathrm{O}$ in $\mathrm{N}_{2}$ in the range 40 to $400 \mathrm{ppb}$. Before each calibration run, the gas cell and the entire gas handling system were purged for about three days to reach a moisture level of about $30 \mathrm{ppb}$ as read on the Aquamatic Plus. During this purge the Axiom cell was heated to about $150^{\circ} \mathrm{C}$. After cooling, adjusting the pressure, and equilibrating the cell for about two hours, the InSb and TGS interferograms were collected and the data analyzed following the scheme of Fig. 7. Subsequent points at different levels of $\mathrm{H}_{2} \mathrm{O}$ were preceded by a heated purge of about five hours. The desired $\mathrm{H}_{2} \mathrm{O}$ concentrations were obtained using the moisture generator by varying the flow of nitrogen past the permeation device. The order of collecting the various $\mathrm{H}_{2} \mathrm{O}$ levels was randomized. In principle, either the moisture generator or the Aquamatic Plus may be used as a standard for the spectrometer calibration. In practice, much higher precision was obtained with the Aquamatic Plus. Therefore, only these results are reported.

Another important procedure involves measuring the spectral noise to signal ratio (NSR) by means of $100 \%$ lines. A $100 \%$ line is the ratio of two single beam spectra taken without intervening delay, under identical conditions. Fig. 9 gives two examples of $100 \%$ lines where the NSR as a function of resolution is under investigation. These results are part of the data incorporated into Fig. 19. The RMS deviation from the mean in a $100 \%$ line is $\sqrt{2}$ times the NSR. Using a $\mathbf{i 0 0 \%}$ line in this way can easily produce erroneous results when, as in the present case, an absorber is present in the spectral region of interest. There is a danger that noise may be confused with imperfect cancellation of real spectral features. To insure the validity of NSR values derived from the $100 \%$ lines, we thoroughly purged the spectrometer to minimize the $\mathrm{H}_{2} \mathrm{O}$ signal and confirmed the cancellation of the $\mathrm{H}_{2} \mathrm{O}$ spectrum by plots such as Fig. 10. Twenty spectra of 50 scans each were collected. Spectrum \#1 was ratioed to \#2 to produce the first point. Spectra \#1+\#3 were ratioed to \#2+\#4 to produce 
the next point, and so on. The RMS of the $100 \%$ lines follows the expected dependence, (\# scans) $^{-1 / 2}$. This is a convincing demonstration that the noise does not have a systematic component that may be related to non-canceled $\mathrm{H}_{2} \mathrm{O}$ bands. 


\subsection{Optimization}

\subsection{Choice of Detector and Spectral Region}

The strongest $\mathrm{H}_{2} \mathrm{O}$ absorption bands in the mid-infrared are centered at approximately 1600 and $3800 \mathrm{~cm}^{-1}$. Fig. 11 shows that the magnitude of the individual absorption lines within these two bands is roughly equivalent. Therefore, the selection of a spectral region was principally contingent upon the characteristics of the available detectors.

Three infrared detectors, TGS, MCT, InSb, were considered for this experiment. Fig. 12 shows their specific detectivity values $\left(\mathrm{D}^{*}, \mathrm{~cm} \mathrm{~Hz}^{\text {th }} \mathrm{W}^{-1}\right)$ [10], which can be interpreted as the SNR at the output of the detector when $1 \mathrm{~W}$ of radiant power is incident on a $1 \mathrm{~cm}^{2}$ detector surface with a $1 \mathrm{~Hz}$ bandwidth. Only typical values are shown, while, in practice, a broad range may be observed. Fig. 13 displays the single beam spectrum of each detector, which indicates its effective relative spectral sensitivity as influenced by the glowbar source and the spectrometer optics. The MCT single beam spectrum in Fig. 13 has a high $\mathrm{H}_{2} \mathrm{O}$ signal to clearly indicate the position of these bands.

Although the TGS has a low D*, it is sensitive to the entire mid-infrared spectral range and does not require cooling by liquid nitrogen. For these reasons, a TGS detector is used within the enclosure of the spectrometer, where access is severely restricted to maintain the integrity of the purged environment.

Given easy access to the external detector, the two more sensitive, liquid nitrogen cooled detectors were considered for this application. As shown in Fig. 12, the operating range of InSb is about 1,800 to $8,000 \mathrm{~cm}^{-1}$, with a peak $\mathrm{D}^{*}$ of approximately $1 \times 10^{11}$ $\mathrm{cm} \mathrm{Hz}^{1 / 2} \mathrm{~W}^{-1}$ at $2,000 \mathrm{~cm}^{-1}$. The InSb detector is effectively limited to measurements of the $3800 \mathrm{~cm}^{-1} \mathrm{H}_{2} \mathrm{O}$ band. Also shown in Fig. 12, are the spectral characteristics for an MCT detector, which is sensitive to wavelengths between about 600 and $5,000 \mathrm{~cm}^{-1}$, and has a peak $D^{*}$ of approximately $1 \times 10^{10} \mathrm{~cm} \mathrm{~Hz}^{1 / 2} \mathrm{~W}^{-1}$ at $850 \mathrm{~cm}^{-1}$. While the operating range of the MCT detector encompasses both of the $\mathrm{H}_{2} \mathrm{O}$ absorption bands of interest, Fig. 13 shows that there is approximately a $20 \%$ improvement in sensitivity in the $1600 \mathrm{~cm}^{-1}$ region relative to the $3800 \mathrm{~cm}^{-1}$ region.

The relative performance of an MCT detector operating at $1600 \mathrm{~cm}^{-1}$ and an InSb detector operating at $3800 \mathrm{~cm}^{-1}$ can be estimated as follows. Assuming that the dominant noise source in an FTIR spectrum is signal-independent detector noise, and assuming a unit detector area and unit bandwidth, the SNR for an InSb detector operating at $3800 \mathrm{~cm}^{-1}$ is proportional to the $D^{*}$ of about $6 \times 10^{10} \mathrm{~cm} \mathrm{~Hz}^{1 / 2} \mathrm{~W}^{-1}$. The $\mathrm{D}^{*}$ at $3800 \mathrm{~cm}^{-1}$ for the MCT detector is about $5 \times 10^{9} \mathrm{~cm} \mathrm{~Hz}^{1 /} \mathrm{W}^{-1}$, or about 12 times lower. Using the $1600 \mathrm{~cm}^{-1}$ spectral region improves the MCT performance by $20 \%$, thereby reducing the SNR advantage of the InSb detector to 10:1. 
An experimental validation of these results was performed using a Nicolet 800 spectrometer, together with the MCT and InSb detectors supplied with the spectrometer. The two detectors have the same active area, and the electronic bandpass of the spectrometer was held constant. By comparing the RMS fluctuation of the respective $100 \%$ lines in the 3800 $\mathrm{cm}^{-1}$ region, it was determined that the SNR of the InSb detector was 19 times greater than the SNR of the MCT detector. As mentioned previously, a $20 \%$ increase in the SNR is realized by using the MCT detector for analysis in the $1600 \mathrm{~cm}^{-1}$ region. This reduces the advantage of InSb over MCT to $16: 1$, which, considering the variability common from detector to detector, is consistent with the 10:1 ratio predicted above.

These results show that an InSb detector outperforms an MCT when operated at the same low light level. However, a caveat that was not considered in the foregoing analysis, is that the InSb detector saturates much more readily than the MCT detector. In the Nicolet spectrometer used at $1 \mathrm{~cm}^{-1}$ resolution, the total available light intensity may be used by the MCT detector while the light must be attenuated by about a factor of 30 to use the InSb detector. In such cases, the InSb detector's advantage of a higher $D^{*}$ may be more than offset by the fact that the MCT detector can be used at higher light levels. If we use the suggested factor of 30 as a difference in light levels, then the SNR of an MCT detector operated in the $1600 \mathrm{~cm}^{-1}$ region (at high light level) may be about 2 (ie., 30/16) times greater than the SNR of an InSb detector operated in the $3800 \mathrm{~cm}^{-1}$ region (at low light level).

In summary, the use of an InSb detector is highly favored for low light level applications while an MCT detector is slightly favored for high light level applications. The low light situation was often the case for the experiments undertaken during this project. For this reason, and to collect consistent and comparable data, it was decided to primarily use the InSb detector. Also, Section 5.2 describes an improvement in instrumental design which will clearly make InSb the preferred detector.

\subsection{Improvement with Multivariate Data Analysis}

In general, a multivariate technique is expected to perform better than a univariate method. There exist a variety of multivariate techniques for spectroscopic analysis including classical least squares (CLS) [9], partial least squares [11], principal component regression [12], and others [13]. Our choice of analytic technique was driven by the relative simplicity of the required spectroscopic model. We assume that the relationship between the concentration of $\mathrm{H}_{2} \mathrm{O}$ and the absorbance is linear, that the pertinent features of the absorption spectrum are due to known components, and that pure-component spectra are available. Based upon these assumptions, CLS was chosen as the appropriate analytic method.

The degree of improvement of the CLS method over the univariate technique can be quantified by comparing the standard deviation of the concentration estimates produced by the two methods. The univariate concentration estimate is the height of a chosen absorption peak in the sample spectrum relative to that of the same peak in the reference spectrum. The height of a peak within the sample spectrum can be calculated as the difference between the 
value at the peak of an absorption line, $A_{\mathrm{sm}}$, and a nearby, off-peak (i.e., baseline) point, $A_{\mathrm{s} 0}$. This difference, $\Delta A_{2}$, is then ratioed to the difference of the values at the same two points in a reference spectrum, $\Delta \mathrm{A}_{\mathrm{T}}$, which may also be called the pure component or model spectrum. The result is an estimate of the $\mathrm{H}_{2} \mathrm{O}$ concentration in the sample as a fraction of the concentration in the reference spectrum:

$$
\hat{\mathbf{k}}_{\text {uni }}=\Delta \mathbf{A}_{\mathbf{z}} / \Delta \mathbf{A}_{\text {, }} \text {, }
$$

where the caret indicates an estimate of the true value. Implicit in this model is the assumption that the Beer's law applies (i.e., the absorbance of the sample is linearly proportional to the concentration of the sample), and that there are no linear or higher order baseline shifts of the sample spectrum relative to the reference spectrum.

To ascertain the precision of this estimate, several assumptions must be made about the spectral noise. First, an assumption is made that the reference spectrum has no noise. Second, it is assumed that the noise in the sample spectra is uncorrelated from spectral element to spectral element, and is additive, zero-mean, and normally distributed. Finally, the assumption is made that the variance of the noise at each element within the sample spectrum is a constant, $\sigma_{z}{ }^{2}$. This last assumption is strictly true only in the transmission spectra, but is a good approximation for the noise in the absorption spectrum when weak absorptions are considered, such as in this project. Under these conditions, the standard deviation of the univariate estimator given in Eq. 1 is

$$
\sigma_{k, \text { uai }}=2^{1 / 2} \sigma_{2} / \Delta A_{1}
$$

The CLS concentration estimate that is used in this work is based upon the following model for the data:

$$
A_{\mathrm{ai}}=\mathrm{a}+b v_{\mathrm{i}}+k A_{\mathrm{ri}}+\mathrm{e}_{\mathrm{si}}
$$

where $\mathrm{a}$ and $\mathrm{b}$ are the parameters used to model an assumed linear baseline component of the sample absorption spectrum, $v_{i}$ is the frequency at the ith element of the sample spectrum, $e_{s i}$ is the random noise value at the same ith element, and the other parameters are the same as defined in the univariate analysis. The properties of the noise are also assumed to be the same as the univariate case: additive, normally-distributed, zero-mean, and uncorrelated between spectral elements.

Under these conditions, least-squares estimates of the three parameters can be found from the following relationships [9]:

$$
\begin{aligned}
& \hat{\mathbf{k}}=\left(c_{11} c_{23}-c_{12} c_{13}\right) /\left(\mathrm{c} \mathrm{c}_{11}\right), \\
& \hat{b}=\left(c_{22} c_{13}-c_{12} c_{23}\right) /\left(\mathrm{c} \mathrm{c}_{11}\right), \\
& \hat{a}=\bar{A} s-\hat{b} \bar{v}-\hat{\mathbf{k}} \bar{A} s,
\end{aligned}
$$


where a bar denotes the mean over the $\mathbf{N}$ points in the spectral region being evaluated. The various "c" coefficients consist of combinations of sums of the spectral values as follows:

$$
\begin{aligned}
& c_{11}=\Sigma v_{i}^{2}-\left(\Sigma v_{i}\right)^{2} / N \text {, } \\
& c_{12}=\Sigma v_{i} A_{r i}-\left(\Sigma v_{i}\right)\left(\Sigma A_{r i}\right) / N \text {, } \\
& c_{13}=\Sigma v_{i} A_{a i}-\left(\Sigma v_{i}\right)\left(\sum A_{a i}\right) / N \text {, } \\
& c_{22}=\Sigma A_{r i}{ }^{2}-\left(\Sigma A_{r i}\right)^{2} / N \text {, } \\
& c_{23}=\sum A_{r i} A_{i i}-\left(\sum A_{i i}\right)\left(\sum A_{s i}\right) / N \text {, } \\
& c=\left(c_{11} c_{22}-c_{12}{ }^{2}\right) / c_{11} \text {. }
\end{aligned}
$$

The standard deviation of the CLS concentration estimate, $\sigma_{k, ~ a b}$, is given by,

$$
\sigma_{k, \text { ak }}=\sigma_{s} / \sqrt{ } c
$$

The improvement of the CLS estimator relative to the univariate estimator is simply given by the ratio of the two estimator standard deviations:

$$
\sigma_{k, \text { ch }} / \sigma_{k, \text { uni }}=\Delta A_{1} /(2 \mathrm{c})^{k / .}
$$

The exact value of this ratio depends on the spectrum under analysis and the operating parameters of the spectrometer. The ideas presented above will be further developed in the next section.

Fig. 14 illustrates the increase in the SNR of the CLS over the univariate estimator for a series of resolutions at a fixed collection time. The data are experimental where the SNR is taken as the SEE of $\mathbf{4 0}$ measurements of a slowly varying sample of $\mathrm{H}_{2} \mathrm{O}$ in $\mathrm{N}_{2}$, as in Figs. 37 and 38. In processing the interferograms, the following parameters were used to conform to the theory developed in the next section: boxcar apodization, no zero-filling, and spectral region 3788 to $3910 \mathrm{~cm}^{-1}$ without peak selection. Equivalent plots calculated with the standard set of parameters (see Section 3.7) show only small differences. The univariate results are derived from the absorptior band at $3837.87 \mathrm{~cm}^{-1}$ and based on an analogous calculation of the SEE of $\mathbf{4 0}$ repeated measurements. Experimentally, we see that the improvement in precision of the CLS over univariate analysis is from 2 to 5 times. The dependence of SNR on resolution as revealed in Fig. 14 is the subject of the next section.

\subsection{Optimization of Spectral Resolution}

A series of numerical analyses was performed to better understand the way that spectrometer resolution influences the measurement precision. Although the ultimate goal of these analyses is to optimize the system resolution for the case of a CLS estimation technique applied to realistic spectral data, it is informative to first consider the case of a simple univariate concentration estimate based upon data produced by an ideal system used to collect a single scan. Using the form of the univariate estimator described in the previous section, 
Eqs. 1 and 2 can be combined to produce an expression for the SNR of the univariate estimate:

$$
\begin{aligned}
& \mathrm{SNR}_{\text {uai }}=\left\langle\hat{\mathrm{k}}_{\text {uni }}\right\rangle \Delta \mathrm{A}_{1} /\left(2^{\mathrm{k}} \sigma_{\mathrm{s}}\right) \text {, } \\
& =\Delta A_{1} \Delta A_{1} /\left(2^{H_{1}} \Delta A_{1} \sigma_{1}\right) \text {, } \\
& =\Delta A_{1} /\left(2^{1 / 2} \sigma_{1}\right) \text {, }
\end{aligned}
$$

where $<\hat{\mathrm{k}}_{\mathrm{umi}}>$ designates the mean of the concentration estimate. Assuming that the dominant noise in the FTIR spectrometer is signal-independent detector noise and that the optical throughput of the instrument is held constant, then the variance of the noise in a single spectrum, $\sigma_{1}{ }^{2}$, can be related to the noise variance in the interferogram, $\sigma_{\text {int }}{ }^{2}$, using Parseval's theorem:

$$
\Delta v \sigma_{i}^{2}=\Delta x \sigma_{\text {int }}^{2}
$$

where $\Delta v$ is the sample spacing in the spectrum and $\Delta x$ is the sample spacing of the interferogram. In this instance, the spectral resolution, $R$, is simply twice the width of the spectral elements. Therefore, the spectral noise is given by,

$$
\sigma_{\mathrm{s}}=(2 \Delta \mathrm{x})^{\mathrm{h}} \sigma_{\mathrm{int}} / \mathrm{R}^{\mathrm{h}}
$$

Furthermore, if the spectral measurement region is held constant, then $\Delta \mathrm{x}$ will be constant. It is also reasonable to assume that the noise in the interferogram is constant for a given spectrometer and set of operating parameters. Under these conditions, the standard deviation of the spectral noise is simply proportional to the inverse square root of the resolution, and the SNR associated with the univariate concentration estimate is:

$$
\mathrm{SNR}_{\mathrm{uai}}=\Delta \mathrm{A}_{8} \mathrm{R}^{1 / 2} /\left(4 \Delta \mathrm{x} \sigma_{\mathrm{int}}{ }^{2}\right)^{1 / 2}
$$

The line with solid triangles in Fig. 15 is a plot of Eq. 18 as a function of the system resolution. The data were generated using a numerical simulation of an FTIR spectrometer with a spectral range of $0-7900 \mathrm{~cm}^{-1}$, a single scan, ideal noise characteristics (as given by Eq. 17), no apodization or zero-filling of the data, and viewing a single, isolated absorption line at $3837.87 \mathrm{~cm}^{-1}$. The absorption line was selected from the HITRAN database [14] and the line profile (i.e., Voigt) parameters were adjusted to correspond to an experimental condition of $296 \mathrm{~K}, 1.37 \mathrm{~atm}$, and air-broadened (FWHM $\approx 0.2 \mathrm{~cm}^{-1}$ ). This figure indicates that the optimum resolution of an ideal FTIR spectrometer viewing such an absorption line is approximately $1.0 \mathrm{~cm}^{-1}$, which implies a condition in which the absorption feature is under resolved.

The functional form of the univariate estimator shown in Fig. 15 can be better understood by looking at the numerator and denominator of Eq. 15 separately. The line with solid circles in Fig. 16 shows the peak height, $\Delta \mathbf{A}_{\mathbf{s}}$, as a function of resolution. As expected, the peak height increases as the system resolution becomes narrower until the absorption line 
is nearly resolved, at which point the peak height begins to level off. Conversely, the noise, $\sigma_{3}$, is proportional to the inverse square root of the resolution and increases sharply at narrow resolutions, as shown by the line with solid triangles in Fig. 16. Therefore, while the observed peak height increases slightly as the system better resolves the absorption line, the noise level increases dramatically. The net effect is that the system can achieve a better level of performance at a resolution in which the peak height is slightly diminished, but the noise level is greatly reduced.

The previous results have all been based upon the assumption of a single scan. However, these results can be easily adjusted to the situation in which $M$ scans are collected and the interferograms are averaged before the transformation that produces the absorption spectrum. In this case, the standard deviation of the noise in the interferogram, $\sigma_{\text {int }}$, will be reduced by a factor of $M^{\text {h }}$, which will reduce the noise in the spectrum by the same factor, as shown in Eq. 16. Therefore, when the FITR data consist of $M$ scans, the univariate SNR is given by:

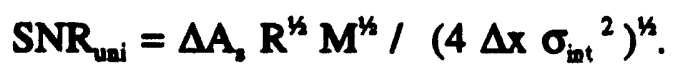

It is easy to see that if the number of scans is held constant with respect to the system resolution, the same functional forms shown in Fig. 15 will apply. However, another common operating mode of an FTIR system -- and the one under which most of the experimental data in this study were taken -- is the fixed-time condition, in which the number of scans that are collected is a function of the resolution of the spectrometer. Different spectral resolutions require different amounts of mirror travel, which, in turn, affects the number of scans that can be made in a given time. The number of scans that an ideal instrument with a constant scanning speed (i.e., scanning distance per unit time) can collect in a fixed time is linearly proportional to the spectral resolution [15]. The functional form of the SNR of the univariate estimator in a system operating in a fixed-time mode is shown in Fig. 17. All of the assumed parameters, except the number of scans, are identical to those used to generate Fig. 15. As shown by the figure, the best performance of an ideal spectrometer operating in fixed-time mode and viewing a single, isolated absorption line can be achieved with broad resolution values (i.e., "poor" resolution). This is because the noise in the system is greatly reduced via two effects: the decreased level of noise in a low-resolution spectrum (Eq. 17), and by the large number of scans that can be collected and averaged, further reducing the noise by the square root of the number of scans (Eq. 19).

The CLS estimator can be analyzed in a similar manner to the preceding univariate analysis. The SNR of the CLS concentration estimate can be found from Eqs. 4, 13, 17, and by including the effect of multiple scans:

$$
\operatorname{SNR}_{\mathrm{cls}}=\left(c_{11} c_{23}-c_{12} c_{13}\right) R^{1 / 2} M^{1 / 2} /\left(c_{11}(\Delta x)^{1 / 2} \sigma_{\text {int }}\right) \text {. }
$$

The lines with the solid circles in Figs. 15 and 17 plot $S_{N R_{c l s}}$ as a function of resolution for a fixed number of scans and a fixed time, respectively, for the same system model as used for 
the previous univariate analysis. As expected, the CLS estimator outperforms the univariate estimator in both the fixed-number-of-scans and fixed-time modes, especially in the region in which the absorption feature is nearly resolved.

While the previous analyses are useful to understand the trends of the univariate and CLS concentration estimators, it is not realistic to evaluate the performance of the estimators using a single absorption line. To expand the numerical comparison, the HITRAN database was used to generate a water vapor absorption spectrum corresponding to the conditions of $296 \mathrm{~K}$ and $1.37 \mathrm{~atm}$. Fig. 18 snows a portion of one such simulated spectrum, which includes the region of interest. The spectrum was further processed to simulate the scans of an FTIR spectrometer at various resolutions with no zero-filling or apodization. The data within the region of interest were then extracted and processed according to Eq. 20 for both the fixednumber-of-scans and fixed-time modes of data collection.

The Nicolet spectrometer differs from an ideal instrument in two major respects: the dependence of spectral noise and the dependence of scan time on resolution. These non-ideal characteristics of the spectr umeter were included in the numerical model to obtain more realistic results. First, tb: functional relationship between the noise and resolution was determined from 1 now lines collected at a range of resolutions. Fig. 19 shows that the noise is proportional to $R^{-0.63}$, instead of the ideal value of $R^{-0.50}$. This indicates a more rapid increase in noise than expected at narrow resolution. The reason for this is not known, but may be due to increased mirror vibrations at large displacements. Second, Fig. 20 shows the number of scans completed in 10 minutes at a variety of resolution settings. The discontinuity between the resolutions of 1 and $2 \mathrm{~cm}^{-1}$ is a consequence of changing from single-sided to double-sided interferograms. For the two regions, Fig. 20 shows the best linear fits to the data. Note that the appearance of linearity is lost because of the $\log$ scale. Clearly, the spectrometer does not do as well as the ideal spectrometer which doubles the number of scans in a fixed time when $R$ doubles. The real spectrometer has an increased proportion of overhead time as the scans get shorter. The trends in Fig. 20 were modeled as 4th order polynomials (not shown) in the following simulations.

Figs. 21 and 22 show the estimator SNR's for a fixed-number-of-scans and a fixedtime, respectively. The conditions are: broad analysis region, realistic noise characteristics, and nonlinear time-per-scan characteristics. The figures indicate that the CLS estimator has an optimum operating resolution: $0.5 \mathrm{~cm}^{-1}$ for fixed-number-of-scans, and $8 \mathrm{~cm}^{-1}$ for fixedtime. It is the latter figure that is most nearly comparable to the operating mode that was chosen for the experiments. The theoretical results in Fig. 22 generally have the same form as the experimental results in Fig. 14. Discrepancies between theory and experiment are the subject of further investigation. At this point it is believed that a nonlinear baseline component present in the experimental spectra contributes to the differences.

Without the benefit of the present analysis, it might be tempting to use a resolution sufficient to fully resolve the spectral lines of interest. In spite of the $0.2 \mathrm{~cm}^{-1}$ line widths, we find that resolutions as broad as $8 \mathrm{~cm}^{-1}$ should be considered to maximize the SNR. This is 
an extremely important result since it indicates that incorporating a less expensive, low-resolution spectrometer in a dedicated $\mathrm{H}_{2} \mathrm{O}$ detection instrument is consistent with achieving maximal sensitivity.

There are two issues which were not discussed above that may influence the choice of resolution. First, for CLS applications, spectral resolution may influence the degree of adherence to Beer's law. This possibility is currently being investigated. Second, the results presented in this section are based upon a fixed beam aperture, which is small enough to acquire narrow resolution spectra.. However, for broad resolution spectra the aperture can be opened thereby allowing a higher instrument throughput. Maximizing the instrument throughput for each resolution further favors broad resolution measurements.

Fig. 23 shows spectra over the nearly two decades of spectral resolution that were studied. Using a resolution narrower than the $8 \mathrm{~cm}^{-1}$ optimum is appealing because it allows a clear delineation of peaks selected in the CLS routine, and it allows ready recognition of spectral interferences. Hence, the choice of resolution has a subjective component in addition to the above considerations. With these factors in mind we chose to do the bulk of our work at $1 \mathrm{~cm}^{-1}$ resolution.

\subsection{Choice of Apodization Function}

The fast Fourier transform (FFT), as it is commonly applied to FTIR spectroscopy, assumes that the single beam spectrum can be exactly represented by the sum of a discrete number of cosine functions [16]. However, due to the continuous and bounded nature of the actual spectrum, this assumption is never strictly true. The effect of such an assumption on realistic spectral data is shown in Fig. 24, where an FFT was used to produce a spectrum based upon a simulated interferogram corresponding to an isolated absorption line. It can be seen that the resulting spectrum displays side-lobes or "feet," which were not a feature of the original spectrum.

The usual method of alleviating these artifacts is to scale the interferogram values starting with unity at the center of the mirror motion and progressing to about zero at the extremes of the mirror motion This technique is known apodization, coming from the term apodal: without feet. There are many apodization functions, which differ greatly in the form and severity. An extreme form of apodization is known as the boxcar function, which is the term used to describe a function that is 1.0 within some region and 0.0 elsewhere, as illustrated in the bottom of Fig. 24. When the width of the boxcar is set to the width of the measured interferogram, the absorption spectrum contains the oscillating artifacts shown in the top of Fig. 24. Unapodized spectra are commonly said to have a boxcar apodization applied to them.

Two common apodization functions are the triangular and the Happ-Genzel functions. The triangular apodization function is unity at the center of the interferogram and decreases linearly to zero at the last point of the interferogram, as shown in the bottom of Fig. 25 . The 
effect that this apodization has on the spectrum of an isolated line is shown in the top of Fig. 25. Both the oscillations and the height of the tails are greatly reduced relative to the unapodized spectrum shown in Fig. 24. Also, the peak height of the line has been reduced to approximately $67 \%$ of the height of the peak in the unapodized spectrum. The exact value of this reduction varies with application.

The Happ-Genzel apodization function is described by [17]:

$$
h(x)=0.54+0.46 \cos \left(\pi x / x_{\max }\right)
$$

where $\mathrm{x}$ is the independent parameter of the interferogram corresponding to the position of the scanning mirror, and ranges, in a two-sided interferogram, from $-x_{\max }$ to $x_{\max }$. The form of this function and the effect that it has on an isolated-line spectrum is shown in Fig. 26.

Happ-Genzel apodization decreases the average magnitude of the tails even more than the triangular apodization, at the expense of increased oscillation of the tails around the local mean. For this application, the peak height of the line is roughly $72 \%$ of the height of the peak in the unapclized spectrum.

In practice, triangular and Happ-Genzel apodization functions yield spectra with very similar characteristics, either of which greatly reduces the istifacts evident in an unapodized spectrum. Fig. 27 compares the effects of apodization on a portion of the full spectrum for the cases discussed above. The spectra have a norainal resolution of approximately $0.96 \mathrm{~cm}^{-1}$. The bottom trace in Fig. 27 shows an ideal spectrum for comparison. It corresponds to a spectrum with infinitesimal resolution that is binned into discrete elements, $0.48 \mathrm{~cm}^{-1}$ wide.

The spectra presented in this report have Happ-Genzel apodization applied to them, unless otherwise noted. This produces a visually appealing spectrum without significantly broadening the spectra. Subjectively, apodization aides our peak selection process and other elements of human interpretation of the spectra. The effect of apodization on quantitative results using the univariate approach is discussed elsewhere [18]. The effect on CLS calculations is under study.

\subsection{Choice of Optical Sample Cell}

The White and Axiom cells have been described in Sec. 2.3. The White cell is certainly a more efficient design for trace gas analysis with its high ratio of transmission to path length. However, the White cell used in this experiment could not be heated significantly nor dried lower than about $150 \mathrm{ppb}$. Also, it proved to be a significant moisture sink and source, so that it is probably a poor choice for in-line monitoring. A White cell with considerably improved design has recently become available from Connecticut Instruments and will be tested at our facility.

With heating, the four-tube configuration of the Axiom cell could be dried to about 30 $\mathrm{ppb}$ and the two tube configuration to about $15 \mathrm{ppb}$. Hence, this cell was preferred for 
work in the lower ppb range.. Since the Axiom cell more nearly approximates plug flow, it seems more acceptable for an in-line application. However, more work is necessary to arrive at a design for the optical cell of the prototype instrument to be discussed in Section 5.

\subsection{Choice of Path Length in Optical Sample Cell}

The Axiom gas cell, which was used for many of the experiments described in this report, could be configured to lengths between 4 and $16 \mathrm{~m}$, in $4 \mathrm{~m}$ increments. To choose the length, an analysis was performed of the SNR associated with a univariate absorption measurement as a function of the length of the gas cell. The absorbance due to the $\mathrm{H}_{2} \mathrm{O}$ in the gas cell, $A_{c}$, is the absorbance of the gas cell plus instrument, $A_{m}$, minus the absorbance of the instrument (i.e., $\mathrm{H}_{2} \mathrm{O}$ in the optical path of the spectrometer), $\mathrm{A}_{0}$. In terms of the corresponding single beam values, the absorbance of the $\mathrm{H}_{2} \mathrm{O}$ in the gas cell is given by,

$$
A_{c}=\log _{10}\left[\left(S_{o p} S_{m b}\right) /\left(S_{o b} S_{m p}\right)\right] \text {, }
$$

where

$$
\begin{aligned}
& S_{\mathrm{mp}}=\text { single beam peak value (gas sample }+ \text { instrument), } \\
& \mathbf{S}_{\mathrm{mb}}=\text { single beam baseline value (gas sample }+ \text { instrument), } \\
& S_{\mathrm{op}}=\text { single beam peak value (instrument only), } \\
& S_{\mathrm{ob}}=\text { single beam baseline value (instrument only). }
\end{aligned}
$$

In terms of the real experimental system, the single beam values designated as "gas sample + instrument" correspond to spectra measured with the InSb detector, while the values designated as "instrument only" correspond to those spectra measured with the TGS detector.

A propagation-of-errors analysis results in the following expression for the variance of the absorbance estimate due to the gas in the cell:

$$
\sigma_{\mathrm{Ac}}{ }^{2}=\log _{10}{ }^{2}[e]\left(\left(\sigma_{\mathrm{TOS}}{ }^{2} / \mathrm{S}_{\mathrm{op}}{ }^{2}\right)+\left(\sigma_{\mathrm{lasb}}{ }^{2} / \mathrm{S}_{\mathrm{mpp}}{ }^{2}\right)+\left(\mathrm{a}_{\mathrm{p}} \mathrm{l}_{\mathrm{l}}\right)^{2} \sigma_{\mathrm{bkg}}{ }^{2}\right\},
$$

where,

$\sigma_{\mathrm{TOs}}{ }^{2}=$ the variance of the noise in the signal from the TGS detector,

$\sigma_{\mathrm{less}}{ }^{2}=$ the variance of the noise in the signal from the InSb detector,

$a_{p}=$ absorptivity at the peak of the absorption line,

$\mathrm{l}_{1}=$ optical path length within the spectrometer,

$\sigma_{b k g}{ }^{2}=$ variance of the spectrometer $\mathrm{H}_{2} \mathrm{O}$ concentration between the time that the TGS and InSb measurements are made (typically 10 minutes),

and it is assumed that the detector noise is independent of the signal. The first term in the braces, $\sigma_{\mathrm{Tos}}{ }^{2} / \mathrm{S}_{\mathrm{op}}{ }^{2}$, is approximately equal to half the mean-square deviation observed along the $100 \%$ line calculated using the TGS spectra, and was experimentally found to be 
$2.47 \times 10^{-8}$. Similarly, the term, $\sigma_{\mathrm{lssb}}{ }^{2} / \mathrm{S}_{\mathrm{mp}}{ }^{2}$, was experimentally determined to be $\left(8.65 \times 10^{-11} / \mathrm{T}^{2 \mathrm{Lol} / 0}\right)$, where $\mathrm{L}_{\mathrm{c}}$ is length of the gas cell, $\mathrm{T}$ is the intensity transmittance of the gas cell per length $L_{0}$, and the assumption is made that the absorbance due to $\mathrm{H}_{2} \mathrm{O}$ in the gas cell is negligible compared to the transmission of the cell. The transmission of the Axiom gas cell in our possession was experimentally determined be 0.70 per 2 -meter section. This compares to a value of 0.67 inferred from the company literature. The product $a_{1} l_{1}$ is equal to $1.79 \times 10^{-6} \mathrm{AU} / \mathrm{ppb}$ for a typical $\mathrm{H}_{2} \mathrm{O}$ absorption line (i.e., at $3744.6 \mathrm{~cm}^{-1}$ ). Finally, the variance of the background $\mathrm{H}_{2} \mathrm{O}$ concentration, $\sigma_{\text {bkg }}{ }^{2}$, was estimated to be $(1.77 \mathrm{ppb})^{2}$ for a ten-minute interval between TGS and InSb measurements made when the spectrometer was well conditioned (i.e., purged for two weeks or longer).

The SNR of the univariate estimate of the absorbance due to the $\mathrm{H}_{2} \mathrm{O}$ in the gas cell, $\mathrm{SNR}_{\mathrm{Ao}}$, can be found by combining Eqs. 22,23 , and the preceding values:

$$
\operatorname{SNR}_{A c}=I_{c} /\left[2.471 \times 10^{-4}+\left(8.65 \times 10^{-11} / 0.7^{10}\right)\right]^{\mathrm{h}} .
$$

A plot of this parameter as a function of the length of the gas cell is given in Fig. 28. The peak of the SNR occurs at a gas cell length of $14.5 \mathrm{~m}$. The peak is broad and its position is critically dependent on the ratio of $\sigma_{\text {Tos }}$ to $\sigma_{\text {Inst }}$. In the simplest case when there is a single detector and $\sigma_{b k g}{ }^{2}=0$, the length calculated for maximal SNR is $6.2 \mathrm{~m}$. For the same case, the optimal path length for the White cell (assuming $T=0.97$ and $L=0.55 \mathrm{~m}$ ) is $20 \mathrm{~m}$.

The mathematical analysis was merged with more subjective considerations to reach a final decision. Conditioning the Axiom cell by heating and purging proved to be lengthy procedure that is quite critical to achieving reproducible results below about $200 \mathrm{ppb}$. Of course, a shorter cell with its smaller surface area makes this task easier. Therefore, in most of the experiments, we chose to configure the Axiom cell for an $8 \mathrm{~m}$ path length (i.e., four tubes).

3.7 Summary of Standard Experimental Conditions

1. Spectral resolution $-1.0 \mathrm{~cm}^{-1}$.

2. Number of Scans, TGS - 180 (10 min).

3. Number of Scans, InSb - $620(10 \mathrm{~min})$.

4. Source - glowbar.

5. Beamsplitter - KBr.

6. Optical cell windows - $\mathrm{KBr}$.

6. Spectral region -3600 to $3910 \mathrm{~cm}^{-1}$.

7. CLS regions - see Fig. 6, with band by band analysis.

8. CLS factors - one.

9. Apodization - Happ-Genzel.

10. Zero-filling - two times, yielding one point per $0.25 \mathrm{~cm}^{-1}$.

11. Optical filters - none.

12. Temperature $-23^{\circ} \mathrm{C}, \pm 2^{\circ}$. 
13. Pressure - Atmospheric $=0.822 \pm 0.013 \mathrm{~atm}$, Gauge $=0.544 \pm 0.014 \mathrm{~atm}$, Total $=1.37 \pm 0.02 \mathrm{~atm}$.

14. Flow rate of sample $=1$ to $10 \mathrm{sim}$ as dictated by desired moisture level.

15. Axiom cell path length $=8 \mathrm{~m}$.

16. White cell path length $=22 \mathrm{~m}$. 


\subsection{Calibration and Detection Limits}

Figs. 29 and 30 plot the $\mathrm{H}_{2} \mathrm{O}$ concentration in $\mathrm{N}_{2}$ determined spectroscopically versus the concentration determined by the reference method, the Aquamatic Plus. Fig 29 pertains to the White cell, while Fig. 30 pertains to the Axiom cell. The inverse of the slope of the bestfit line gives a calibration for the arbitrary CLS units. For Fig. 29 the calibration is $44.1 \mathrm{ppb}$ per CLS unit (standard error $=1.1 \mathrm{ppb}$ ), while for Fig. 30 the calibration is $106.3 \mathrm{ppb}$ per CLS unit (standard error $=7.1 \mathrm{ppb}$ ). In comparing the two calibration results, a correction is necessary for the path length difference between the cells. Adjusting the White cell results to an $8 \mathrm{~m}$ path, yields a calibration of $121.2 \mathrm{ppb}$ per CLS unit (standard error $=2.9 \mathrm{ppb}$ ). Although reasonably close, the two calibration results may differ partly because of systematic errors. For example, the cell path lengths may be in error by a few percent. Also, the sensitivity of the Aquamatic Plus may be slightly influenced by whether it has seen mostly dry or mostly wet environments in the few weeks before an experiment.

The non-zero intercepts seen in Figs. 29 and 30 are unexplained. However, we believe that they do not have a primary affect on the accuracy of the calibration that is derived from the slope.

From the calibration discussed above it is possible to derive an absorptivity for each $\mathrm{H}_{2} \mathrm{O}$ band in the spectrum. Fig. 31 compares the absorptivities found in this work (using 106 ppb per CLS unit) with that of the HITRAN data base. The data associated with the twelve most prominent peaks from Fig. 6 are plotted. The best-fit line, while going essentially through the origin, has a slope of 0.77 . This indicates that the absorptivities determined in this work are typically $77 \%$ of those derived from HITRAN. Within HITRAN, the precision of the cross-section values for these $\mathrm{H}_{2} \mathrm{O}$ bands is listed as undetermined, indicating that the original experiments were not done with exact quantification in mind. Therefore, we consider the discrepancy in the absorptivities to be acceptable. Comparing absorptivities for water vapor bands between laboratories is a very difficult proposition. Besides the obvious problem of duplicating the reference $\mathrm{H}_{2} \mathrm{O}$ concentration, duplicating the spectrometer conditions can be a significant source of error. Especially in gas phase work, the intensity at an absorption peak is a very sensitive function of resolution and apodization. Nominally identical spectrometer conditions may not be truly identical, so that errors of 5 to $10 \%$ can easily occur.

Fig. 32 presents the data of Fig. 29 as a cross-validated calibration plot. To create Fig. 32, a series of plots like Fig. 29 were made with a single data point removed before calculating the best fit. The series, excluding each data point in turn, is used to predict the concentrations of the excluded points as if they were unknown. Finally, in Fig. 32 the predicted values of the excluded points are plotted verses their reference values. For perfect prediction, the data should lie on the solid $45^{\circ}$ line with zero intercept. The standard error of prediction (SEP), defined as the RMS deviation from the $45^{\circ}$ line, is a measure of the precision of the determination. For the high range of $\mathrm{H}_{2} \mathrm{O}$ concentrations, using the White cell, the SEP is $49 \mathrm{ppb}$. For the low range of $\mathrm{H}_{2} \mathrm{O}$ concentrations, using the Axiom cell, the cross-validated calibration plot in Fig. 33 indicates an SEP of $18 \mathrm{ppb}$. Individual 
contributions to the SEP are discussed below. It will be shown that the SEP's are dominated by the precision of the reference method and cannot be used to infer the detection limits of the FTIR instrument.

As seen in Fig. 7, the concentration of $\mathrm{H}_{2} \mathrm{O}$ is determined by two separate measurements. First, the instrument background plus sample (InSb), and second, the instrument background alone (TGS). The precision of the InSb measurement is shown in Fig. 34 , where 54 repeated measurements were made on a stable sample of $\mathrm{H}_{2} \mathrm{O}$ in $\mathrm{N}_{2}$ over a ninehour period. Since a small amount of drift (about $10 \mathrm{ppb}$, in this case) is unavoidable, the SEE is reported as a measure of the precision. The drift is modeled by a second order polynomial. Similarly, Fig. 35 shows the precision of the TGS measurements. The SEE's are InSb $=3.3 \mathrm{ppb}$ and TGS $=9.1 \mathrm{ppb}$, where the path length is set at $8 \mathrm{~m}$.

The precision for the determination of $\mathrm{H}_{2} \mathrm{O}$ in $\mathrm{HCl}$ is found to be somewhat poorer as a result of interfering species. Fig. 36 is a spectrum of about $10 \mathrm{ppm}$ of $\mathrm{H}_{2} \mathrm{O}$ in pure $\mathrm{HCl}$. The individual rotation-vibrational lines of $\mathrm{HCl}$ are so strongly absorbing that the FTIR spectrometer merges them into an envelope peaking at about $3000 \mathrm{~cm}^{-1}$. The $\mathrm{H}_{2} \mathrm{O}$ spectrum can readily be seen in the region 3600 to $3900 \mathrm{~cm}^{-1}$. Fig. 37 shows a narrower spectral region with a much lower concentration of $\mathrm{H}_{2} \mathrm{O}$ in $\mathrm{HCl}$ (about $200 \mathrm{ppb}$ from the cell and the equivalent of about $300 \mathrm{ppb}$ from the instrument background). Severe $\mathrm{CO}_{2}$ interference is evident in the Fig. 37, while $\mathrm{CH}_{4}$ may be contributing what appears to be noise in the 3800 to $3900 \mathrm{~cm}^{-1}$ spectral region. The spectra of these two gases are presented in Fig. 40 . Using the cross-section data in HITRAN, it was verified that the semiconductor grade $\mathrm{HCl}$ met its specification of $<10 \mathrm{ppm} \mathrm{CO}_{2}$ and $<2 \mathrm{ppm} \mathrm{CH}_{4}$. When performing the CLS calculation on spectra like Fig. 37, only the five regions between 3800 and $3900 \mathrm{~cm}^{-1}$ were chosen (see Fig. 6). The precision obtained by repeated measurements is indicated by Fig. 38, where the SEE is $8.4 \mathrm{ppb}$ at an $8 \mathrm{~m}$ path length. This compares to an SEE of $3.3 \mathrm{ppb}$ in $\mathrm{N}_{2}$ under identical conditions.

From the errors determined experimentally ( $18 \mathrm{ppb}$ for the low range calibration, 3.3 $\mathrm{ppb}$ for InSb, and $9.1 \mathrm{ppb}$ for TGS) the error of the reference method is calculated as follows:

$$
\sigma_{\text {ref }}=\left[18 \mathrm{ppb}^{2}-3.3 \mathrm{ppb}^{2}-9.1 \mathrm{ppb}^{2}\right]^{\mathrm{h}}=15.2 \mathrm{ppb}
$$

The $15.2 \mathrm{ppb}$ error attributed to the reference is consistent with the specified performance of the Aquamatic Plus. Following an analogous calculation for the high range calibration (Fig. 33), the error attributed to the reference method is much higher at $48.0 \mathrm{ppb}$. While the error of the Aquamatic Plus must be near $15 \mathrm{ppb}$ for this case also, there is the added problem of attaining adequate equilibrium with the White cell. Errors due to lack of achieving equilibrium are indistingıishable from errors due to the Aquamatic Plus and may explain the higher SEP.

Considering only the errors from the FTIR measurements, it is evident that detection limits for the current instrument are: $9.7 \mathrm{ppb}(3.3 \mathrm{ppb}, 9.1 \mathrm{ppb})$ for $\mathrm{H}_{2} \mathrm{O}$ in $\mathrm{N}_{2}$, and $12.4 \mathrm{ppb}$ 
(8.4 ppb, $9.1 \mathrm{ppb}$ ) for $\mathrm{H}_{2} \mathrm{O}$ in $\mathrm{HCl}$. By substituting an InSb detector for the low performance TGS detector the background measurement could be made with a precision of $0.8 \mathrm{ppb}=(3.3$ $\mathrm{ppb}) \times\left(0.7^{4}\right)$, where the additional factor accounts for the increased light available when the beam does not pass through the cell. The improved detection limits would be: $3.4 \mathrm{ppb}$ (3.3 $\mathrm{ppb}, 0.8 \mathrm{ppb}$ ) for $\mathrm{H}_{2} \mathrm{O}$ in $\mathrm{N}_{2}$, and $8.4 \mathrm{ppb}(8.4 \mathrm{ppb}, 0.8 \mathrm{ppb})$ for $\mathrm{H}_{2} \mathrm{O}$ in $\mathrm{HCl}$. Other improvements are discussed in the next section. Table 2 summarizes the detection limits for the present instrument.

\begin{tabular}{|l|c|c|}
\hline & \multicolumn{2}{|c|}{$1 \sigma$ Detection Limits (ppb) } \\
\hline & InSb + TGS & $2 \times$ InSb \\
\hline $\mathrm{H}_{2} \mathrm{O}$ in $\mathrm{N}_{2}$ & 9.7 & 3.4 \\
\hline $\mathrm{H}_{2} \mathrm{O}$ in $\mathrm{HCl}$ & 12.4 & 8.4 \\
\hline
\end{tabular}

Table 2 - Detection limits of the present instrument operated with a path length of $8 \mathrm{~m}$. 


\subsection{Design Improvements}

\subsection{Description of a Mark II Prototype System}

The FTIR spectrometer and related equipment described above were chosen to provide a versatile and efficient means of demonstrating the feasibility of trace $\mathrm{H}_{2} \mathrm{O}$ detection by infrared spectroscopy. However, improvements in cost and performance can be realized by optimizing the design of a dedicated instrument.

The current mechanism for switching between sample and background measurements is slow and requires that all the scans for one measurement be completed before beginning the other. For best results, the concentration of moisture in the instrument must be constant for the total measurement time of 20 minutes. A one- to two-week purge of the present instrument is needed to give acceptable performance.

The new spectrometer will use a single InSb detector with two nearly identical beam paths, such as in Fig. 39. The motion of the bypass mirror is synchronized with the spectrometer scanning mirror to alternately collect the sample and background interferograms, at about one scan per second. This minimizes the effect of the background drift. More promising methods for making simultaneous measurements of the sample and background absorbances are currently under consideration, but will not be discussed at this time. We feel that a dual beam instrument can be operated with a normal purge level and still maintain detection limits similar to those reported herein. Of course, minimizing the beam path external to the cell is another design feature that will the ease purge requirements.

Unlike the current spectrometer, which is able to collect data with a nominal resolution as narrow as $0.08 \mathrm{~cm}^{-1}$, a spectrometer dedicated to trace $\mathrm{H}_{2} \mathrm{O}$ determination requires a resolution of only $2 \mathrm{~cm}^{-1}$. This permits the use of a compact and inexpensive scanning mechanism. Examples are the sliding quartz wedges used by Analect, and the wishbone scanner with cube corner reflectors used by Bomem. Also, broad resolution allows a large aperture in the spectrometer, thereby increasing the light incident on the detector.

Significant improvement in the SNR can be realized with the addition of an optical bandpass filter to limit the light incident on the detector to only the frequencies of interest. The area under the full InSb single beam spectrum is approximately 14 times greater than that between 3600 and $3900 \mathrm{~cm}^{-1}$. Therefore, with an appropriate optical bandpass filter, the intensity from 3600 to $3900 \mathrm{~cm}^{-1}$ can be increased approximately 14 times without saturating the detector. Since the instrument noise is dominated by signal independent detector noise, a 14-fold increase in light intensity will produce the same increase in the SNR. This is particularly critical when using an InSb detector. Recall from Section 3.1 that, typically, the intensity available from a glowbar source must be attenuated by a factor of about 30 to avoid saturating an InSb detector. 
For the current work, the electronic bandpass filters in the spectrometer were set to collect spectra from 0 to $7900 \mathrm{~cm}^{-1}$. Since we are interested in only a small portion of the spectrum, the electronic bandpass should be trimmed as close to the relevant frequency range as possible. Assuming the detector noise has a white spectral content, and the electronic filters are ideal, one would expect proper filtering to reduce the noise by a factor of 5 below current levels $\left(\sigma_{\text {ideal }} / \sigma_{\text {curreat }}=[300 / 7900]^{1 / 2}\right)$. However, the theoretical improvement expected with electronic filtering may be only partly realized, since the roll-off will distort the spectrum if set too close to the frequencies of interest.

The current implementation of the CLS algorithm considers only a single component, $\mathrm{H}_{2} \mathrm{O}$, in addition to a linear baseline. However, semiconductor grade $\mathrm{HCl}$ can have a significant amount of other gases that absorb in the 3600 to $3900 \mathrm{~cm}^{-1}$ region. The $\mathrm{HCl}$ used in these experiments has a specified limit of $10 \mathrm{ppm}$ for $\mathrm{CO}_{2}$, and $2 \mathrm{ppm}$ for $\mathrm{CH}_{4}$. Fig. 40 shows the absorption spectra for $\mathrm{H}_{2} \mathrm{O}, \mathrm{CO}_{2}$, and $\mathrm{CH}_{4}$ at concentrations of $1 \mathrm{ppb}, 1 \mathrm{ppm}$, and 1 ppm, respectively. Both $\mathrm{CO}_{2}$ and $\mathrm{CH}_{4}$ have appreciable overlap with $\mathrm{H}_{2} \mathrm{O}$. Because of the $\mathrm{CO}_{2}$ interference, only the region from 3788 to $3910 \mathrm{~cm}^{-1}$ was used for a single-component CLS determination of $\mathrm{H}_{2} \mathrm{O}$ reported in Section 4. A three-component CLS analysis should improve the $\mathrm{H}_{2} \mathrm{O}$ estimate, and will provide information on $\mathrm{CO}_{2}$ and $\mathrm{CH}_{4}$ impurity levels.

At present, we are favoring the incorporation of an Axiom type cell into the redesigned instrument. Ruggedness, ease of fabrication, ease of optical alignment, and superior conditioning characteristics are principal considerations. However, it is quite possible that a properly designed multipass gas cell can provide a better SNR. The problem, to minimize the volume and surface area for a given path length, is still under consideration. Regardless of cell type, it must be possible to pressurize, evacuate, and heat the cell.

Assuming the use of an Axiom gas cell, it is important to consider the optimal path length. For a single detector in a true dual beam instrument, Eq. 23 predicts an optimum path length of $6.2 \mathrm{~m}$. Fig. 41 shows the SNR verses the path length for such an instrument, assuming a univariate estimator. Reproduced from Fig. 28 is the analogous curve for the present instrument. The curves have been scaled so that the peak SNR of the current system is unity. By choosing the optimum path length of $6.2 \mathrm{~m}$, the redesigned system is expected to achieve a factor of 4.2 improvement in SNR over the current system.

\subsection{Estimate of Mark II Performance}

Table 3, on the next page, summarizes the suggested improvements for the Mark II instrument. The first three improvements do not change the detection limit but provide other important advantages as listed in the comments in Table 3. The use of an optical filter to isolate the $3600-3900 \mathrm{~cm}^{-1}$ region provides a factor of 14 improvement in the SNR, while an ideal electronic filter may yield an additional factor of 5 . Using multiple components in the CLS calculation to properly account for known spectral interferences allows the use of the whole spectrum, or about twice the number of points, giving an improvement of about $\sqrt{2}$. If the path length of the gas cell in the new system were $70 \mathrm{~cm}$, instead of the $6.2 \mathrm{~m}$ optimum, 
the cell would provide about the same SNR as achieved with the $8 \mathrm{~m}$ path in the current system (see Fig. 41). The shorter path length is recommended since the cell will be easier to install, easier to condition, and have a faster response time than a longer cell. With these enhancements to the SNR it possible to shorten the collection time substantially and still maintain a detection limit superior to the present instrument. A collection time of one minute is suggested.

By dividing the $1 \sigma$ detection limit in Table $2(8.4 \mathrm{ppb})$ by the expected improvements in Table 3 (31), we arrive at a projected detection limit of $0.27 \mathrm{ppb}$. While this, no doubt, is optimistic, a detection limit for the Mark II instrument of about 1 ppb seems quite conservative. Regardless of the sensor technology employed, the accuracy of low ppb measurements is degraded by surface adsorption effects within the gas delivery system being monitored. Because of this, detection limits lower than the $1 \mathrm{ppb}$ expected for the second generation instrument may have little practical value.

Our laboratory instrument can be duplicated in its essential parts for about $\$ 90 \mathrm{~K}$. Given the cost savings anticipated by using a small optical cell and a low resolution spectrometer, it is quite realistic to project a cost of $\$ 50 \mathrm{~K}$ for a dedicated instrument. We are also designing an accessory for existing FTIR spectrometers that may be marketed for as little as \$10K.

\begin{tabular}{|l|c|l|}
\hline \multicolumn{1}{|c|}{ Improvement } & Change & \multicolumn{1}{c|}{ Comments } \\
\hline Dual Beam & $1 \times$ & allows poorer purge \\
\hline Low resolution & $1 \times$ & reduces cost \\
\hline Compact, well sealed & $1 \times$ & decreases purge time : \\
\hline Optical Bandpass & $14 \times$ & \\
\hline Electronic Bandpass & $5 \times$ & \\
\hline Multi-component CLS & $\sqrt{2 \times}$ & detect other species \\
\hline Short Path (70 cm) & $1 \times$ & faster equilibration \\
\hline Short scan (1 min) & $1 / \sqrt{ } 10 \times$ & effectively real time \\
\hline TOTAL CHANGE & $31 \times$ & \\
\hline
\end{tabular}

Table 3 - Summary of changes in the detection limits versus those of the present instrument. 


\section{References}

1. J.J. McAndrew and Daniel Boucheron, "Moisture Analysis in Process Gas Streams," Solid State Technology, p. 55, Feb. 1992.

2. K. Carr-Brion, "Moisture Sensors in Process Control," Elsevier, New York, 1986.

3. B.J. Streusand and R.K. Rowe, "A Review of Technology Applicable to the Determination of Trace Water Vapor in $\mathrm{HCl}$ by Absorption Spectroscopy," available through the CFM and SEMATECH, May 1993.

4. D. Pivonka, "The Infrared Spectroscopic Determination of Moisture in $\mathrm{HCl}$ for the Characterization of $\mathrm{HCl}$ Gas Drying Resin Performance," Appl. Spect., 45, 597, 1991.

5. Miyazaki, Y. Ogawara, and T. Kimura, "Analysis of Trace Moisture in $\mathrm{HCl}$ by Gas-Phase FTIR," Bull. Chem. Soc. Jpn., 66, 969, 1993.

6. K.D. Cleaver, J.W. Epton, A. Tinkler, P. Clarke, and R. Hogle, "The Application of Fourier Transform Infrared Spectroscopy to the Determination of Impurities in Semiconductor Grade Special Gas Products," private communication.

7. W.I. Bailey, T.M. Booth, D.L. Grieble, and P.B. Henderson, "Moisture Analysis in Corrosive Gases," private communication (to be published).

8. D.M. Haaland and D. Melgaard, the PLSSNL software is available from the authors at Sandia National Laboratories.

9. D.M. Haaland and R.G. Easterling, "Improved Sensitivity of Infrared Spectroscopy by the Application of Least Squares Methods," Appl. Spect., 34, 539, 1980. ( Note that there are some typographical errors in the mathematical expressions in this paper.)

10. Staff report, "Optical Detectors Across the Spectrum," Lasers and Optronics, p. 19, Sep. 1992.

11. M.P. Fuller, G.L. Ritter, and C.S. Draper, "Partial Least-Squares Quantitative Analysis of Infrared Spectroscopic Data. Parts I: Algorithm Implementation," A ppl. Spect., 42, 217. 1988.

12. D.M. Haaland, R.G. Easterling, and D.A. Vopicka, "Multivariate Least-Squares Methods Applied to the Quantitative Spectral Analysis of Multicomponent Samples," Appl. Spect., 39, 73, 1985.

13. E.V. Thomas and D.M. Haaland, "Comparison of Multivariate Calibration Methods for Quantitative Spectral Analysis," Anal. Chem., 62, 1091, 1990. 
14. L.S. Rothman, R.R. Gamache, R.H. Tipping, C. P. Rinsland, M.A.H. Smith, D.C. Benner, V.M. Devi, J.M. Flaud, C. Camy-Peyret, A. Perrin, A. Goldman, S.T. Massie, L.R. Brown, and R.A. Toth, "The HITRAN Molecular Database: Editions of 1991 and 1992," J. Quant. Spect. Radiat. Transfer, 48, 469, 1992.

15. P.R. Griffiths and J.A. de Haseth, Fourier Transform Infrared Spectroscopy, John Wiley \& Sons, New York, 1986, p. 254.

16. J.B. Bell, Introductory Fourier Transform Spectroscopy, Academic Press, New York, 1972, p. 33.

17. Griffiths, p. 20.

18. Griffiths, p. 15. 
Figures

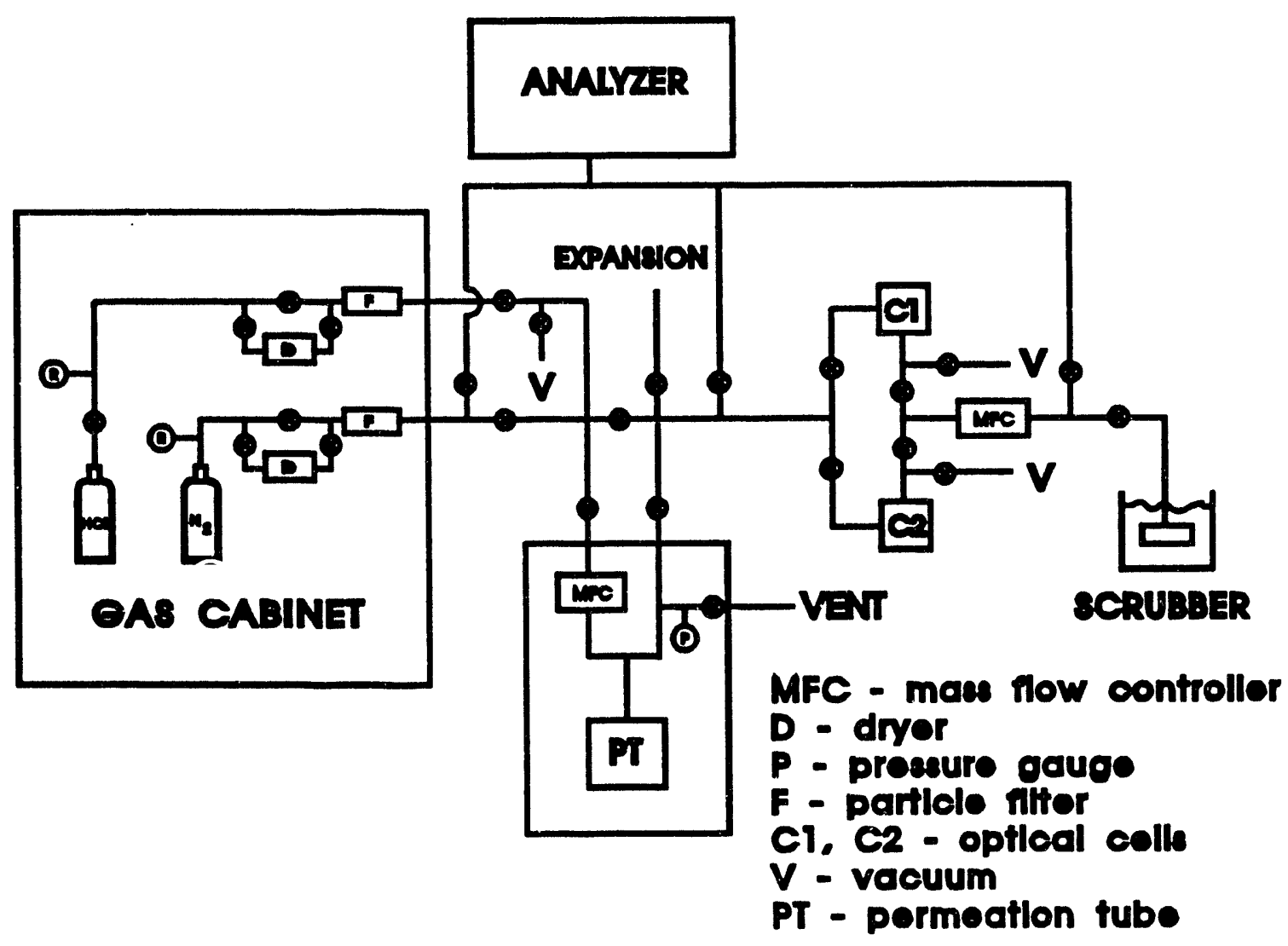

Fig. 1. Schematic of the gas handling system designed to operate with either $\mathrm{N}_{2}$ or $\mathrm{HCl}$. 


\section{Spectrometer}

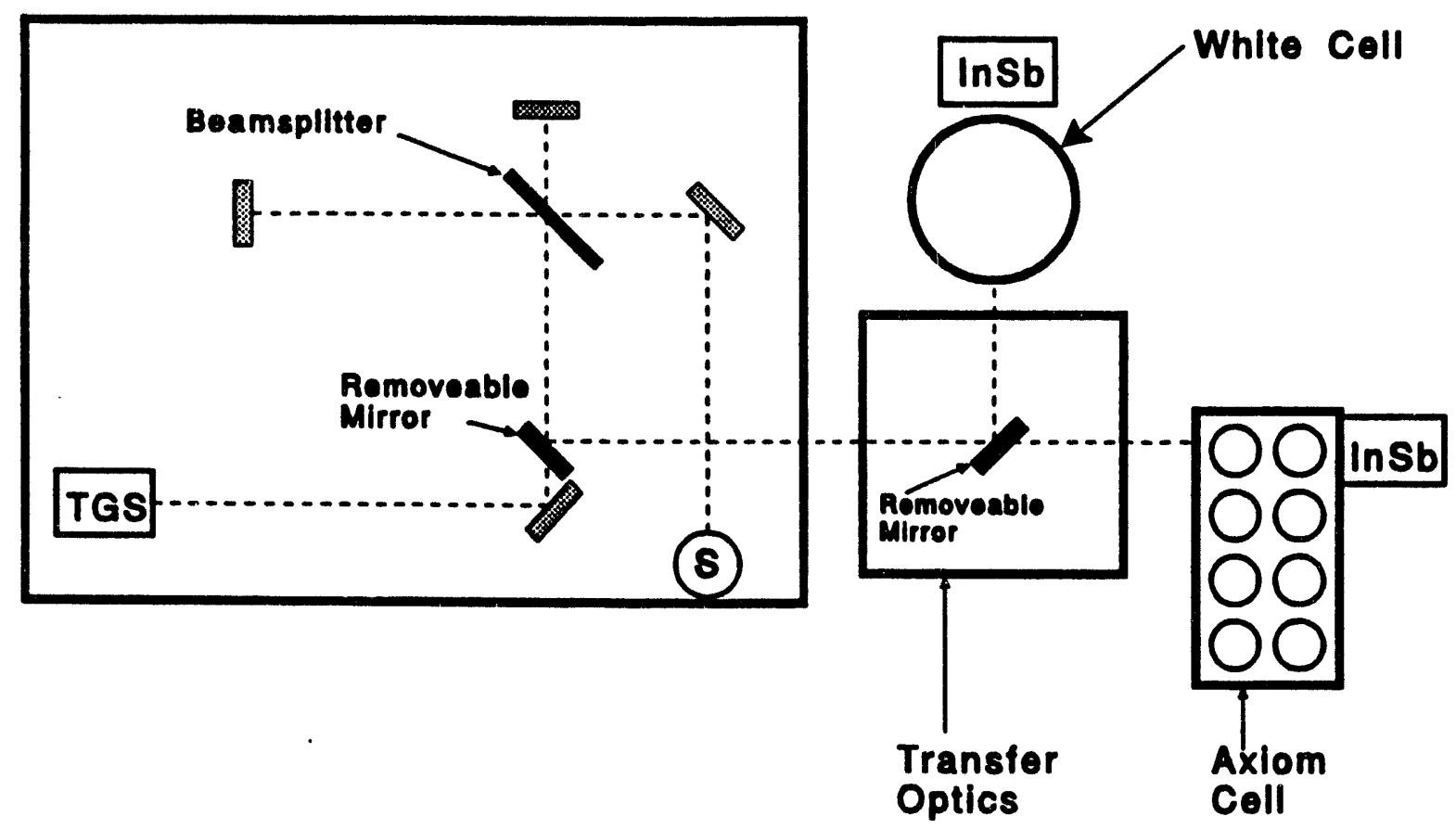

Fig. 2. Optical layout showing the infrared optical paths through the analysis system. $S$ is the glowbar source, and InSb and TGS indicate the detector positions. 


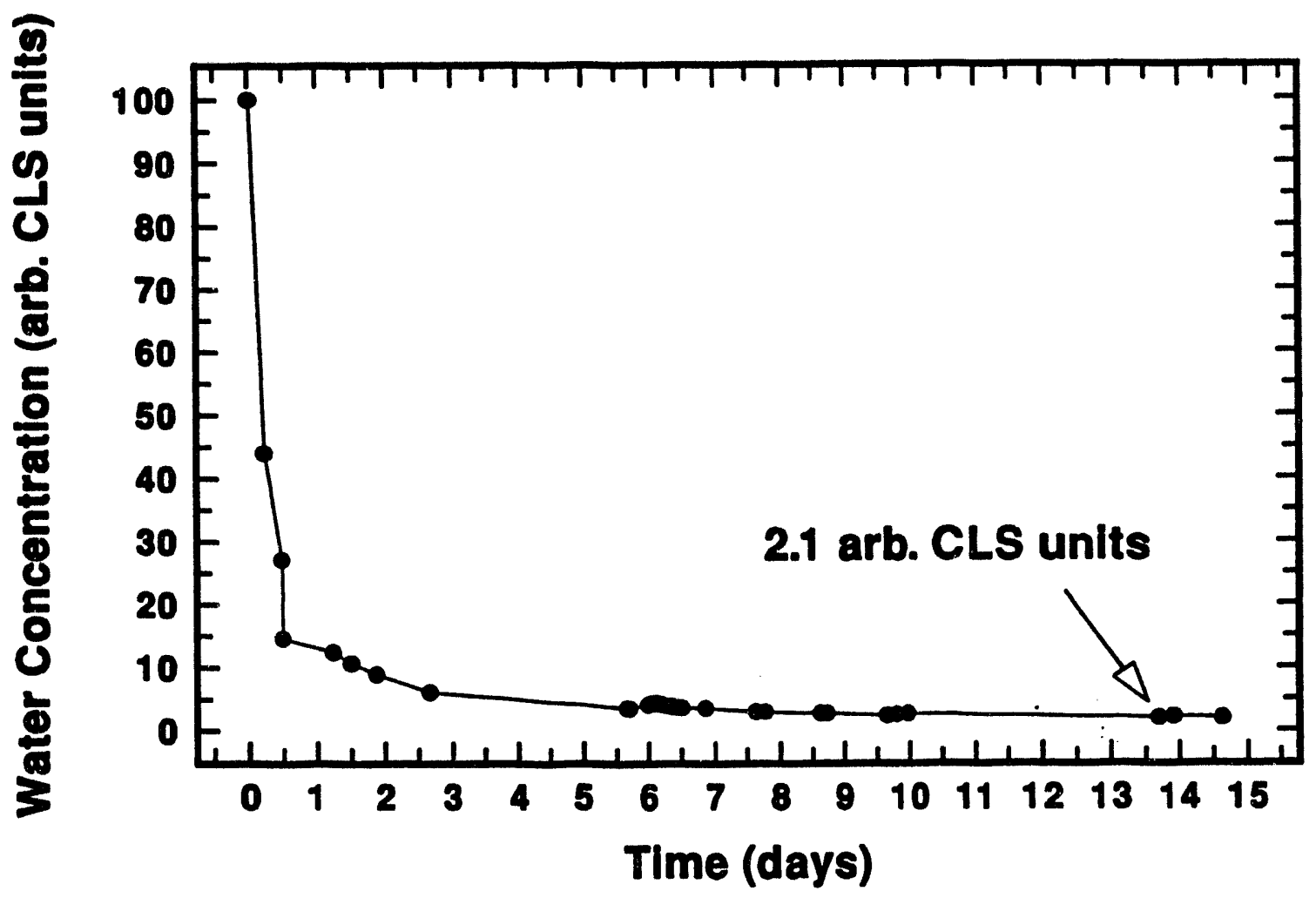

Fig. 3. Typical drydown characteristics of the modified Nicolet 800 spectrometer as measured by the TGS detector. See the text for an explanation of the arbitrary CLS units. 


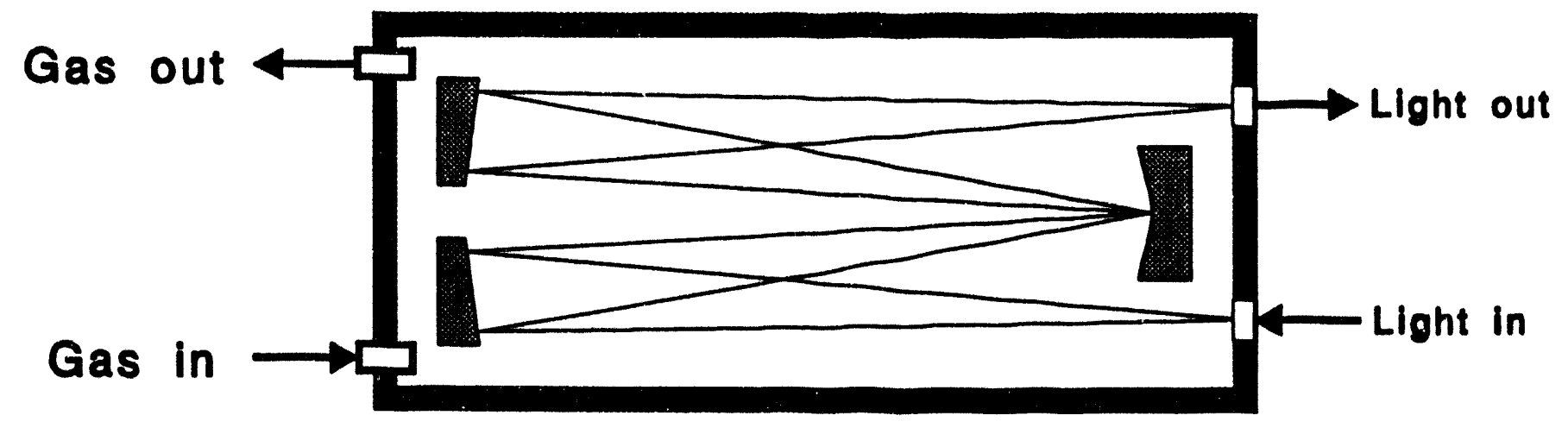

Fig. 4. A multi-pass White gas cell. The cell used in this work has a base pathlength of approximately $55 \mathrm{~cm}$. The light make 40 passes through the cell resulting in a total optical path of approximately $22 \mathrm{~m}$. Only four passes are shown for simplicity. 


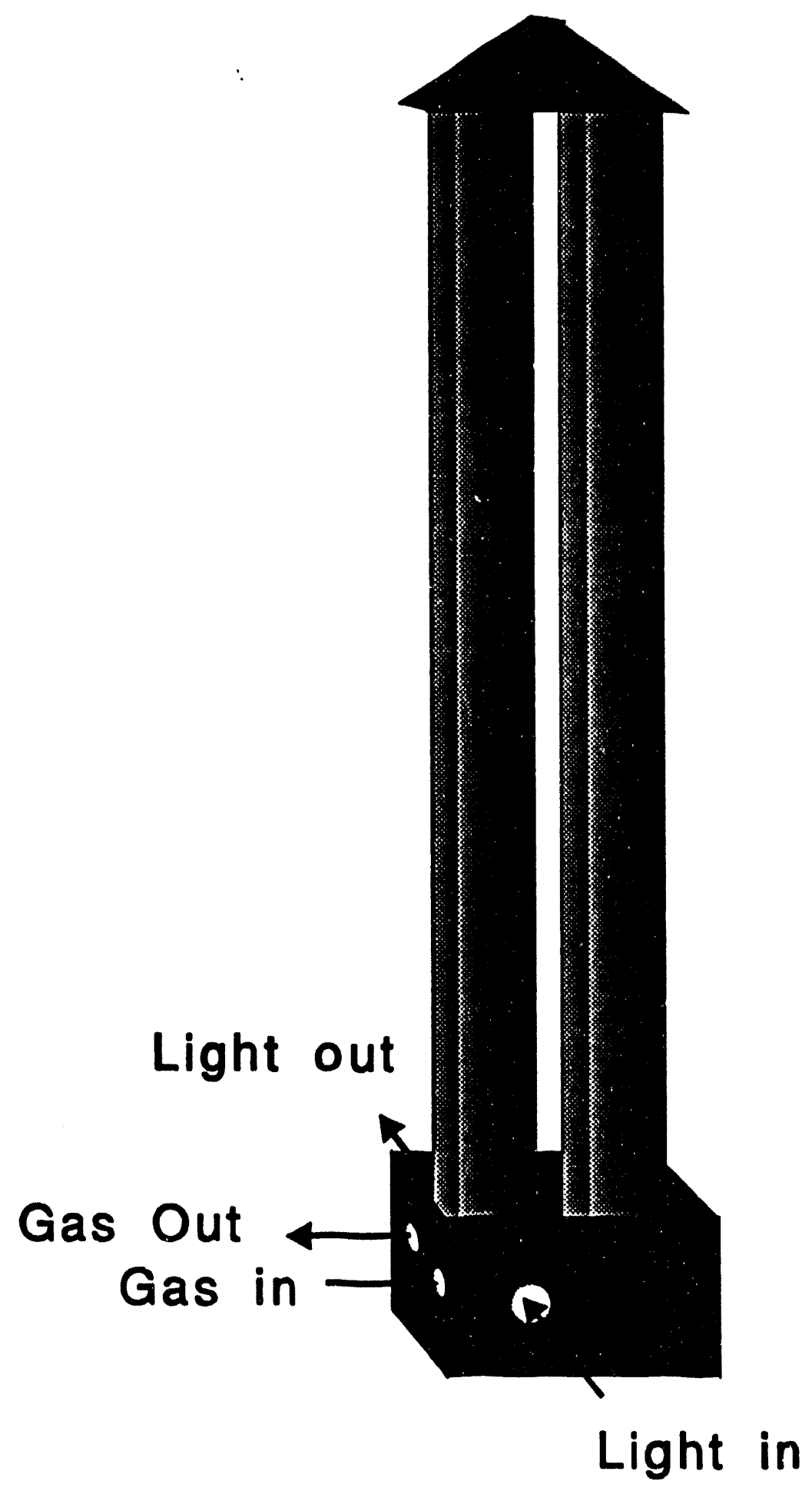

Fig. 5. A single-pass, folded Axiom gas cell. The cell is made of $2 \mathrm{~m}$ tubes (four of eight are shown) connected by gas- and light-tight endcaps that guide both the gas and light through the system. 


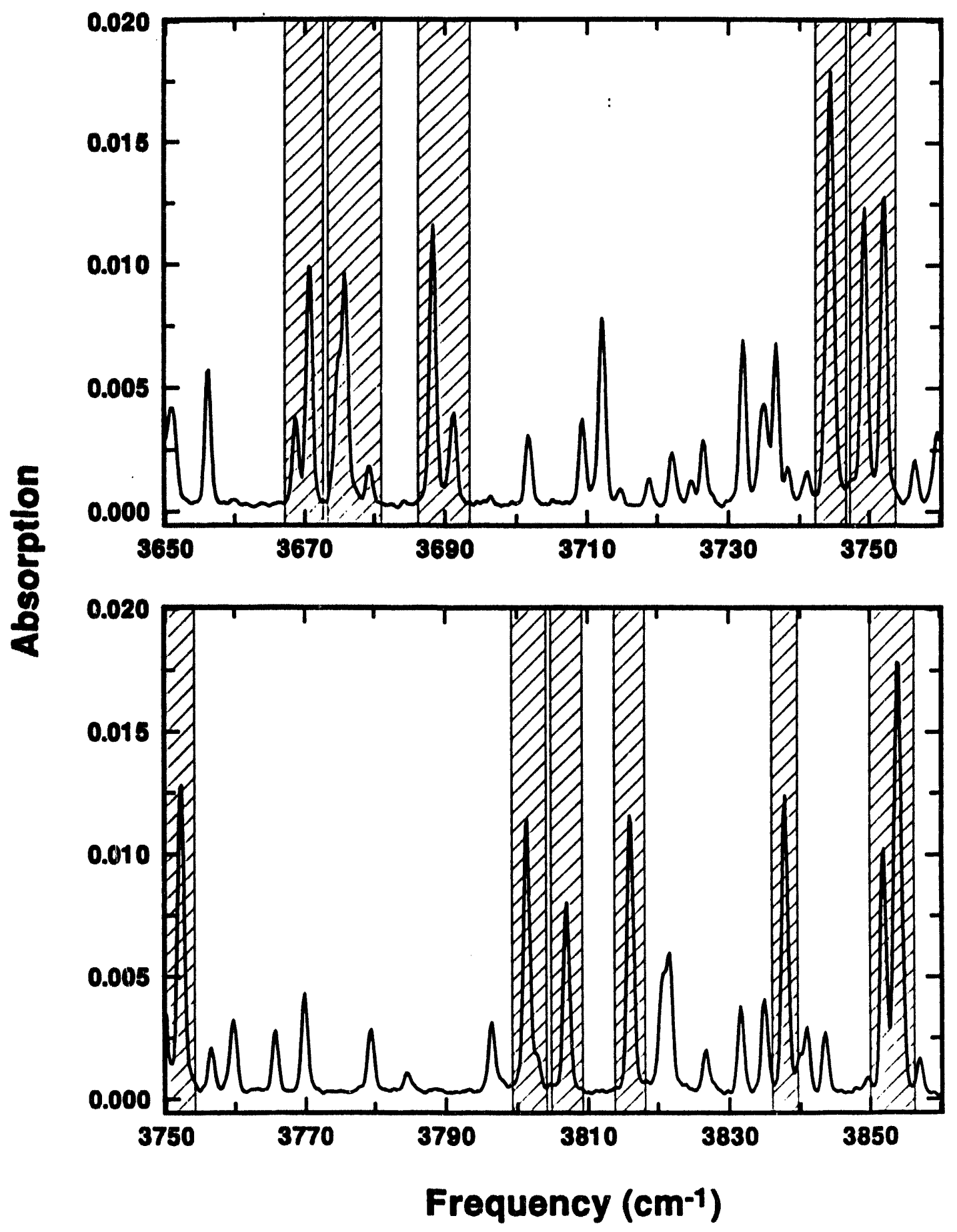

Fig. 6. Water vapor absorption spectrum $\left(1 \mathrm{~cm}^{-1}\right.$ resolution, $\left.1.37 \mathrm{~atm} ., 296 \mathrm{~K}\right)$ with superimposed cross-hatching indicating the 10 regions used for most of the CLS calculations described in this report. 


\section{Bkg.+Sample Water (InSb)}

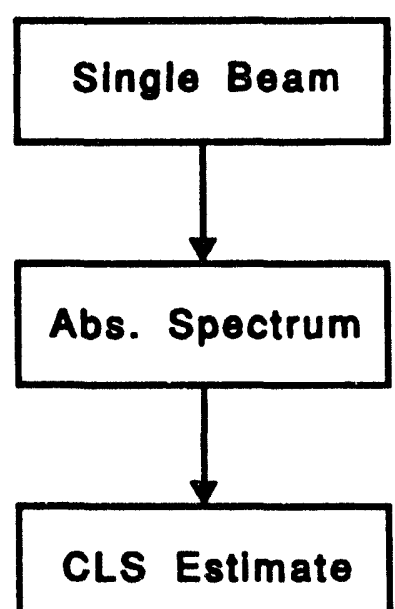

Bkg. Water

(TGS)

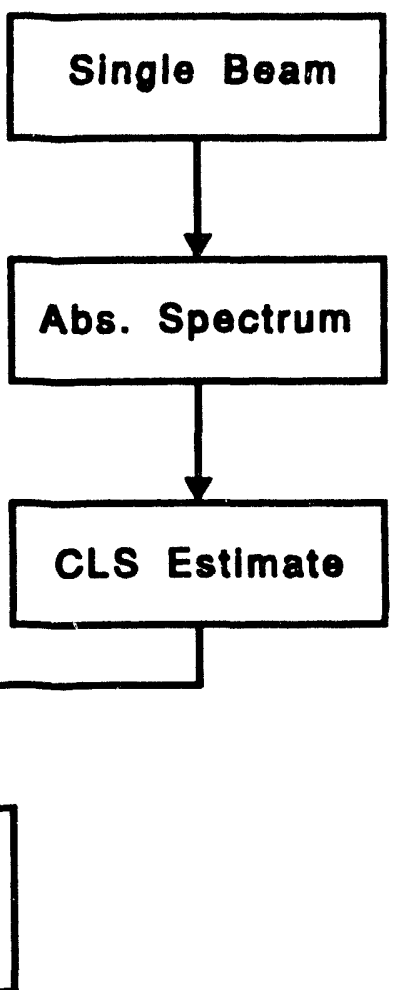

Fig. 7. CLS processing flowchart. 


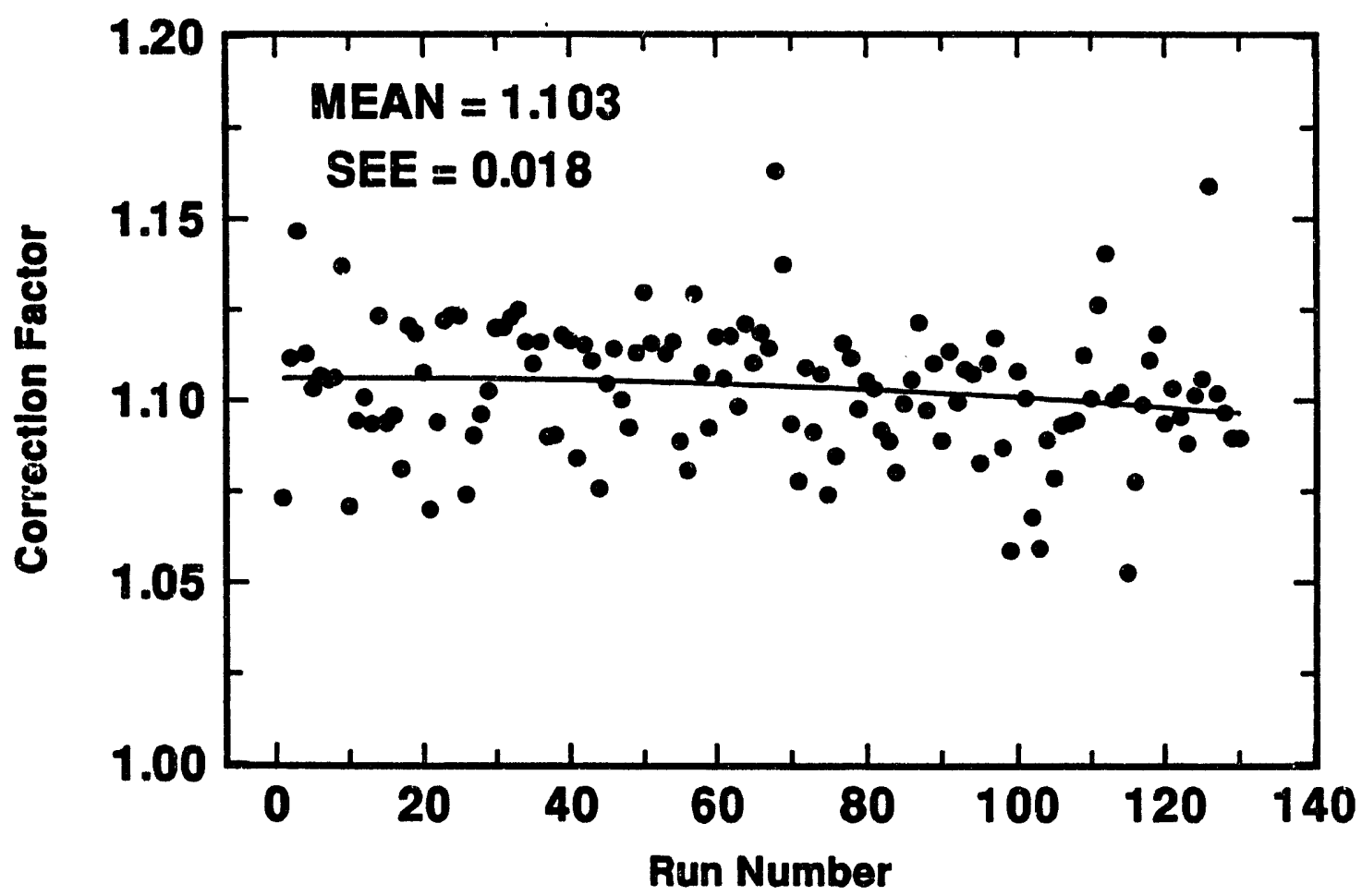

Fig. 8. Plot to determine the scale factor to apply to the CLS estimate of the background $\mathrm{H}_{2} \mathrm{O}$ concentration as derived from the TGS spectra. 


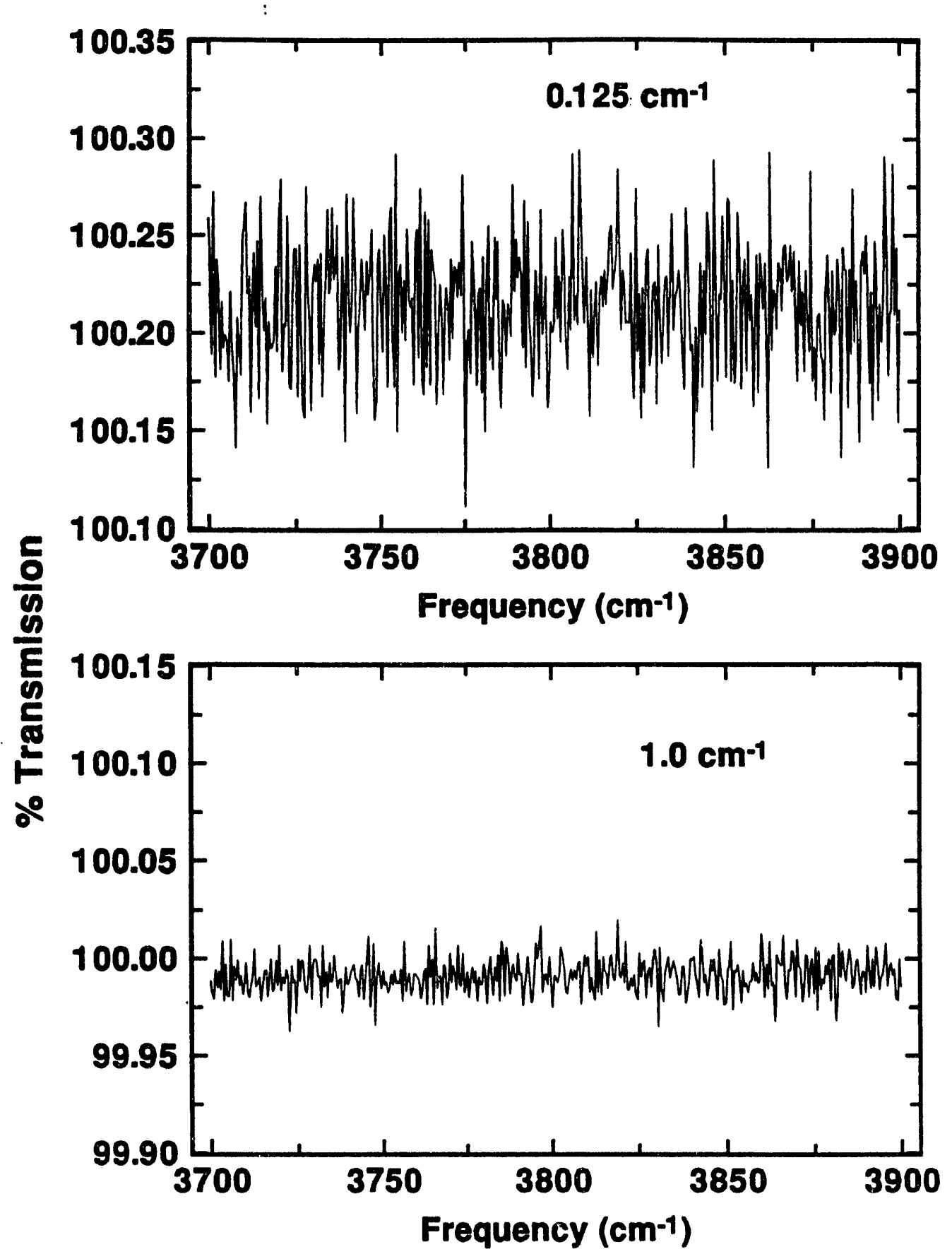

Fig. 9. Examples of $100 \%$ lines for $1 / 8 \mathrm{~cm}^{-1}$ and $1 \mathrm{~cm}^{-1}$ spectral resolutions. 


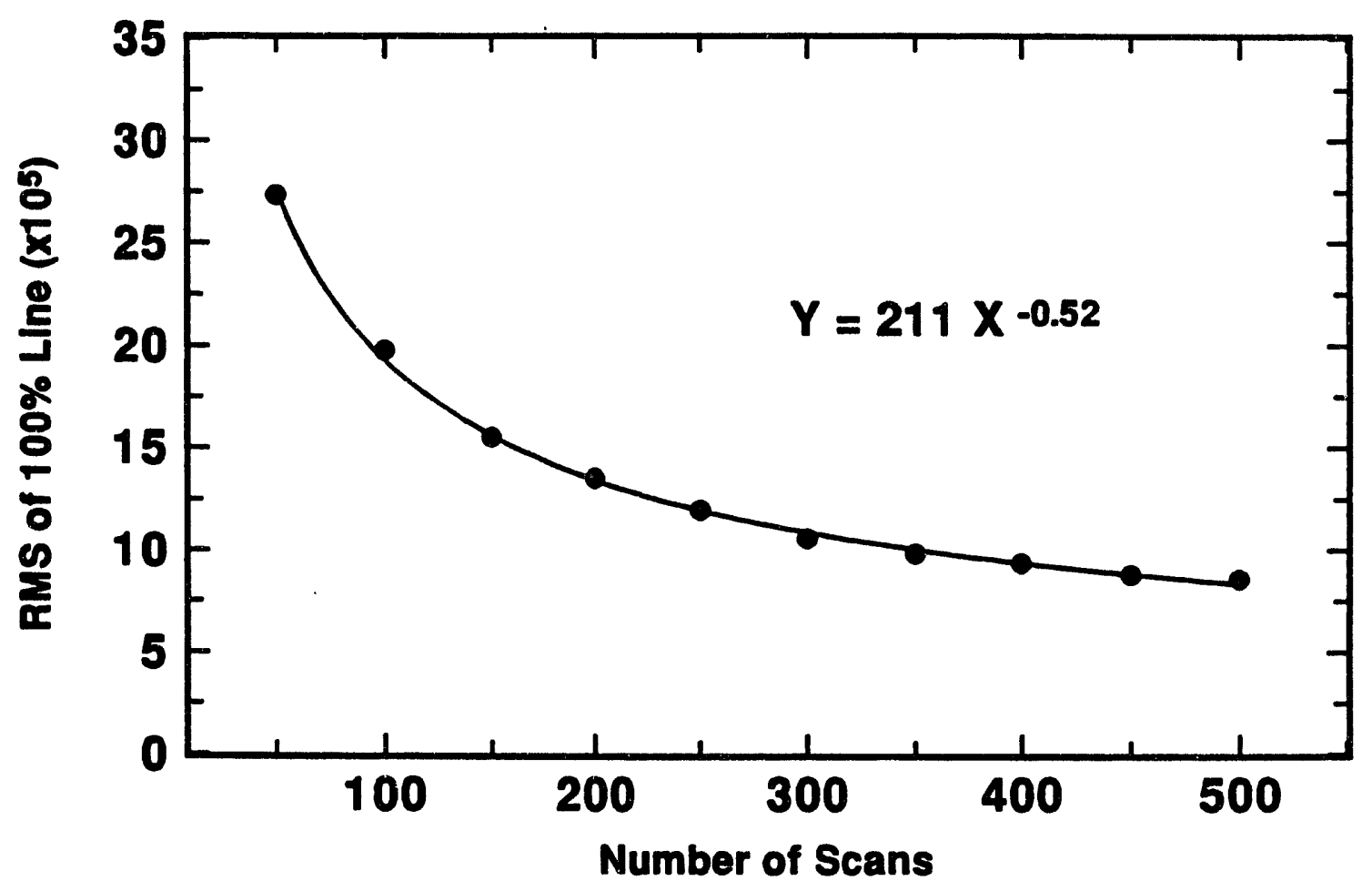

Fig. 10. Spectral noise vs. the number of accumulated scans for $1 \mathrm{~cm}^{-1}$ resolution. 


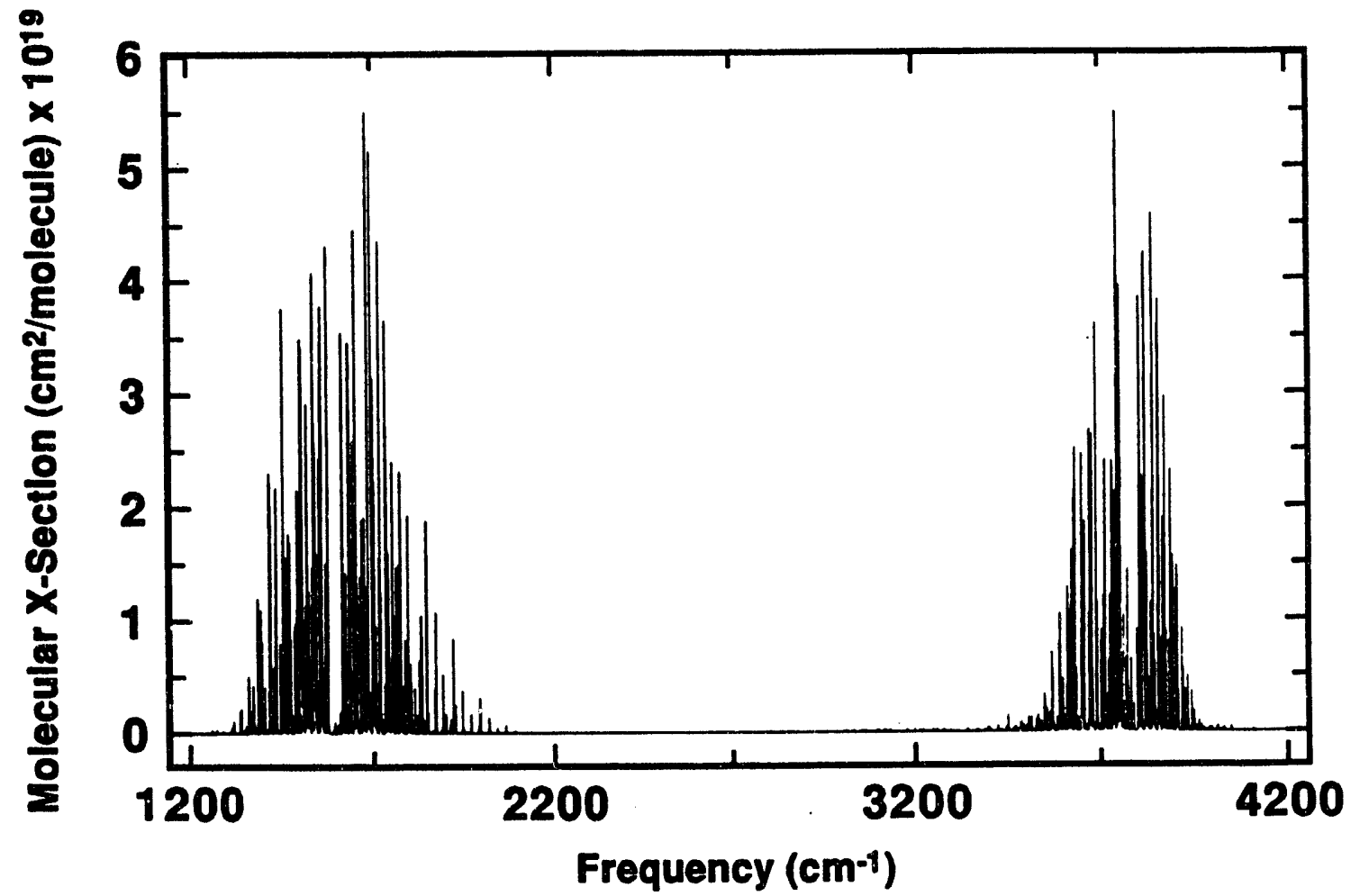

Fig. 11. Portion of the water vapor absorption spectrum showing two mid-ir absorption bands at $1600 \mathrm{~cm}^{-1}$ and $3800 \mathrm{~cm}^{-1}$. 


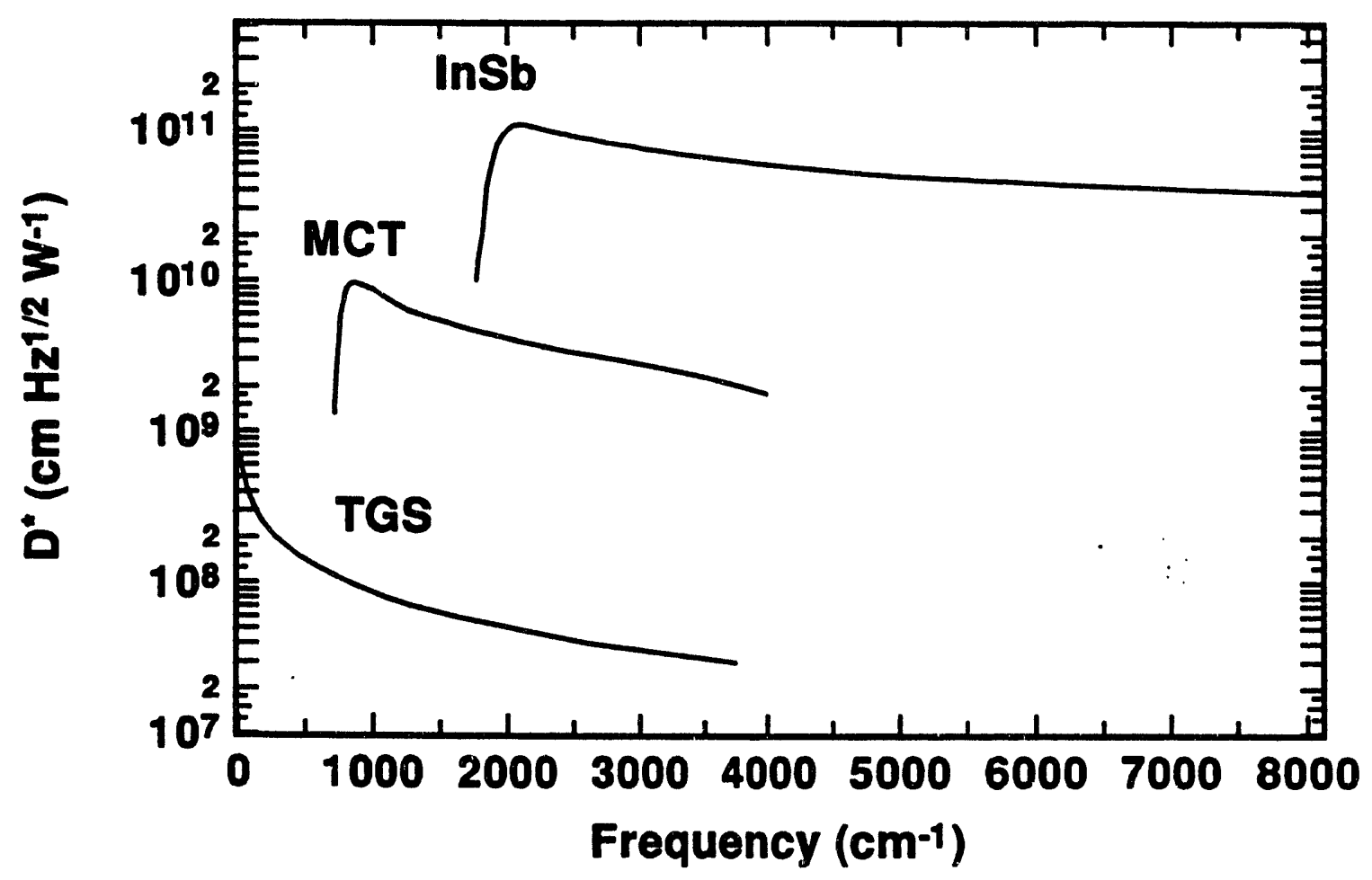

Fig. 12. Spectral specific detectivity $\left(D^{*}\right)$ for the detector materials InSb, MCT, and TGS. InSb is used in the photovoltaic mode, while MCT is used in the photoconductive mode. Both are cooled to $77 \mathrm{~K}$. TGS is at room temperature. 

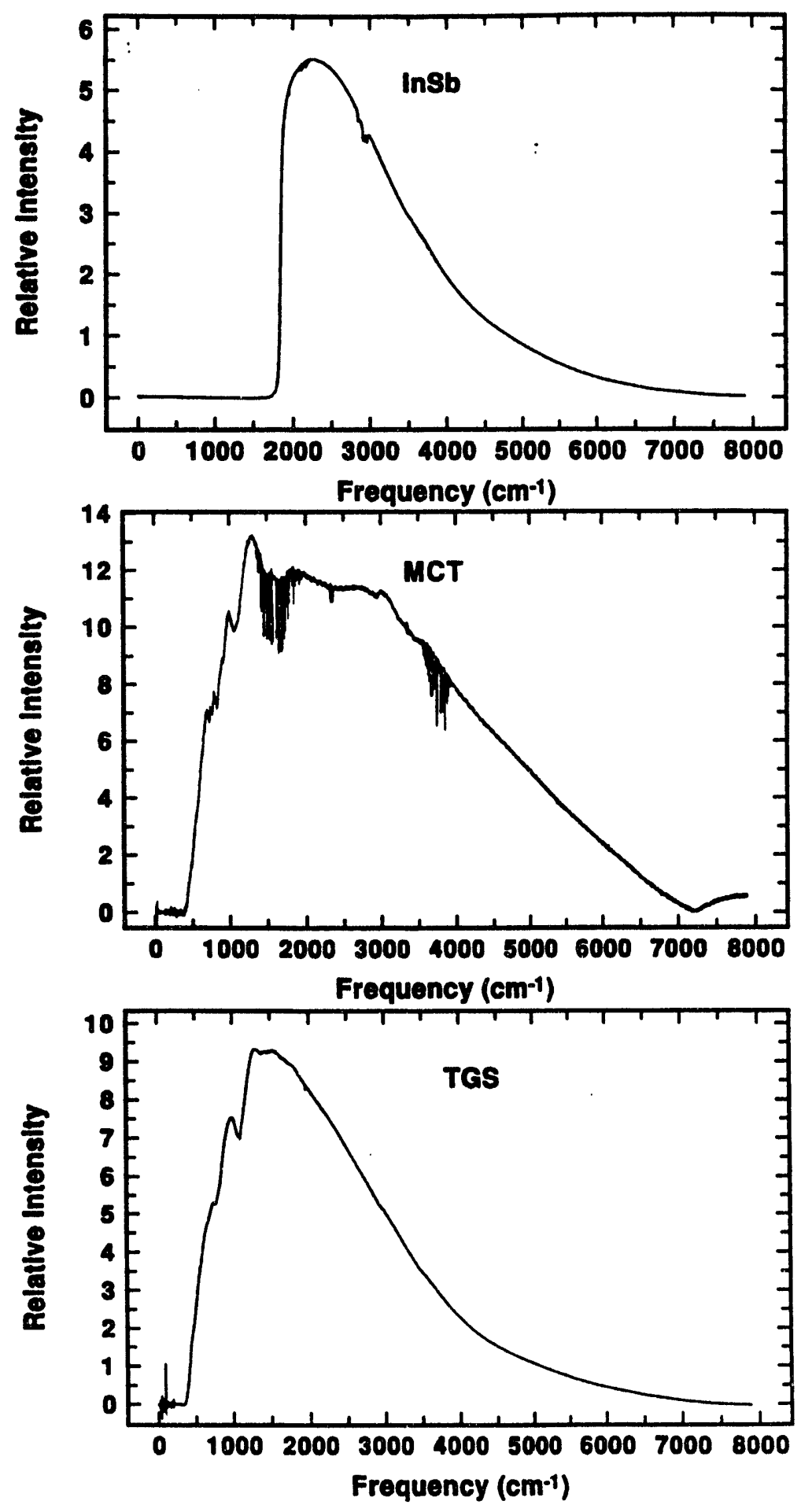

Fig. 13. Single beam specira for the detectors InSb, TGS, and MCT. The MCT spectrum was collected with a large amount of water vapor present to clearly show the two mid-ir absorption bands. 


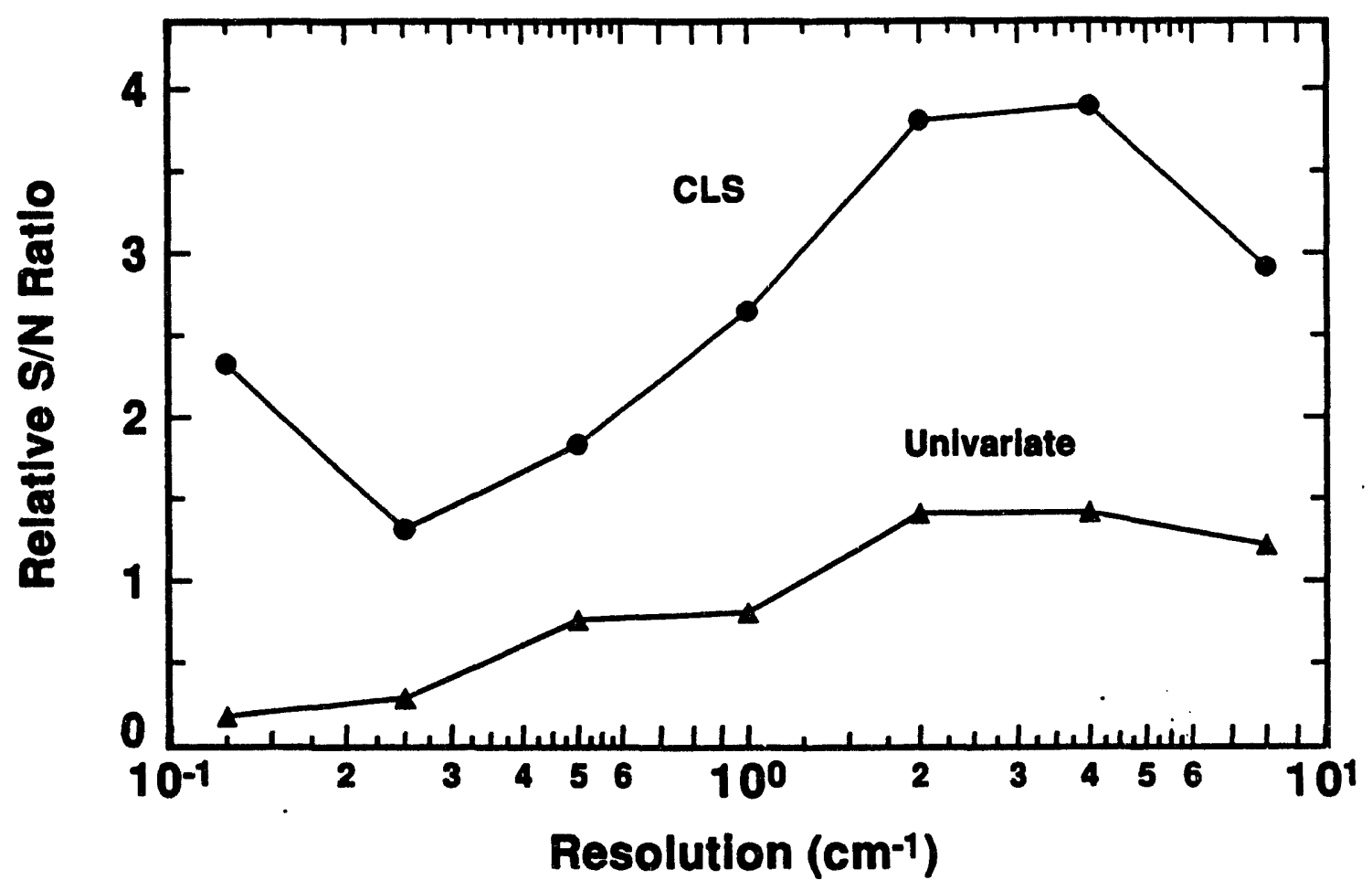

Fig. 14. Experimental results for a fixed scan time showing the relative SNR of the CLS and univariate calculations as a function of spectral resolution. The CLS calculation used the entire $\mathrm{H}_{2} \mathrm{O}$ spectrum from 3788 to $3910 \mathrm{~cm}^{-1}$, while univariate calculation used the peak height of the $3837.9 \mathrm{~cm}^{-1}$ band. 


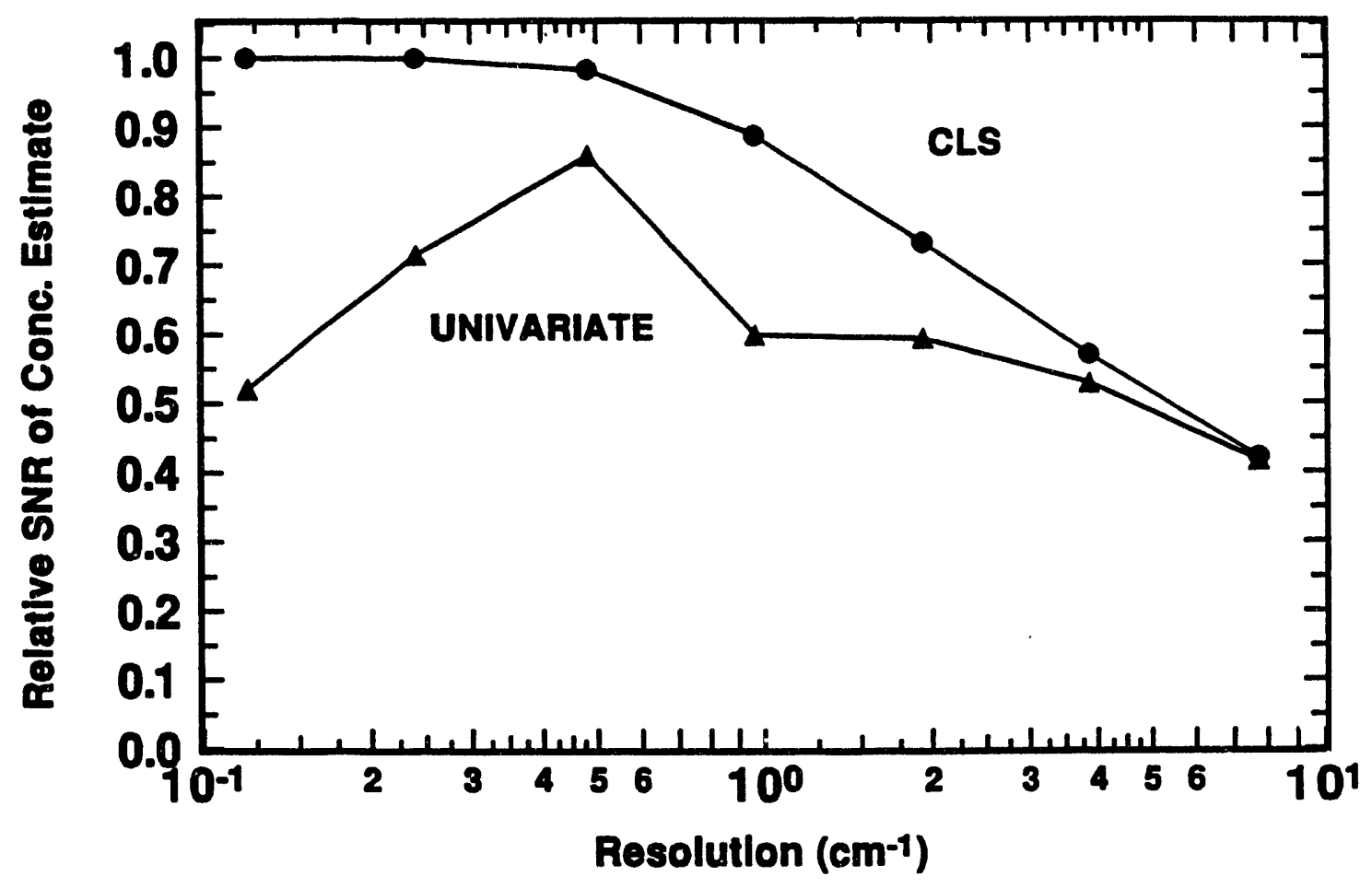

Fig. 15. Theoretical results showing the relative SNR of the CLS and univariate estimators as a function of the spectral resolution. The numerical model assumes a spectrum consisting of a single, isolated absorption line (FWHM $\approx 0.2 \mathrm{~cm}-1$ ) and an ic'eal FTIR spectrometer set to accumulate a fixed number of scans at each spectral resolution. 


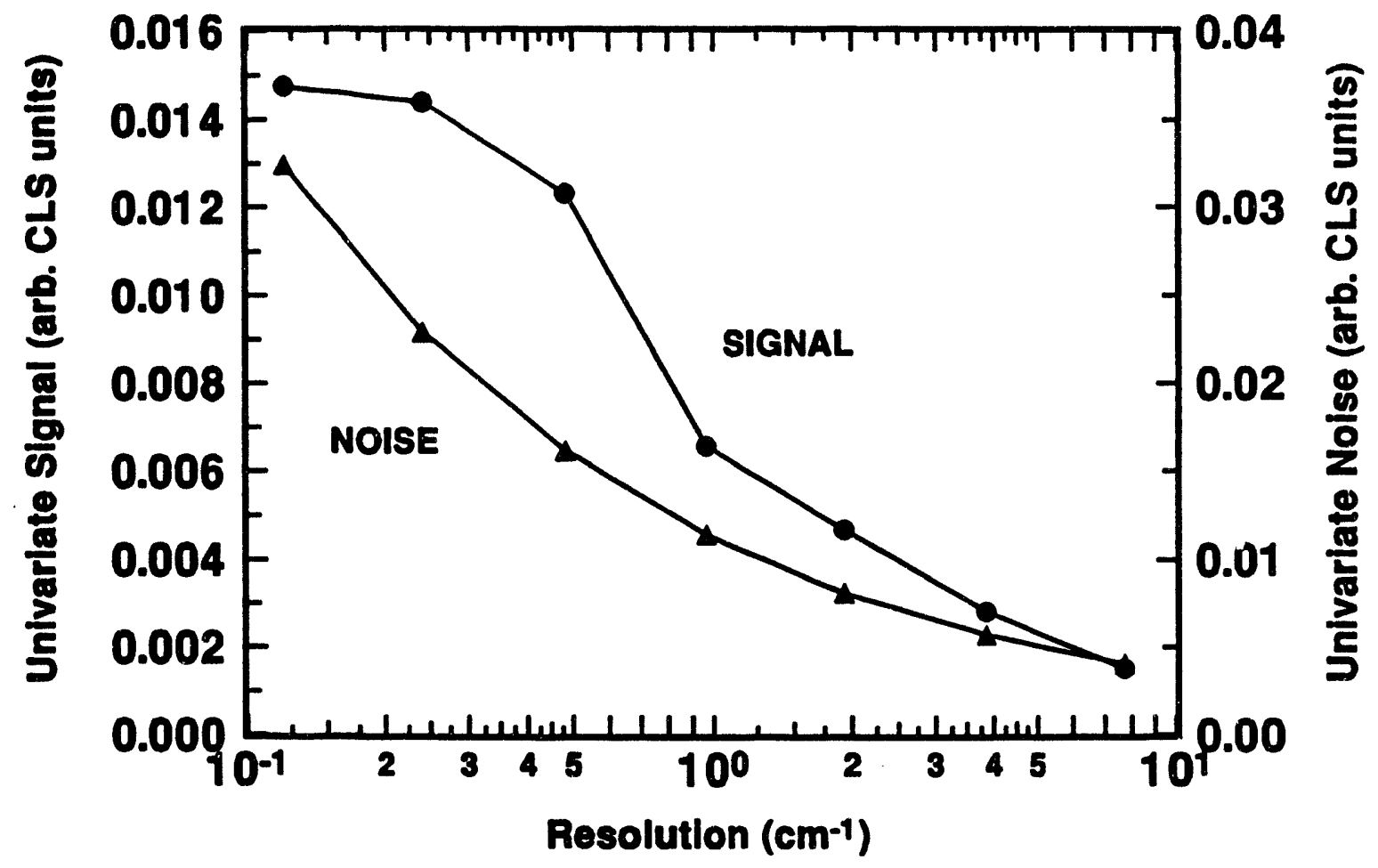

Fig. 16. Theoretical results showing the univariate signal (i.e., peak height) and the spectral noise as a function of the spectral resolution. The numerical model is identical to the one used to produce the data shown in Fig. 15. 


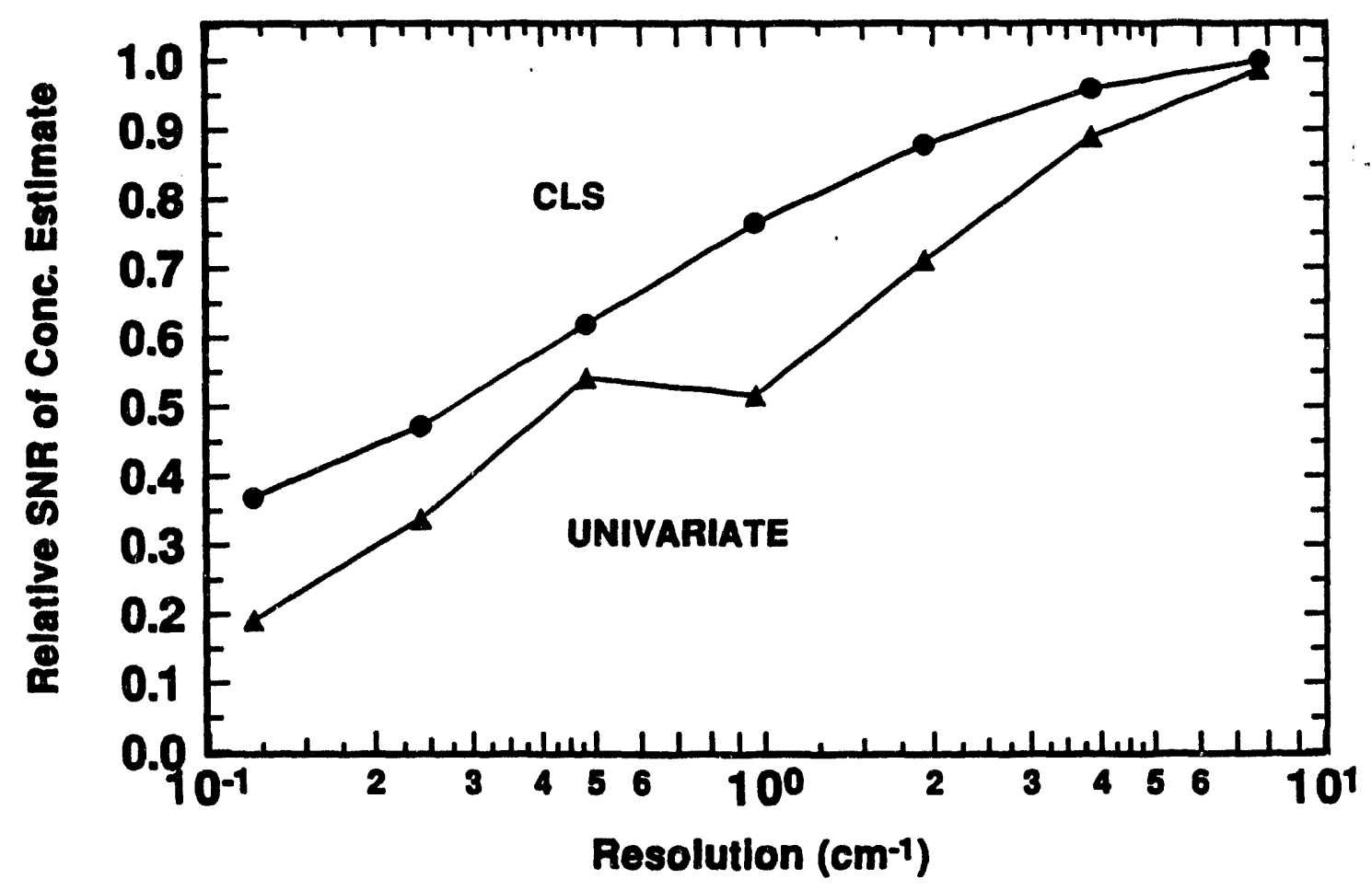

Fig. 17. Theoretical results showing the relative SNR of CLS and univariate estimators as a function of the spectral resolution. The numerical model assumes a spectrum consisting of a single, isolated absorption line (FWHM $\approx 0.2 \mathrm{~cm}-1$ ) and an ideal FTIR spectrometer set to accumulate scans for a fixed time at each spectral resolution. 


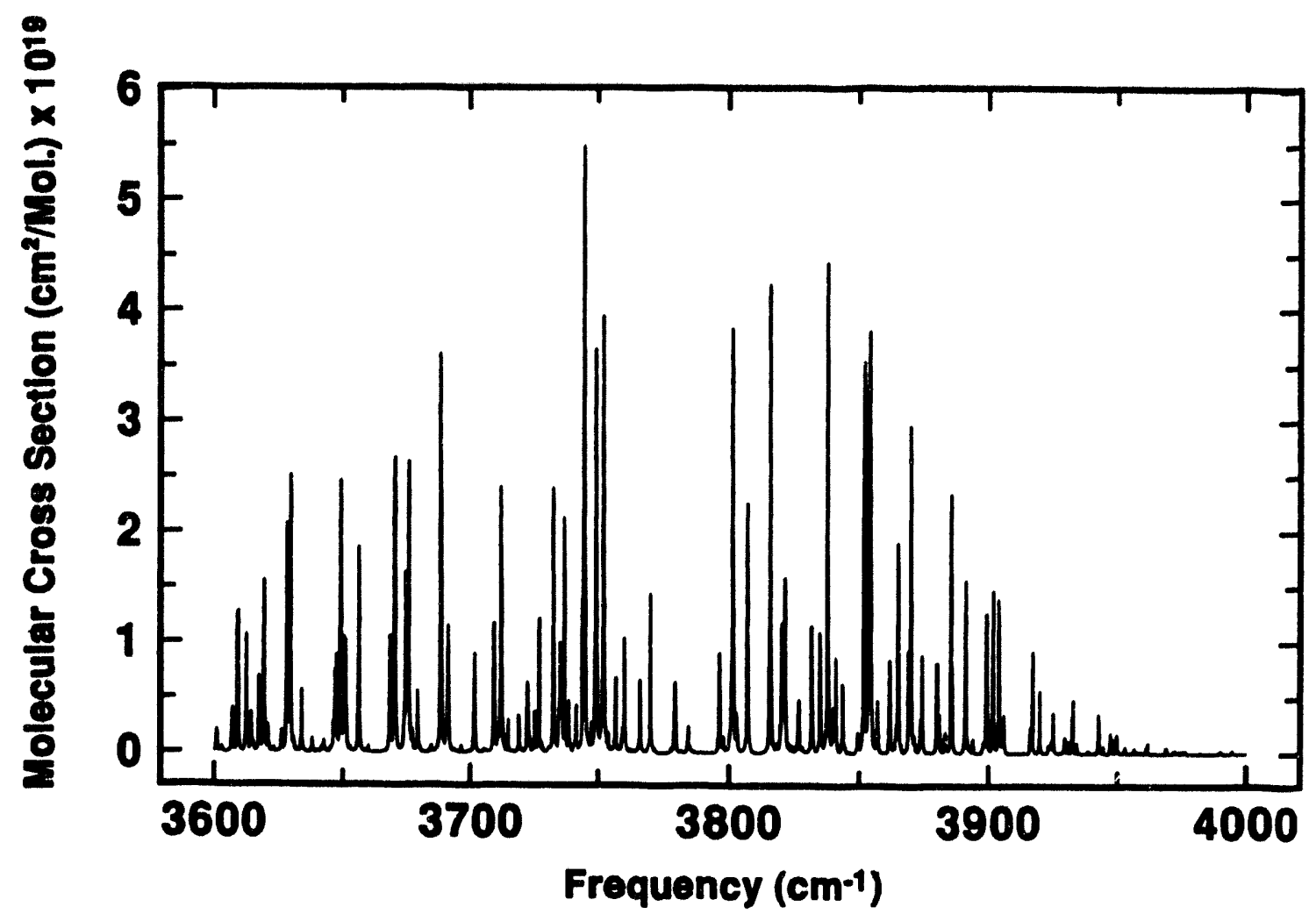

Fig. 18. The relevant portion of the HITRAN water vapor absorption spectrum shown in units of the molecular cross-section. 


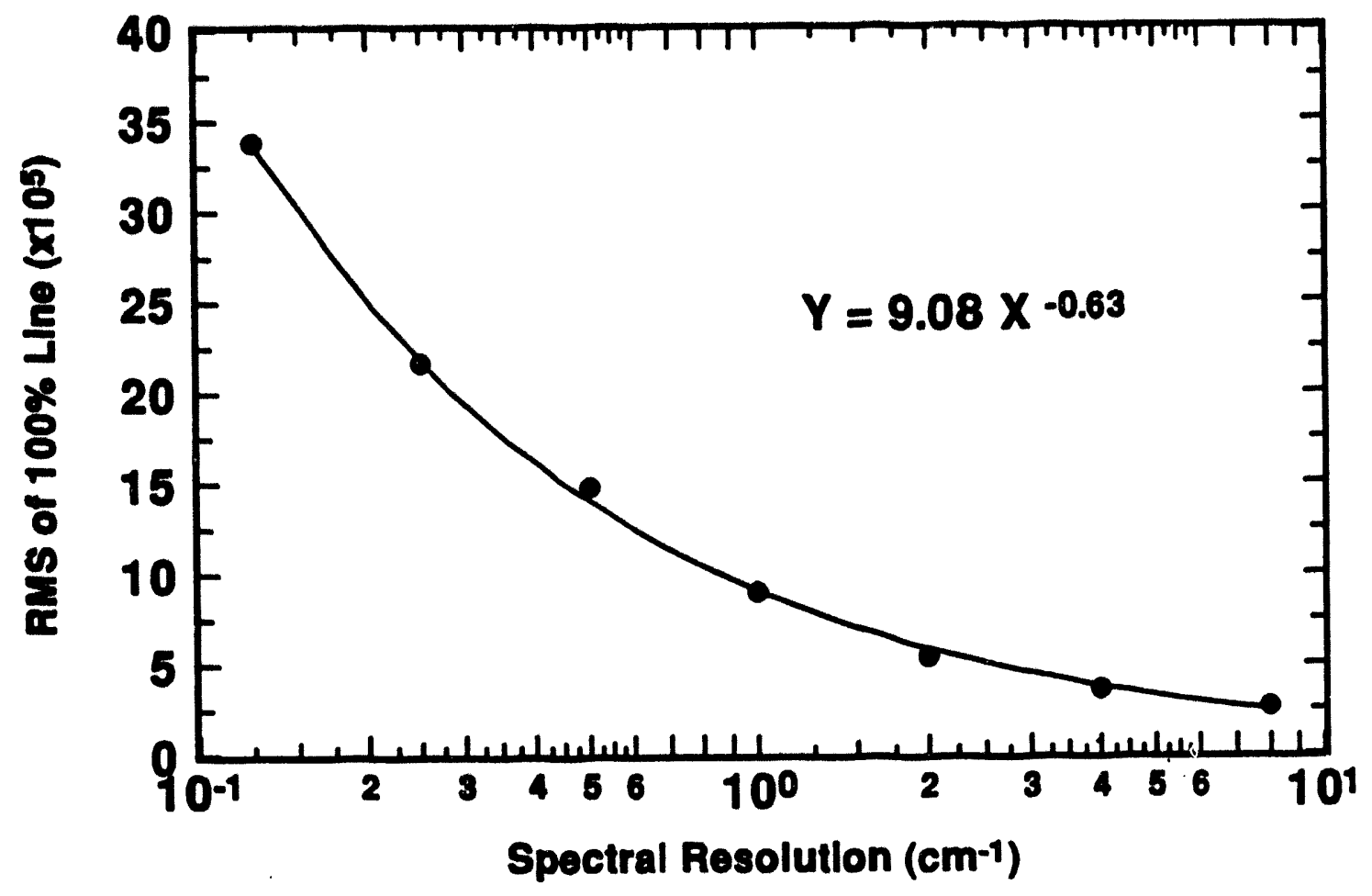

Fig. 19. Experimental data showing the spectral noise as a function of resolution for a fixed number of scans. 


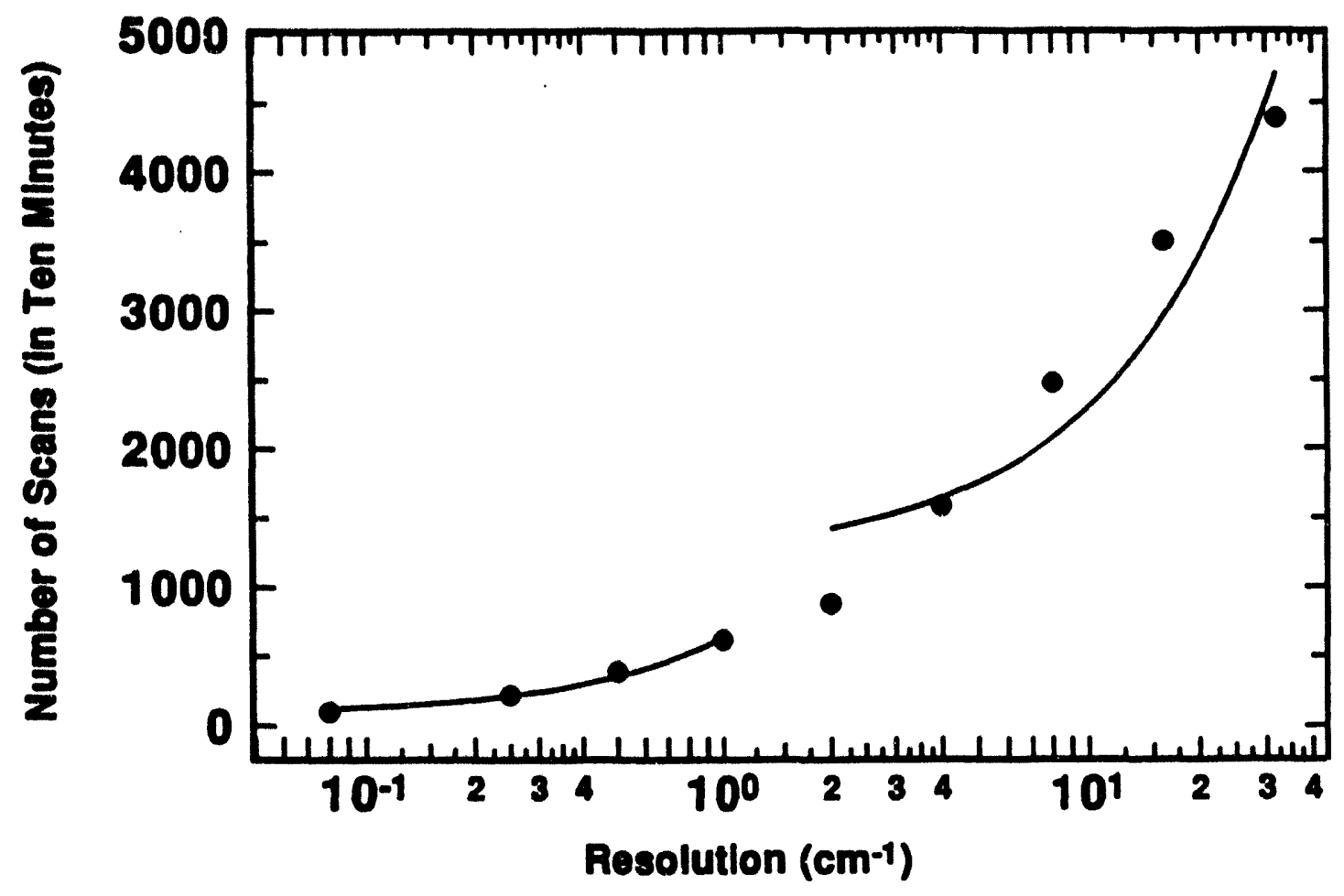

Fig. 20. Experimental data showing the number of scans in a 10 minute accumulation time as a function of the spectral resolution. Also shown are best-fit lines in the two collection regions: single-sided interferograms at resolutions $\leq 1.0 \mathrm{~cm}^{-1}$, and double-sided interferograms at resolutions $\geq 2.0 \mathrm{~cm}^{-1}$. Note that the appearance of linearity is lost because of the log scale. 


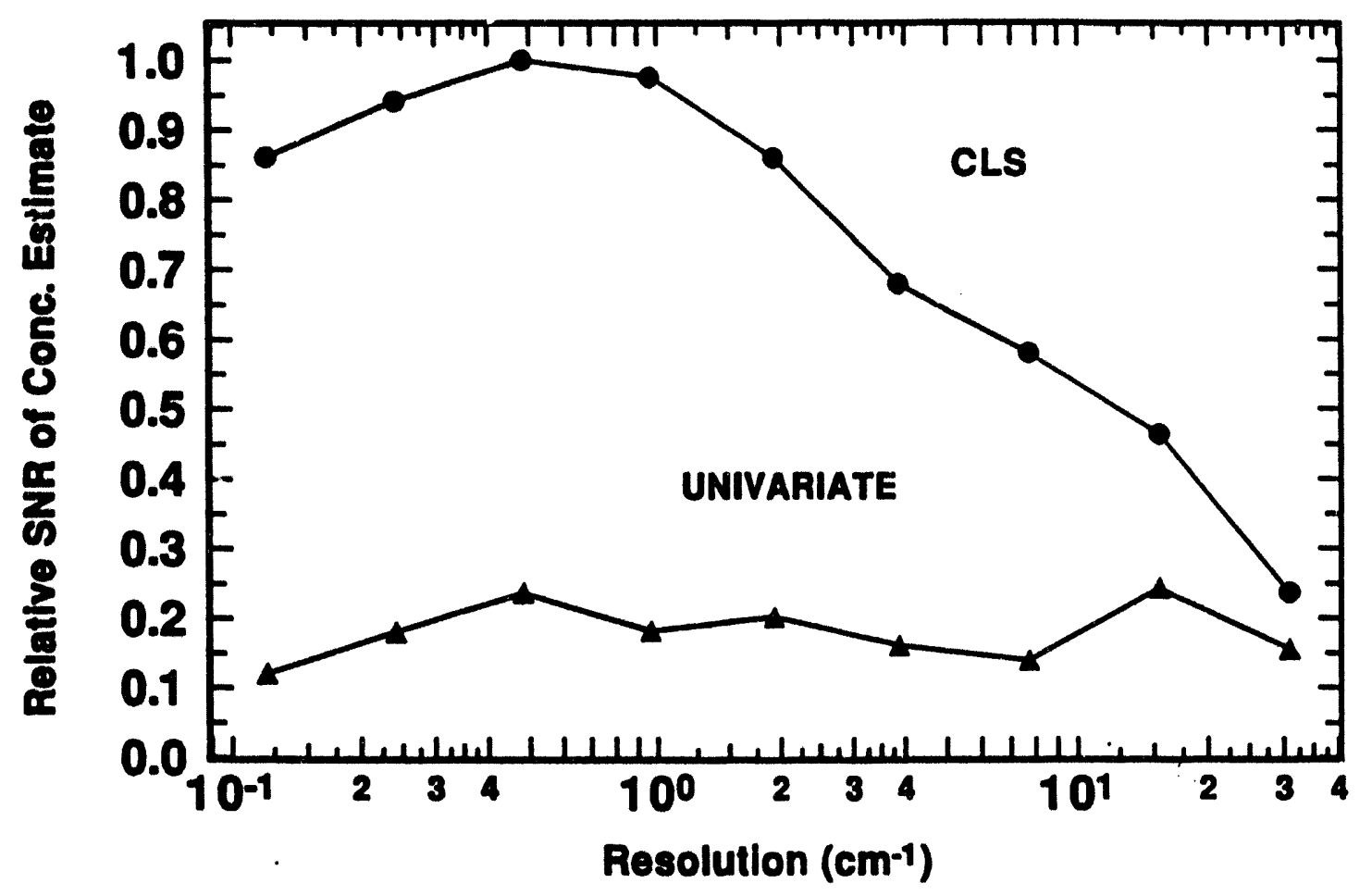

Fig. 21. Theoretical results showing the relative SNR of CLS and univariate estimators as a function of the spectral resolution for a fixed number of scans. The numerical model assumes a realistic water vapor spectrum (as shown in Fig. 18) in the region from 3788 to $3910 \mathrm{~cm}^{-1}$, and the spectrometer noise and scan overhead characteristics shown in Figs. 19 and 20. 


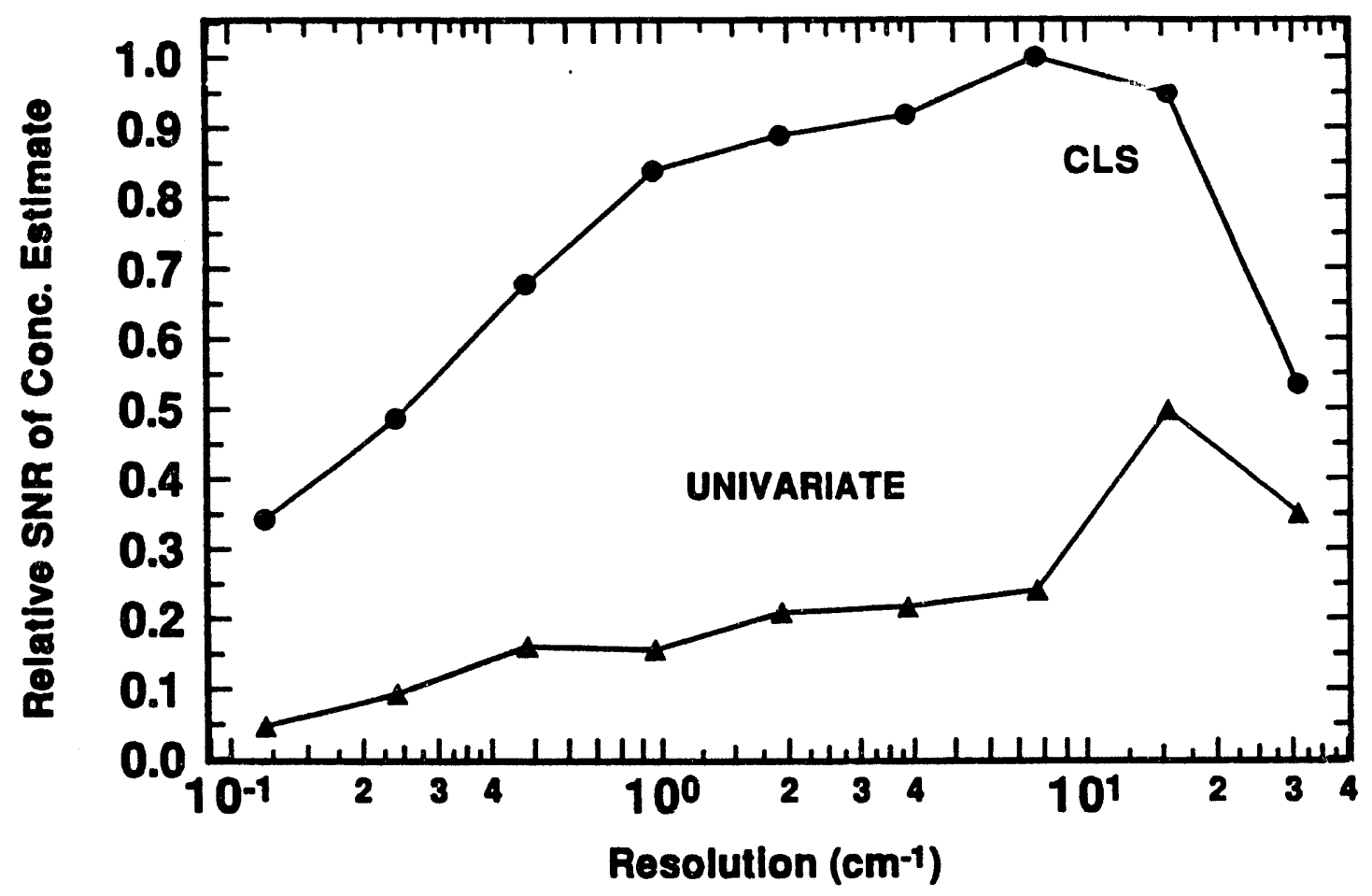

Fig. 22. Theoretical results showing the relative SNR of CLS and univariate estimators as a function of the spectral resolution for a fixed collection time interval. The numerical model assumes a realistic water vapor spectrum (as shown in Fig. 18) in the region from 3788 to $3910 \mathrm{~cm}^{-1}$, and the spectrometer noise and scan overhead characteristics shown in Figs. 19 and 20. This figure depicts the results from the numerical model that is most similar to the actual experimental conditions that produced the results shown in Fig. 14. 


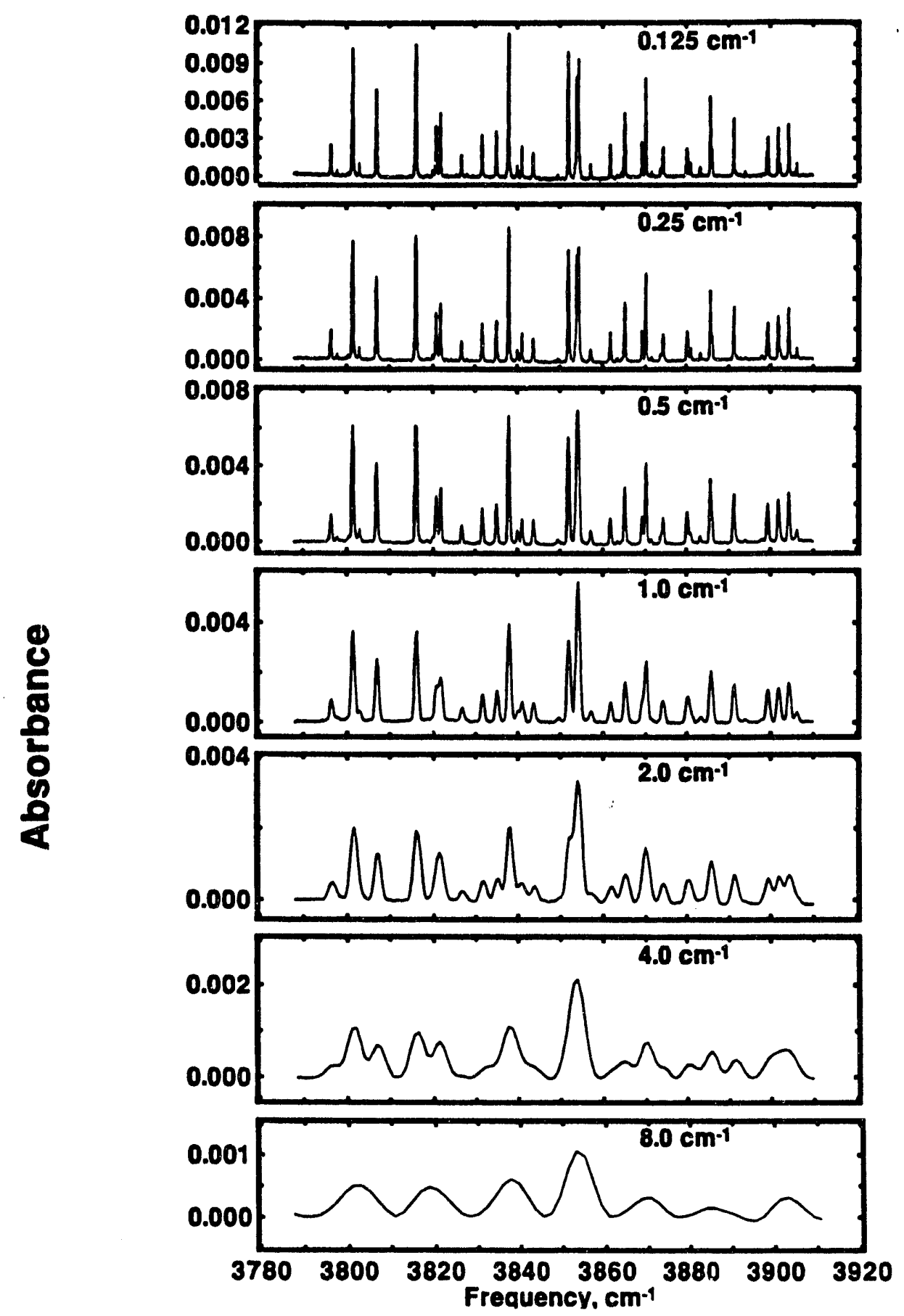

Fig. 23. A series of $\mathrm{H}_{2} \mathrm{O}$ absorption spectra at different spectral resolutions. 

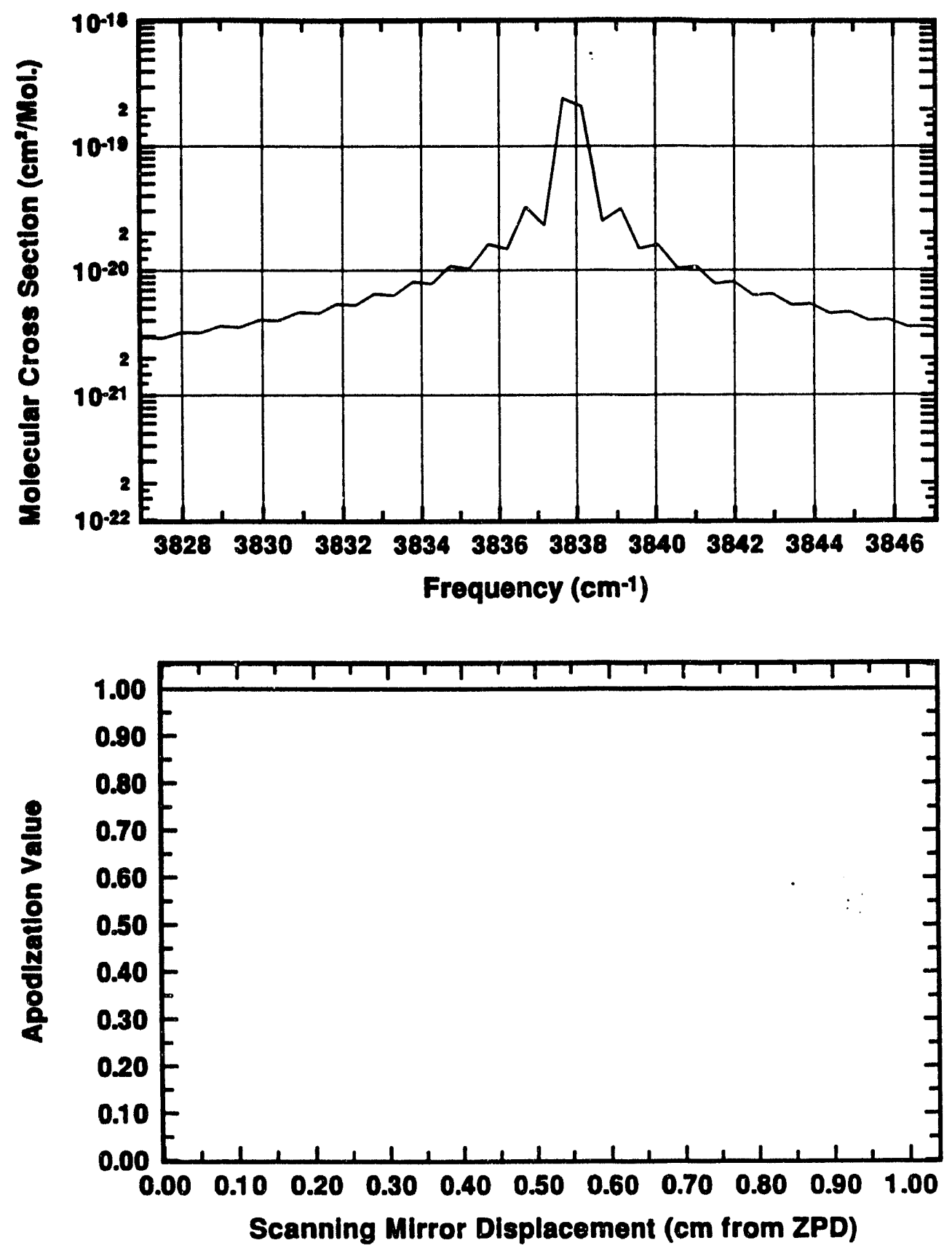

Fig. 24. Top - Simulated FTIR absorption spectrum of an isolated absorption line, $1 \mathrm{~cm}^{-1}$ resolution, and boxcar apodization. Bottom - Plot of the boxcar apodization function. 

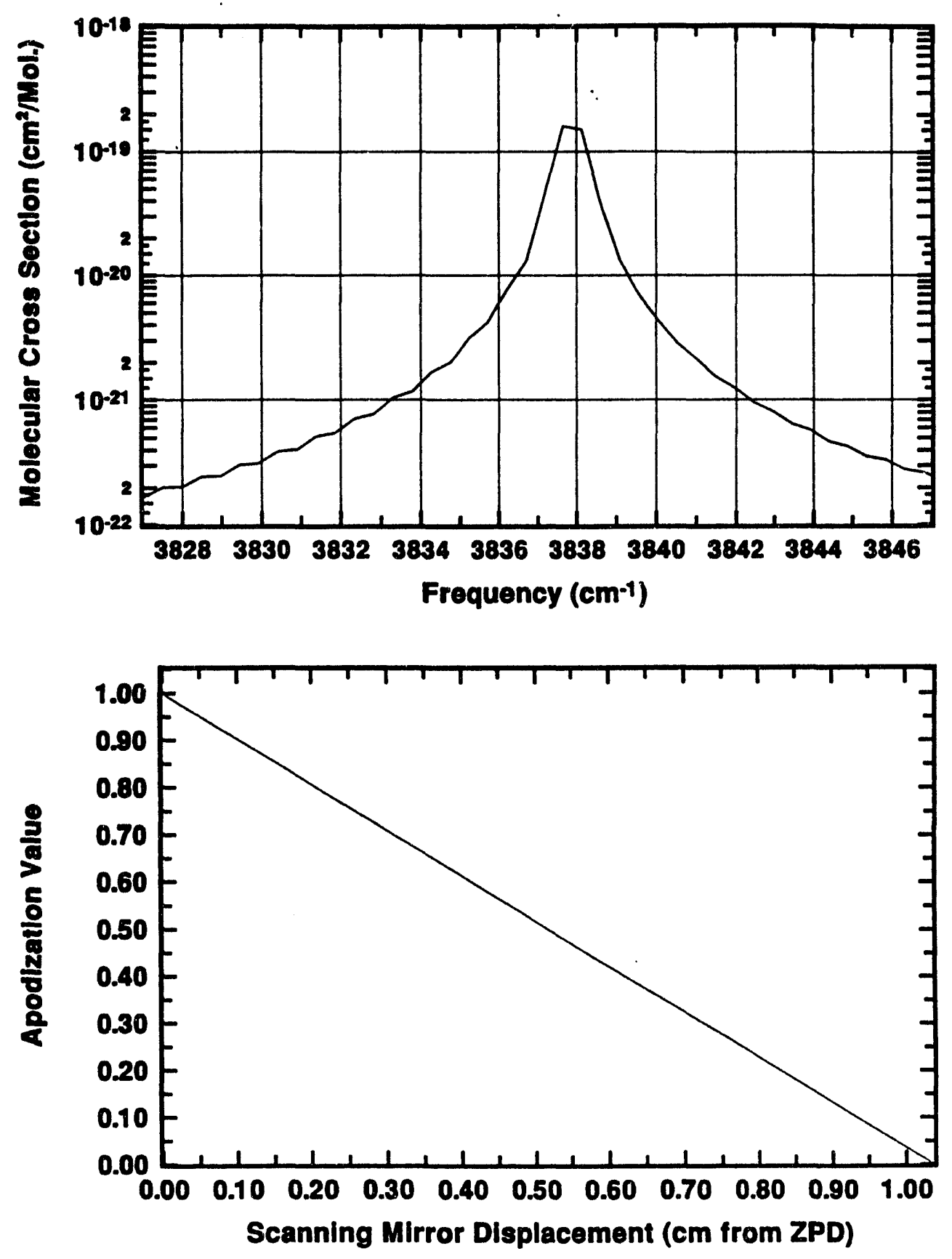

Fig. 25. Top - Simulated FTIR absorption spectrum of an isolated absorption line, $1 \mathrm{~cm}^{-1}$ resolution, and triangular apodization. Bottom - Plot of the triangular apodization function. 

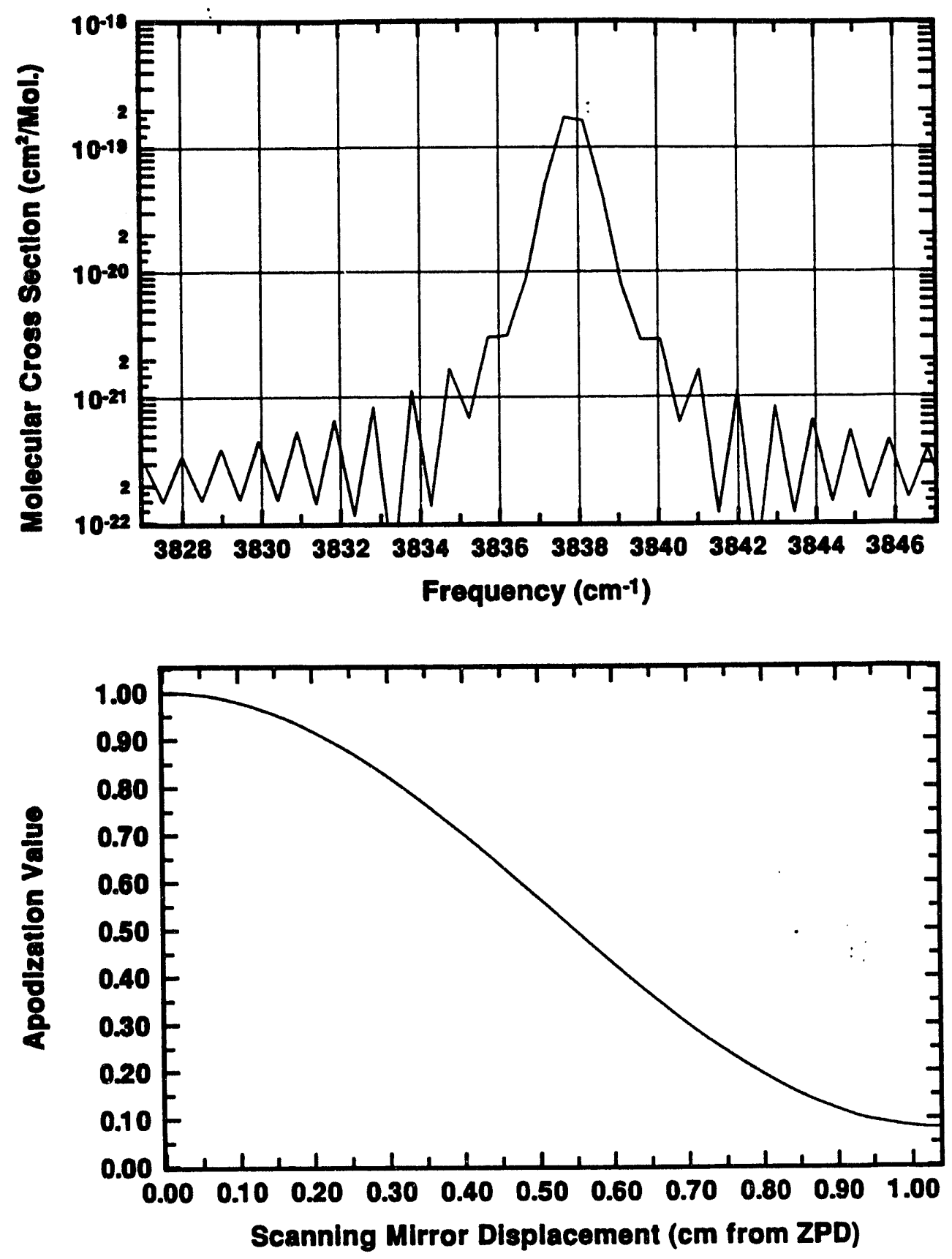

Fig. 26. Top - Simulated FTIR absorption spectrum of an isolated absorption line, $1 \mathrm{~cm}^{-1}$ resolution, and Happ-Genzel apodization. Bottom - Plot of the Happ-Genzel apodization function. 


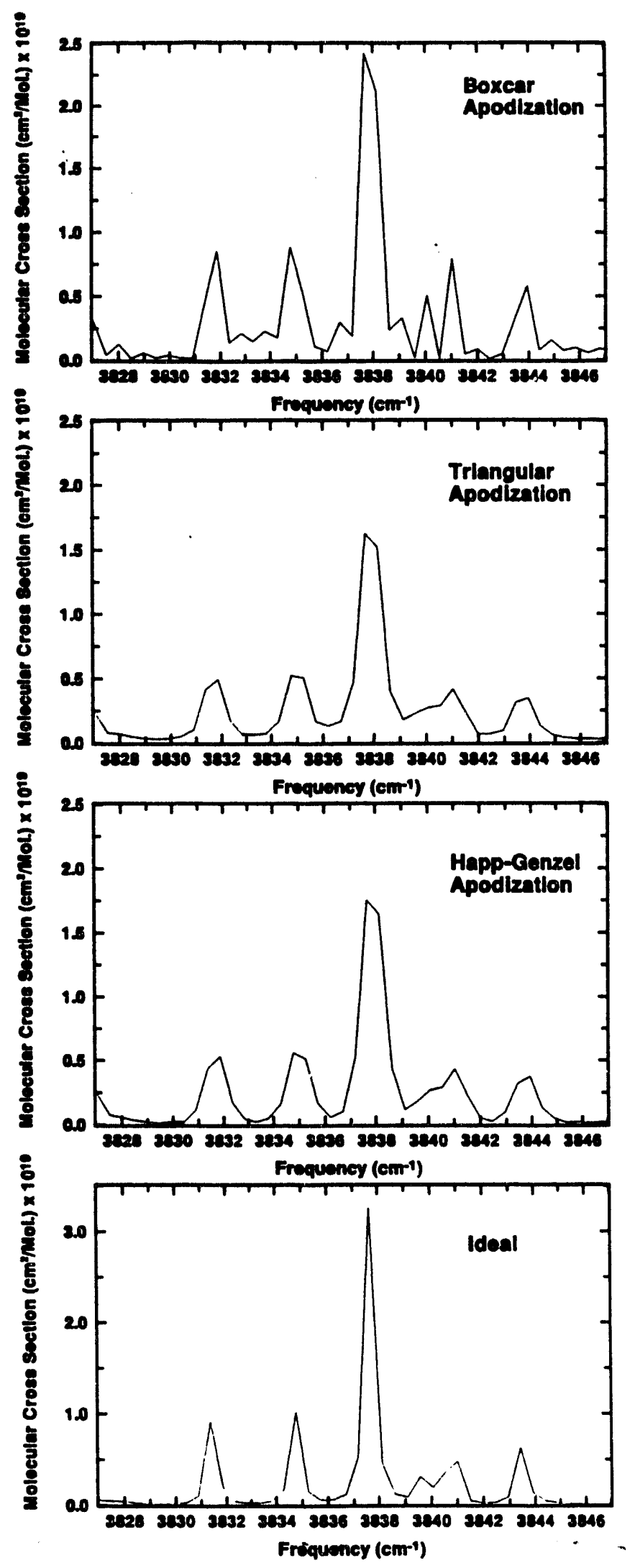

Fig 27. Portion of a simulated water vapor absorption spectra with $1 \mathrm{~cm}^{-1}$ resolution showing boxcar, triangular, and Happ-Genzel apodization. The ideal spectrum at the bottom has infinite resolution and is binned at intervals of $0.48 \mathrm{~cm}^{-1}$ to be comparable to previous spectra. 


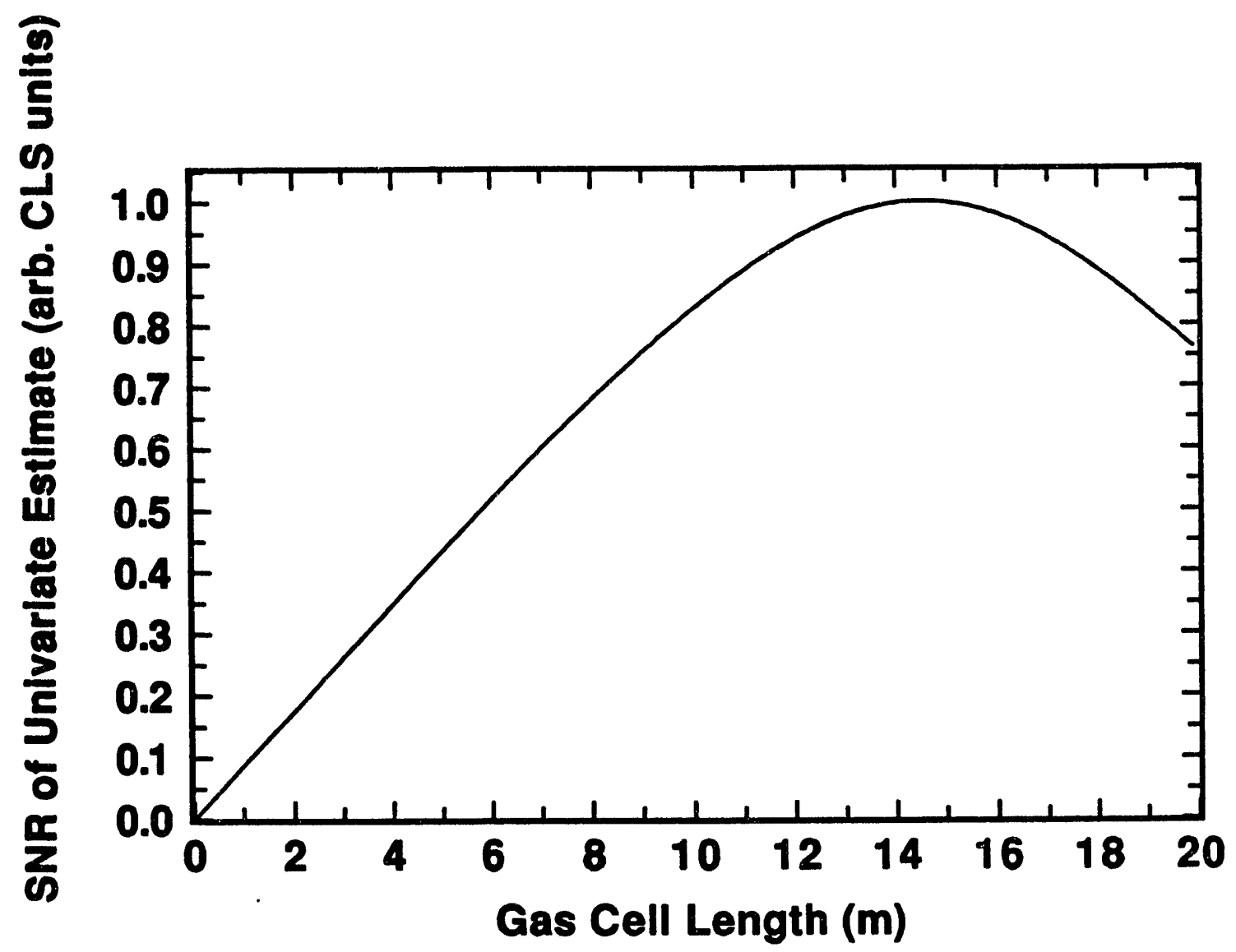

Fig. 28. Theoretical plot of SNR vs. Axiom cell path length, according to Eq. 24, for the conditions described in the text. 


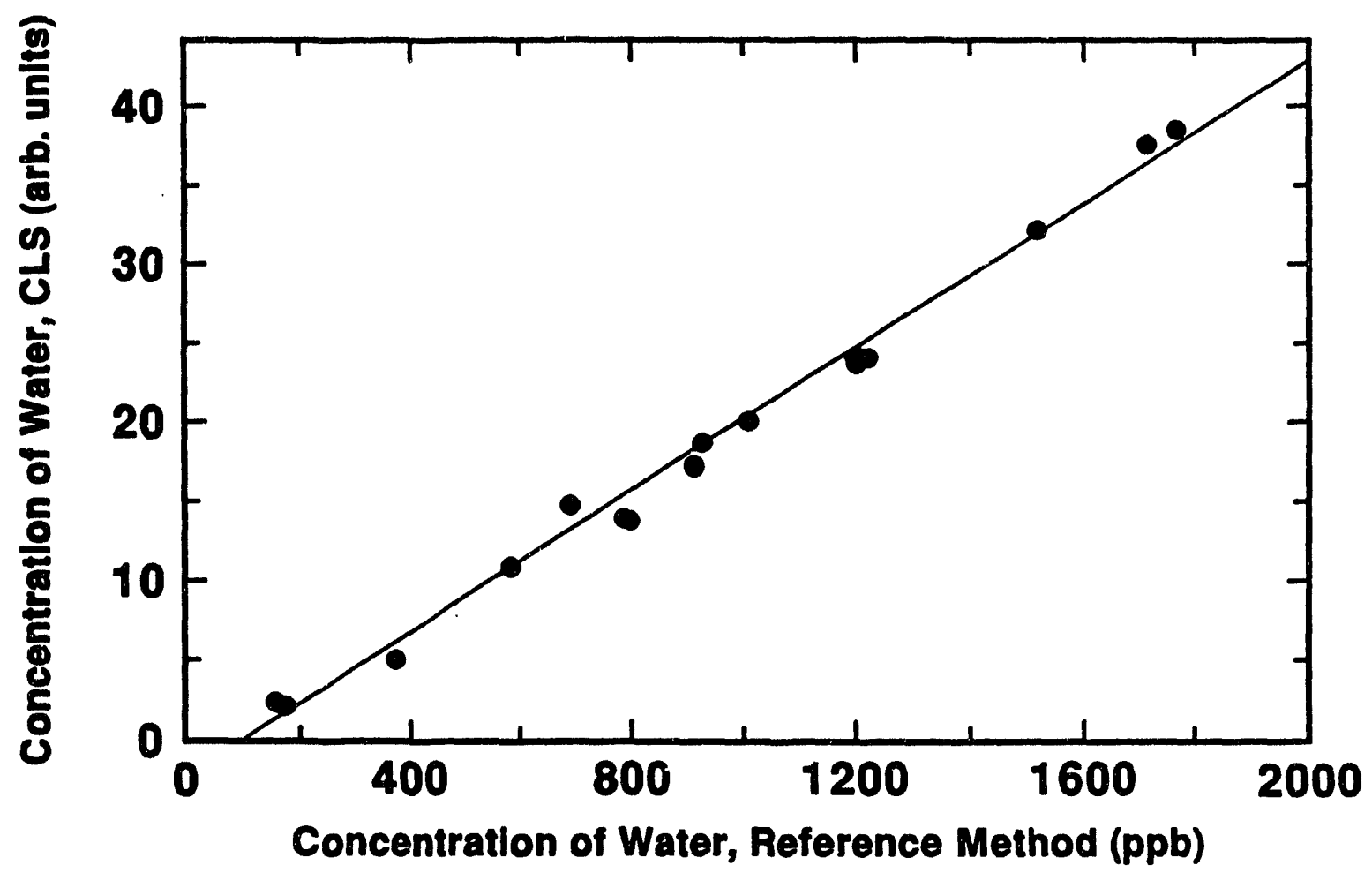

Fig. 29. Measured $\mathrm{H}_{2} \mathrm{O}$ concentration in $\mathrm{N}_{2}$ vs. the reference values from the Aquamatic Plus. Data were collected with the White cell. The solid line is a linear regression., 


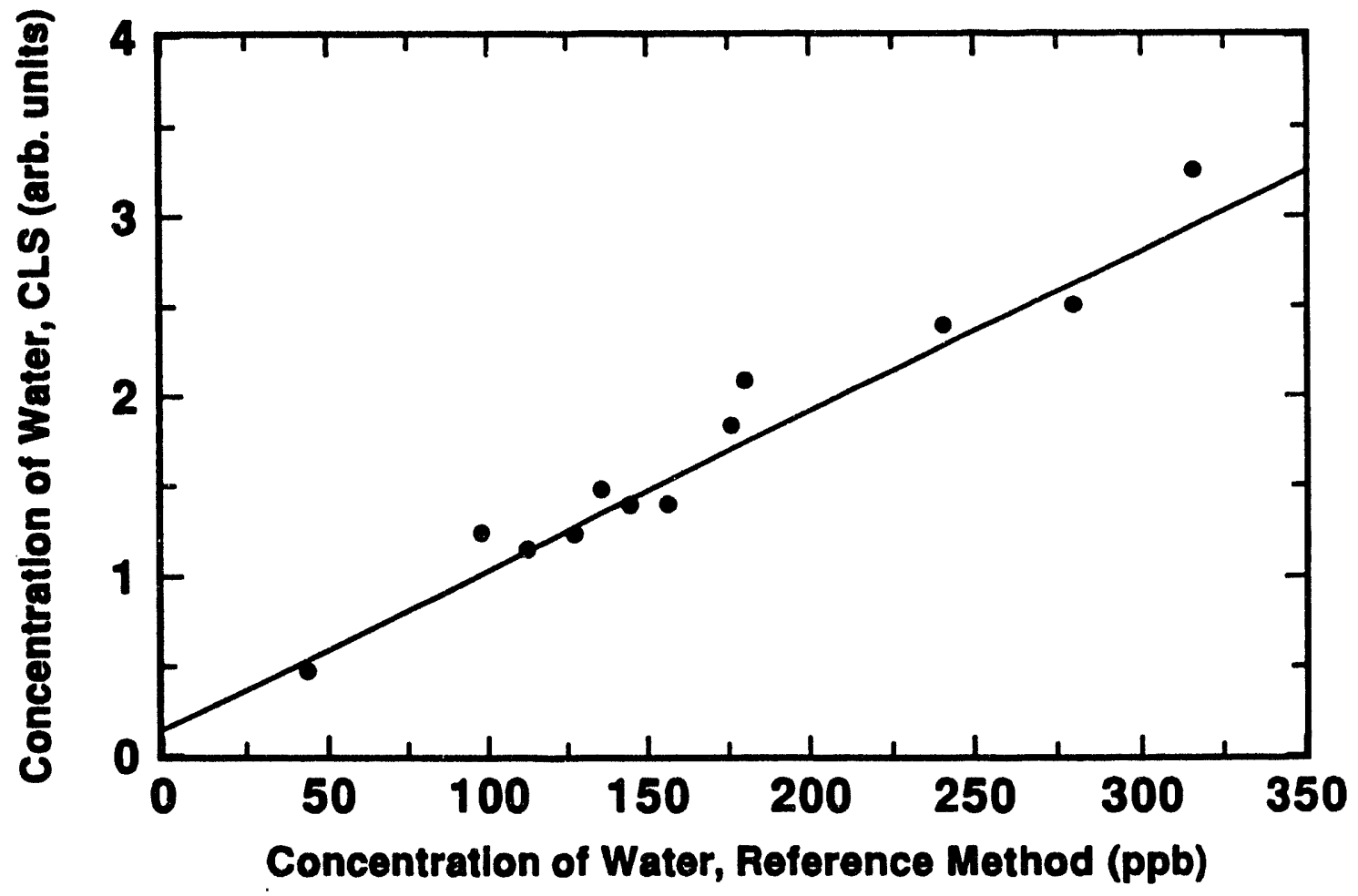

Fig. 30. Measured $\mathrm{H}_{2} \mathrm{O}$ concentration in $\mathrm{N}_{2}$ vs. the reference values from the Aquamatic Plus. Data were collected with the Axiom cell. The solid line is a linear regression. 


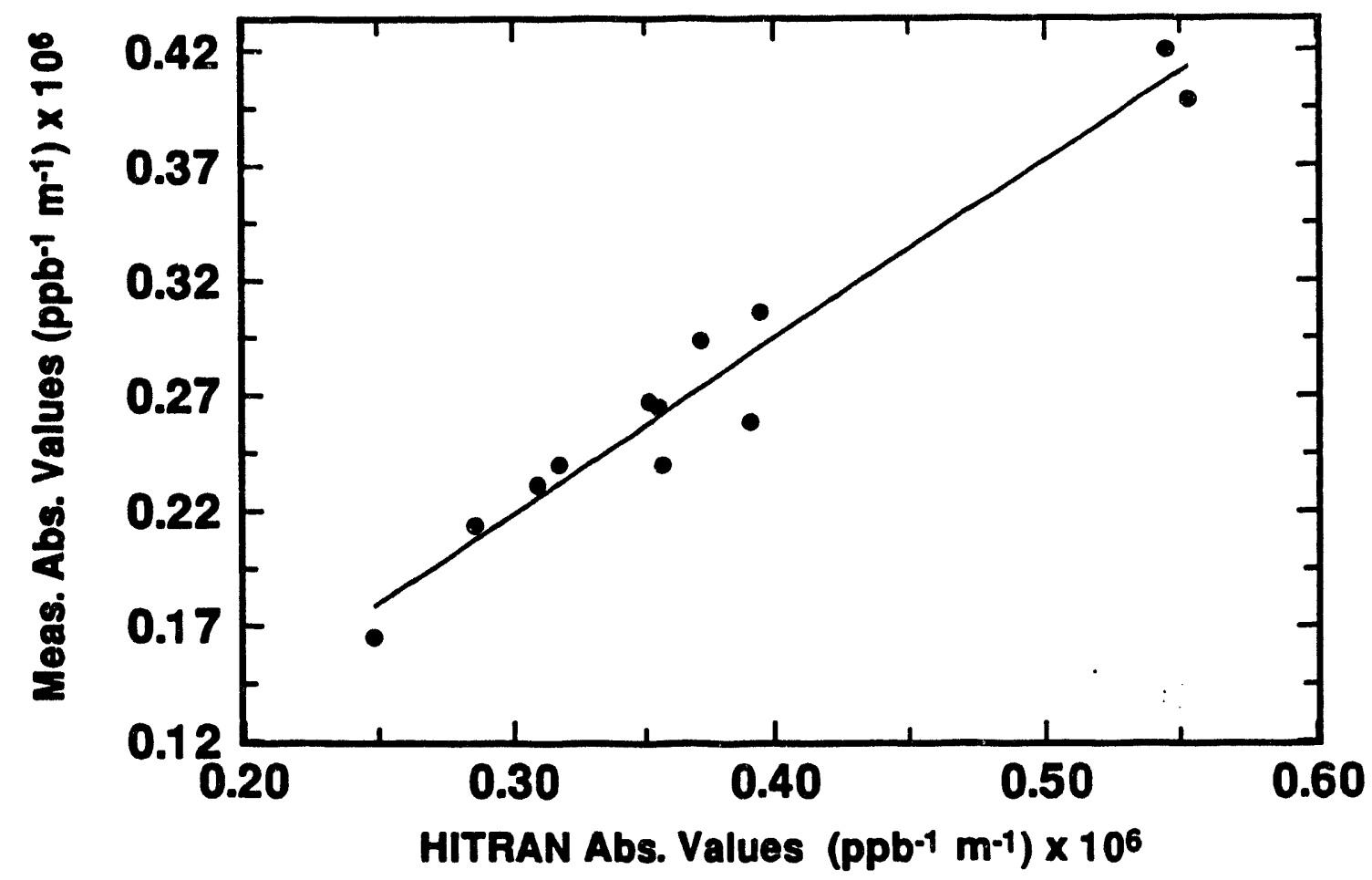

Fig. 31. A plot of experimental absorptivities for twelve prominent $\mathrm{H}_{2} \mathrm{O}$ bands in the 3800 $\mathrm{cm}^{-1}$ region vs. absorptivities derived from HITRAN for the same conditions. The solid line is a linear regression going essentially through the origin. 


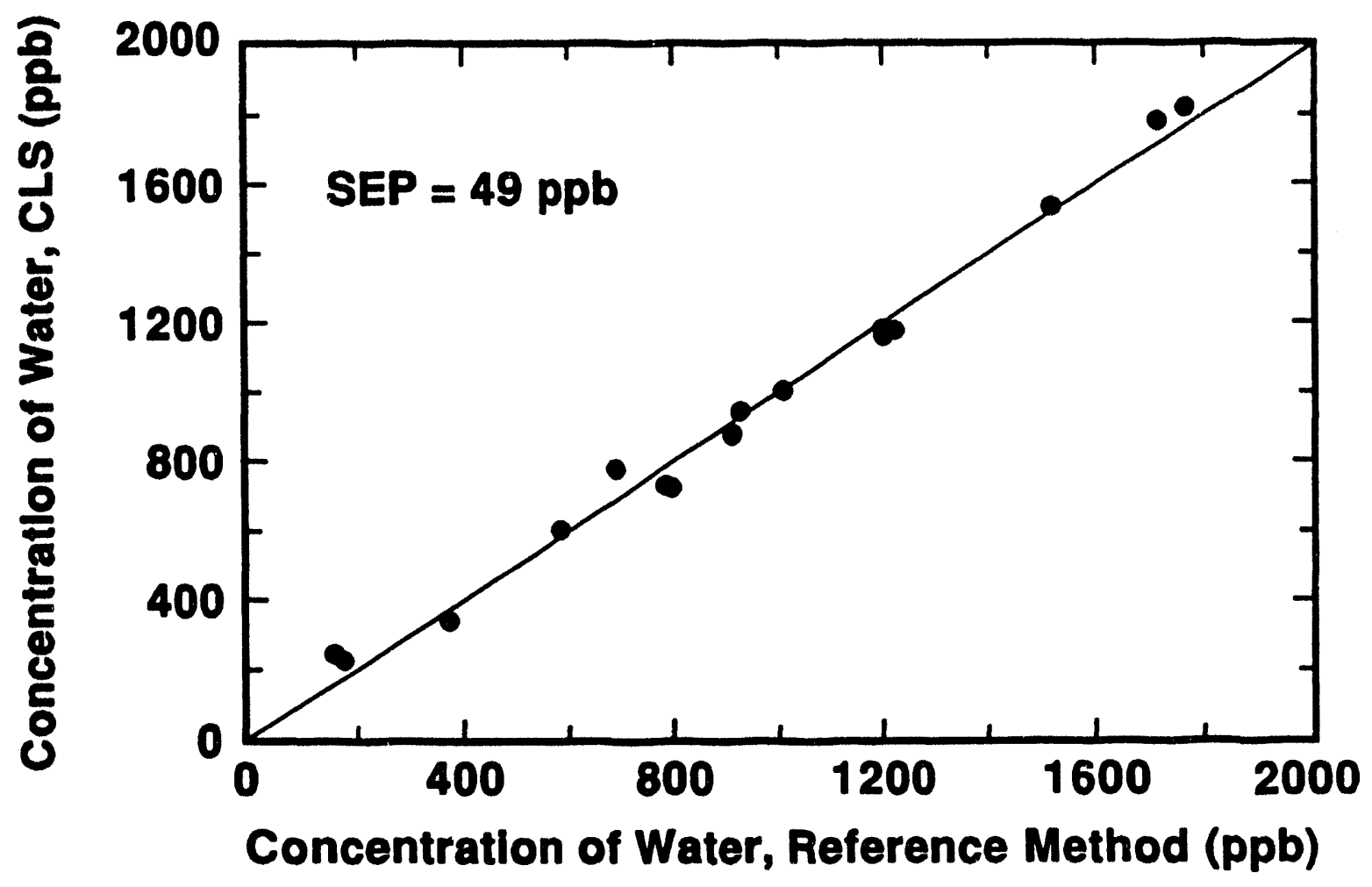

Fig. 32. The cross-validated prediction plot for the data in Fig. 29. 


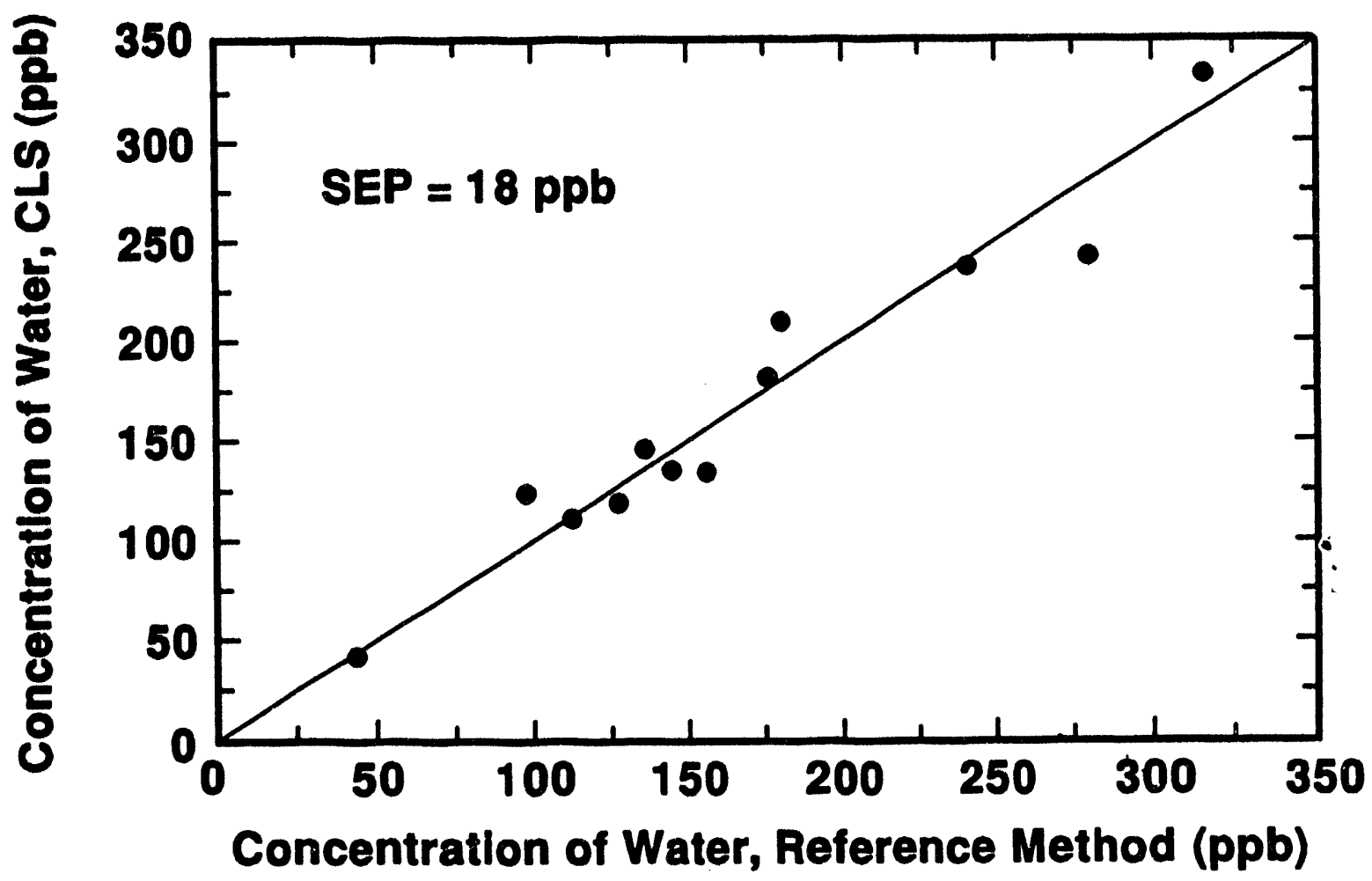

Fig. 33. The cross-validated prediction plot for the data in Fig. 30. 


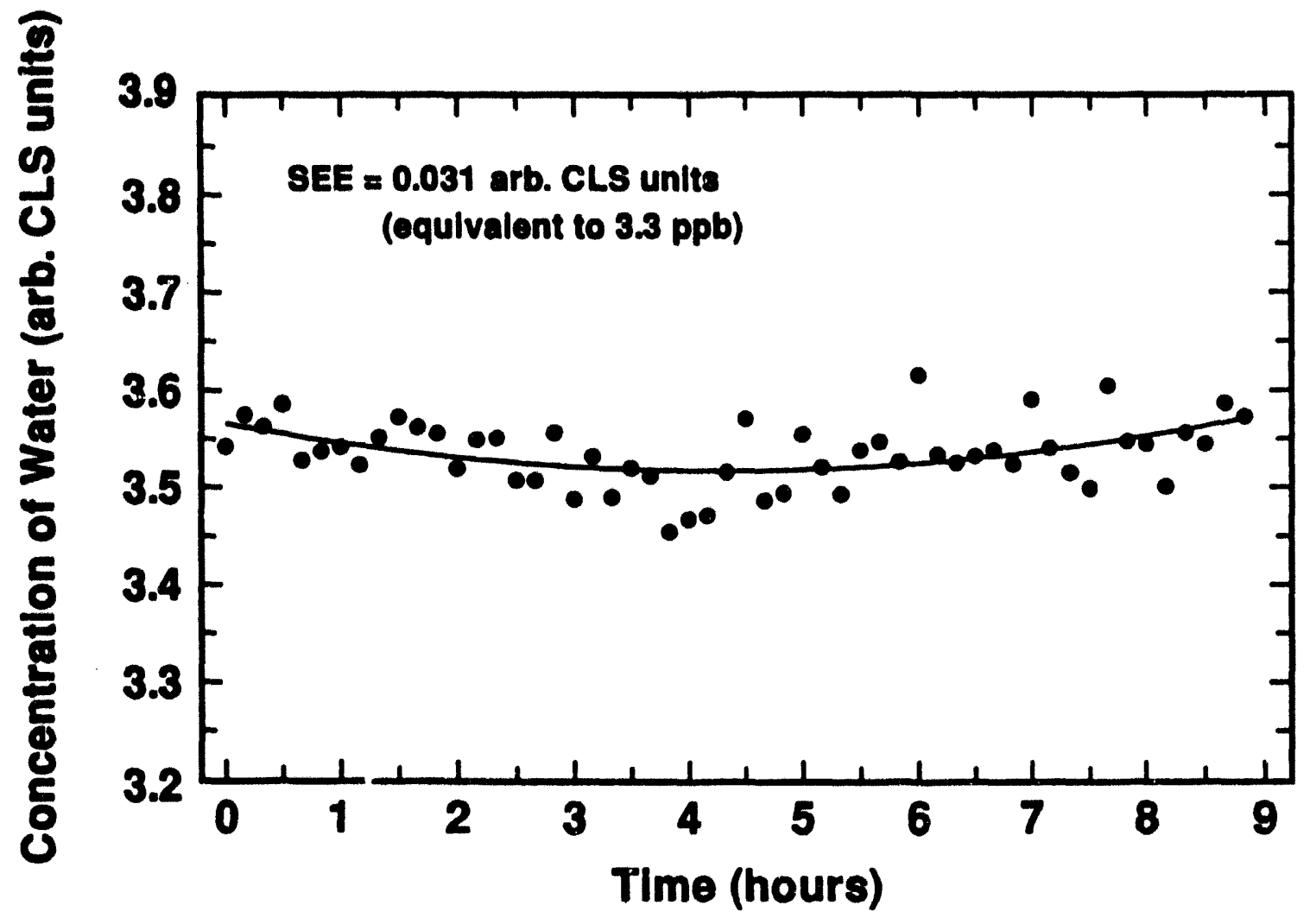

Fig. 34. Experimental data showing the precision of the measurement of the $\mathrm{H}_{2} \mathrm{O}$ concentration over a nine-hour period. The conditions are InSB detector, $\mathrm{N}_{2}$ carrier gas, and $8 \mathrm{~m}$ Axiom cell. The solid line is a second-order polynomial fit. 


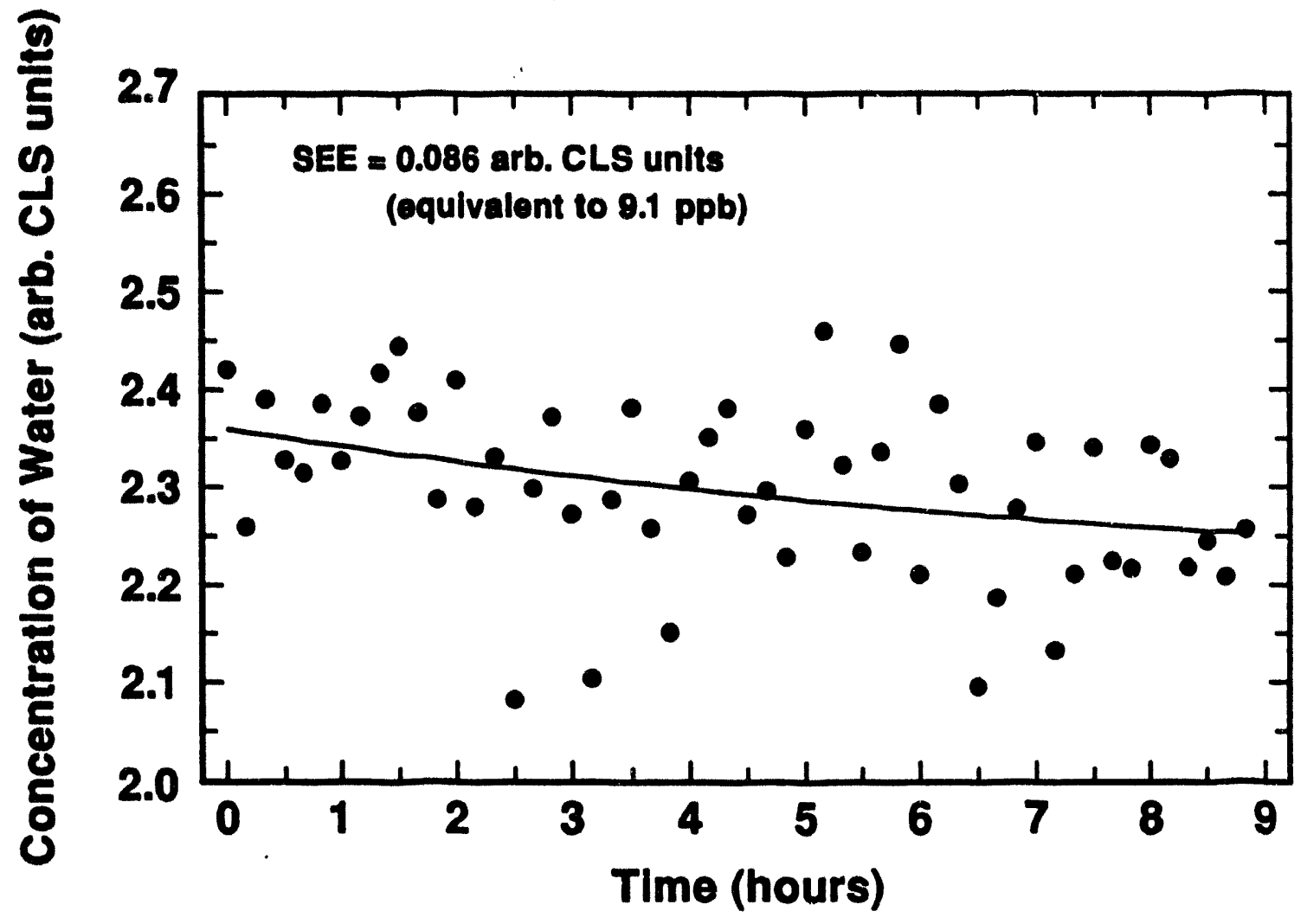

Fig. 35. Experimental data showing the precision of the measurement of the $\mathrm{H}_{2} \mathrm{O}$ concentration over a nine-hour period. The conditions are TGS detector, $\mathrm{N}_{2}$ carrier gas, and 8 $\mathrm{m}$ Axiom cell. The solid line is a second-order polynomial fit. 


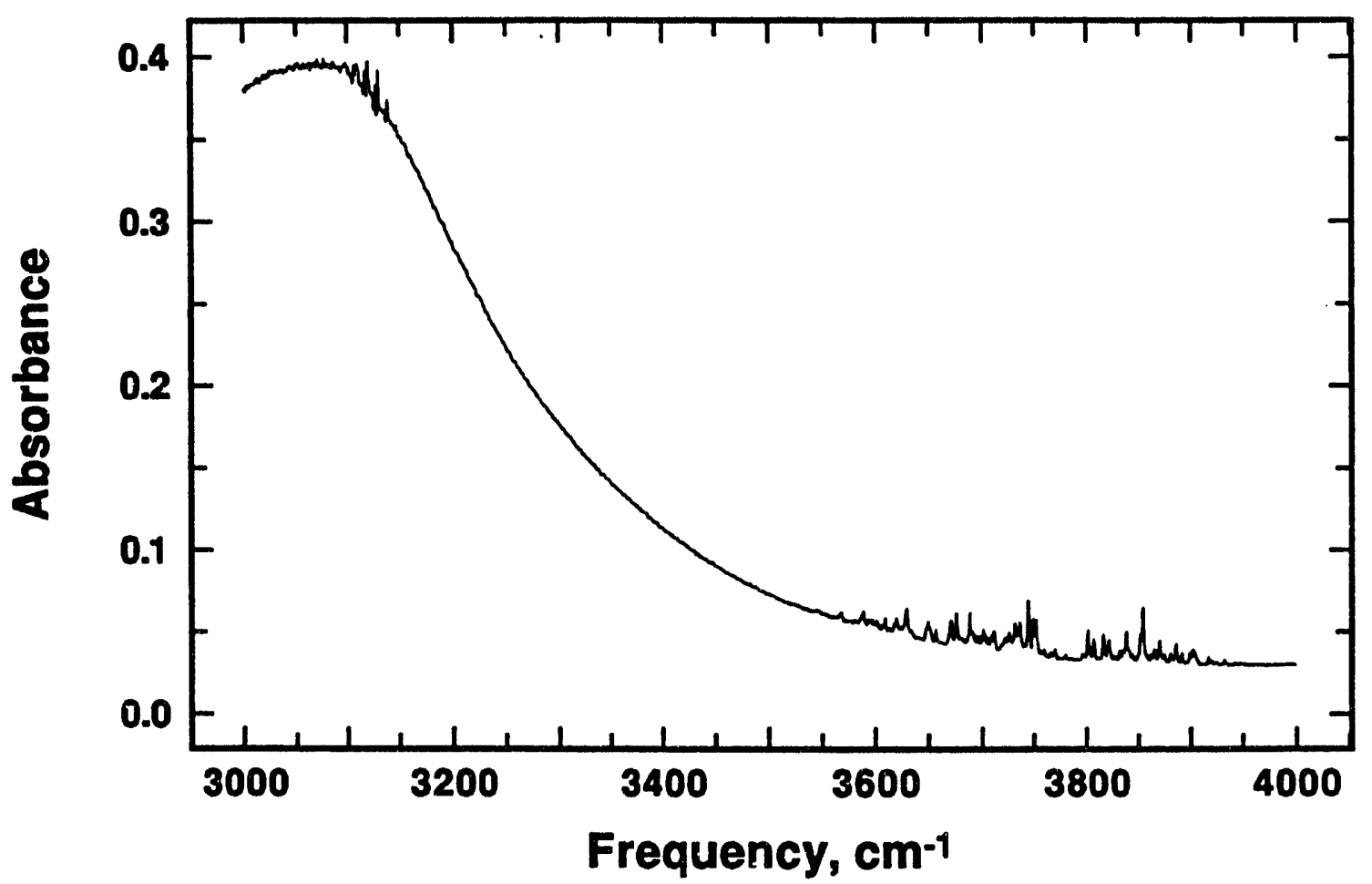

Fig. 36. Spectrum of $\mathrm{H}_{2} \mathrm{O}$ at about $10 \mathrm{ppm}$ in semiconductor grade $\mathrm{HCl}$. The strong $\mathrm{HCl}$ lines are merged by the spectrometer into a broad peak at $3000 \mathrm{~cm}^{-1}$. The $\mathrm{H}_{2} \mathrm{O}$ spectrum is at 3600 to $3900 \mathrm{~cm}^{-1}$. 


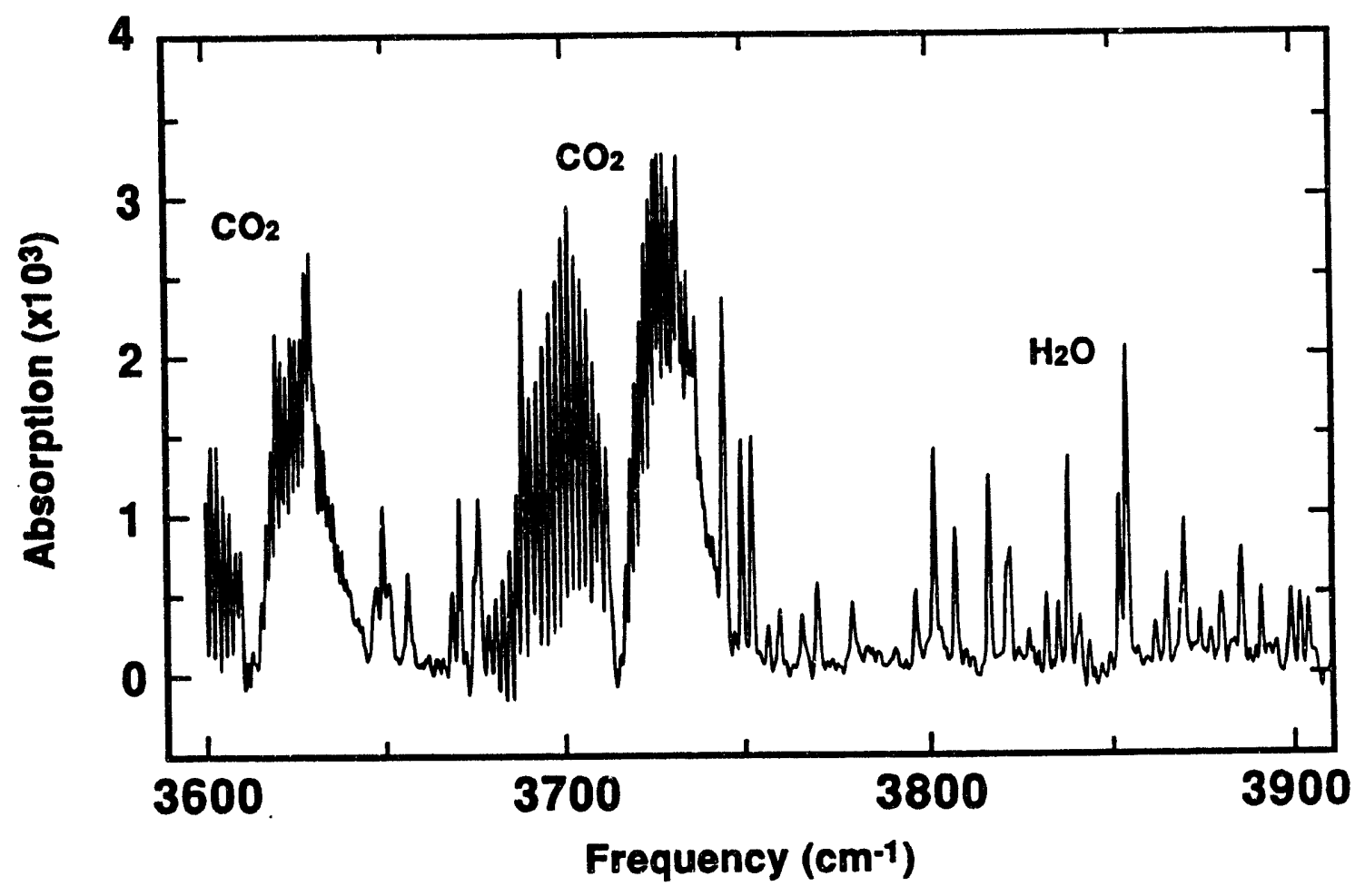

Fig. 37. Spectrum of $\mathrm{H}_{2} \mathrm{O}$ at about $500 \mathrm{ppb}$ in semiconductor grade $\mathrm{HCl}$. The spectral interference from the $\mathrm{CO}_{2}$ present at less than $10 \mathrm{ppm}$ is evident. 


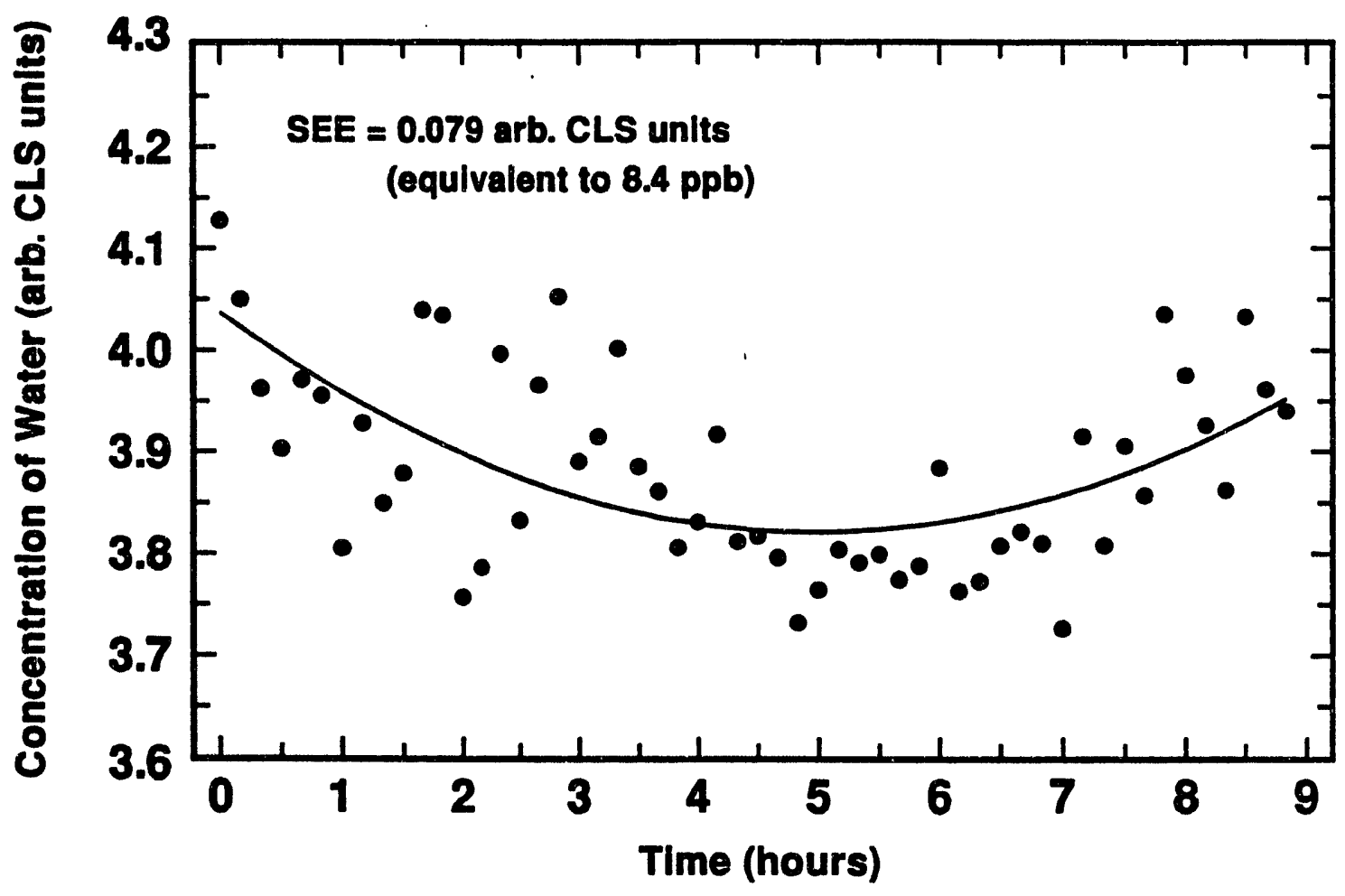

Fig. 38. Experimental data showing the precision of the measurement of the $\mathrm{H}_{2} \mathrm{O}$ concentration over a nine-hour period. The conditions are InSB detector, $\mathrm{HCl}$ carrier gas, and $8 \mathrm{~m}$ Axiom cell. The solid line is a second-order polynomial fit. 


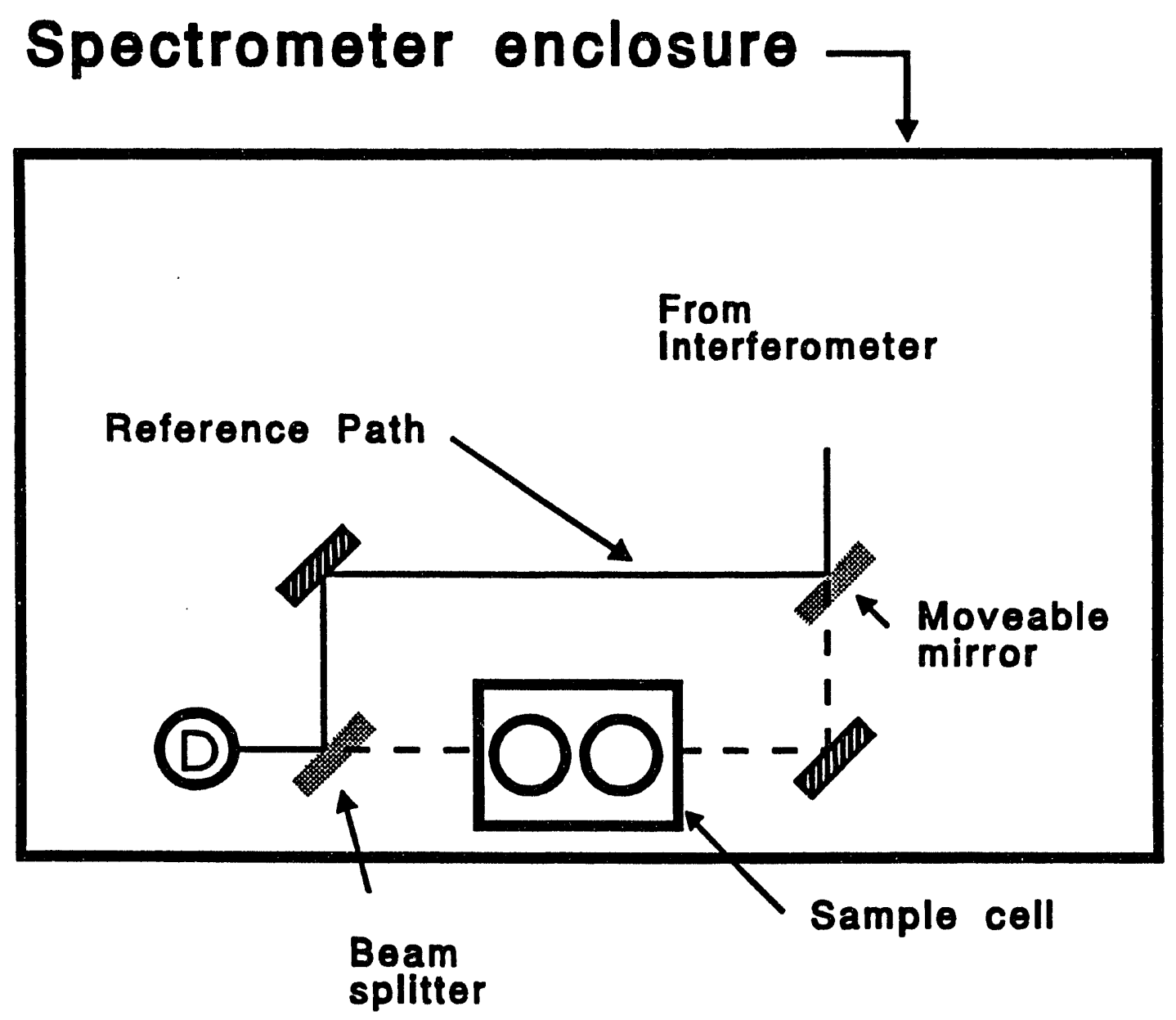

Fig. 39. Schematic of a portion of the redesigned detection system showing the concept of a dual-path measurement using a single detector. 

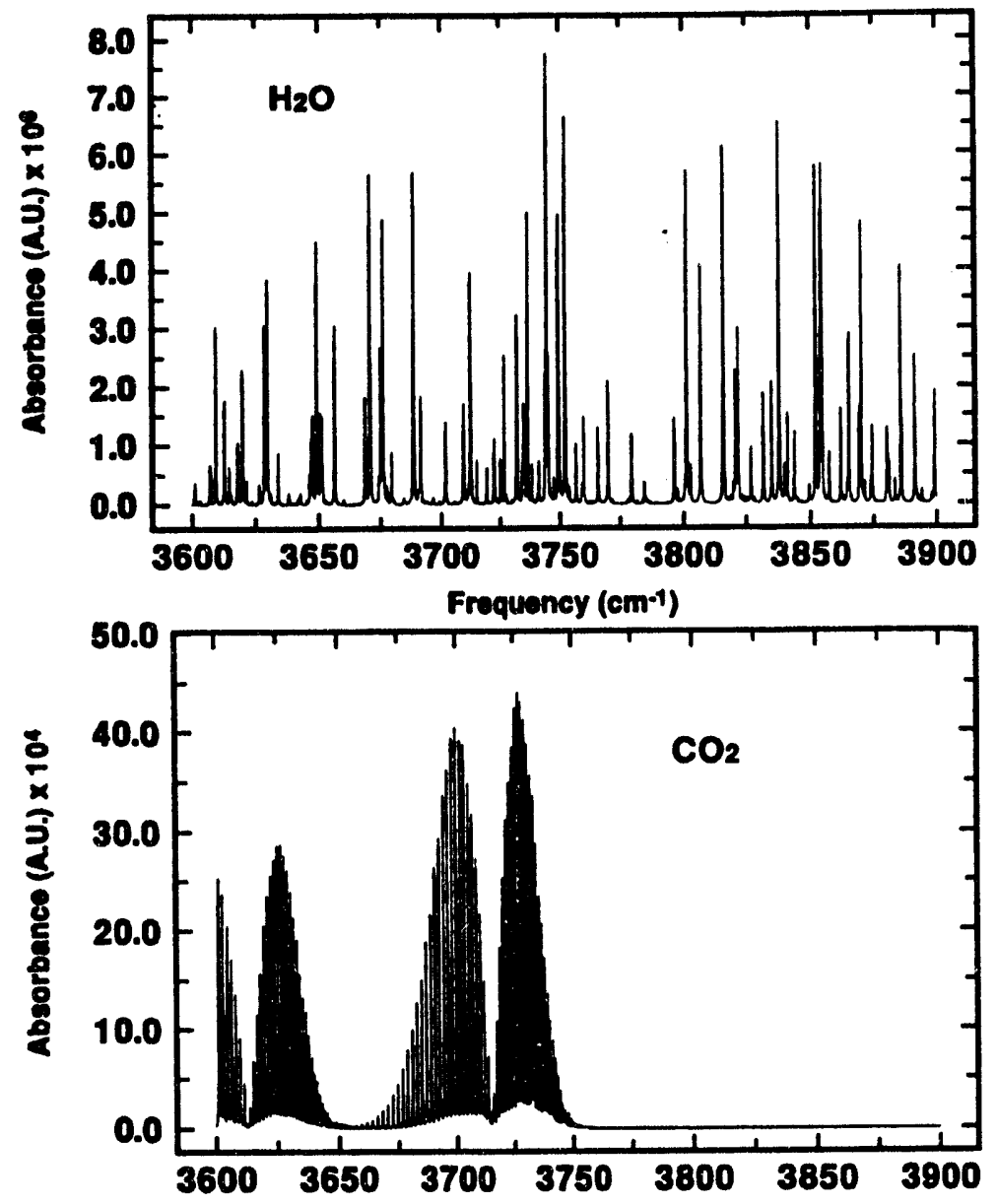

Frequency $\left(\mathrm{cm}^{-1}\right)$

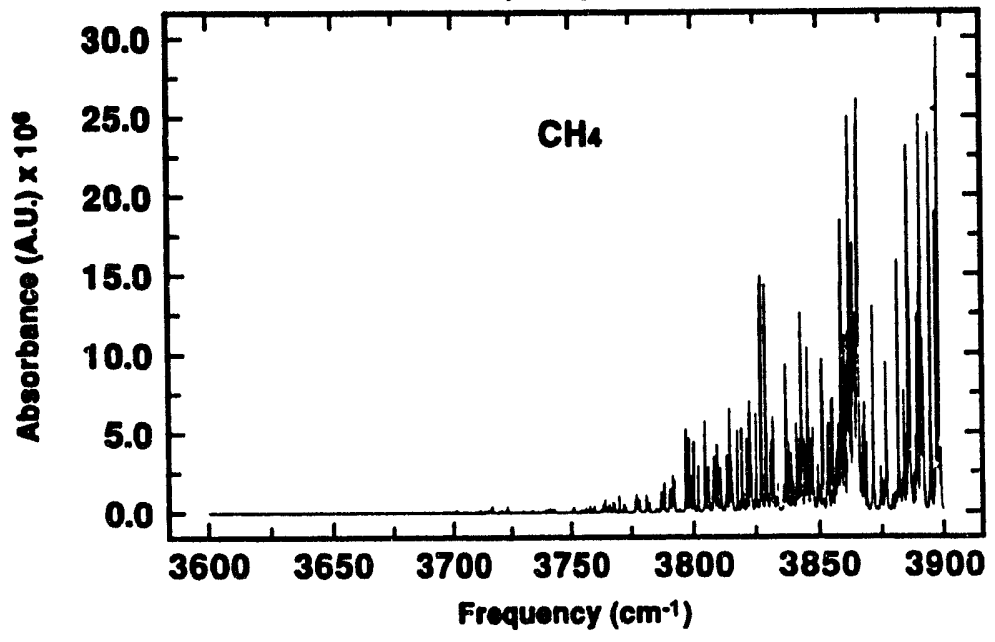

Fig. 40. Simulated absorption spectrum for $1 \mathrm{ppb}$ of $\mathrm{H}_{2} \mathrm{O}, 1 \mathrm{ppm}$ of $\mathrm{CO}_{2}$, and $1 \mathrm{ppm}$ of $\mathrm{CH}_{4}$. The spectra were generated from the HITRAN data base using a model of an FTIR spectrometer with $1 \mathrm{~cm}^{-1}$ resolution under the standard conditions for the present work. 


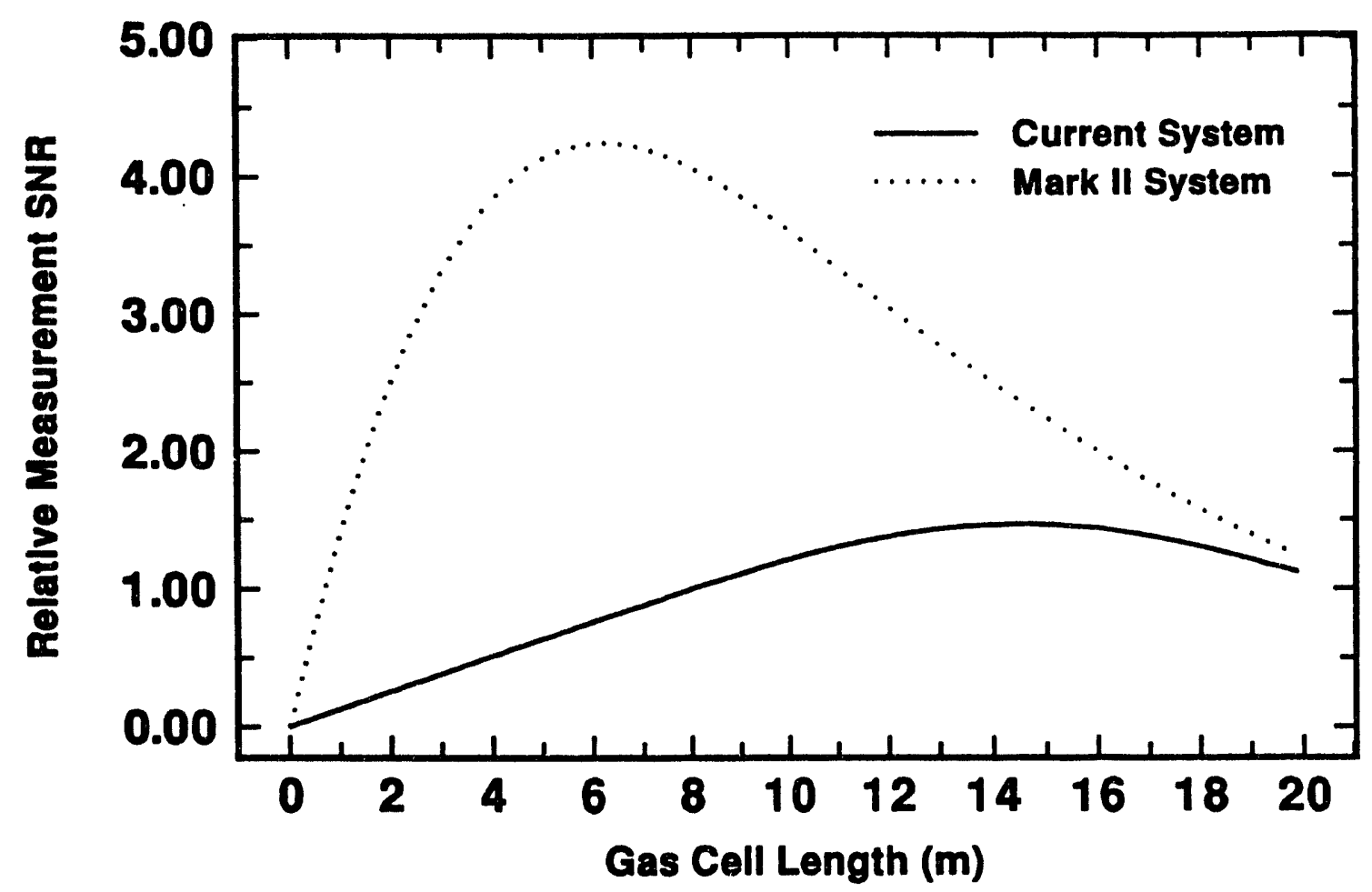

Fig. 41. Theoretical plot of the relative SNR as a function of the Axiom cell path length, according to Eq. 23 for the current system and the Mark II system. 


\section{DISTRIBUTION:}

1 L. H. Espinoza

Department of Chemistry

University of New Mexico

Albuquerque, NM 87106

1 T. M. Niemczyk

Department of Chemistry

University of New Mexico

Albuquerque, NM 87106

$\begin{array}{llll}10 & \text { MS } & 0343 & \text { B. R. Stallard, 1823 } \\ 1 & 0343 & \text { R. K. Rowe, 1823 } \\ 1 & 0343 & \text { M. J. Garcia, 1823 } \\ 1 & 0343 & \text { D. M. Haaland, 1823 } \\ 1 & 0343 & \text { J. A. Borders, 1823 } \\ 1 & 0337 & \text { A. Romig, 1800 } \\ 1 & 0342 & \text { R. P. Goehner, 1824 } \\ 1 & 1082 & \text { R. S. Blewer, 1305 } \\ 1 & 1078 & \text { C. W. Gwyn, 1302 } \\ 1 & 9018 & \text { Central Technical Files, 8523-2 } \\ 5 & 0899 & \text { Technical Library, 13414 } \\ 1 & 0619 & \text { Technical Publications, 13416 } \\ 10 & 0100 & \text { Document Processing for DOE/OSTI, 7613-2 }\end{array}$



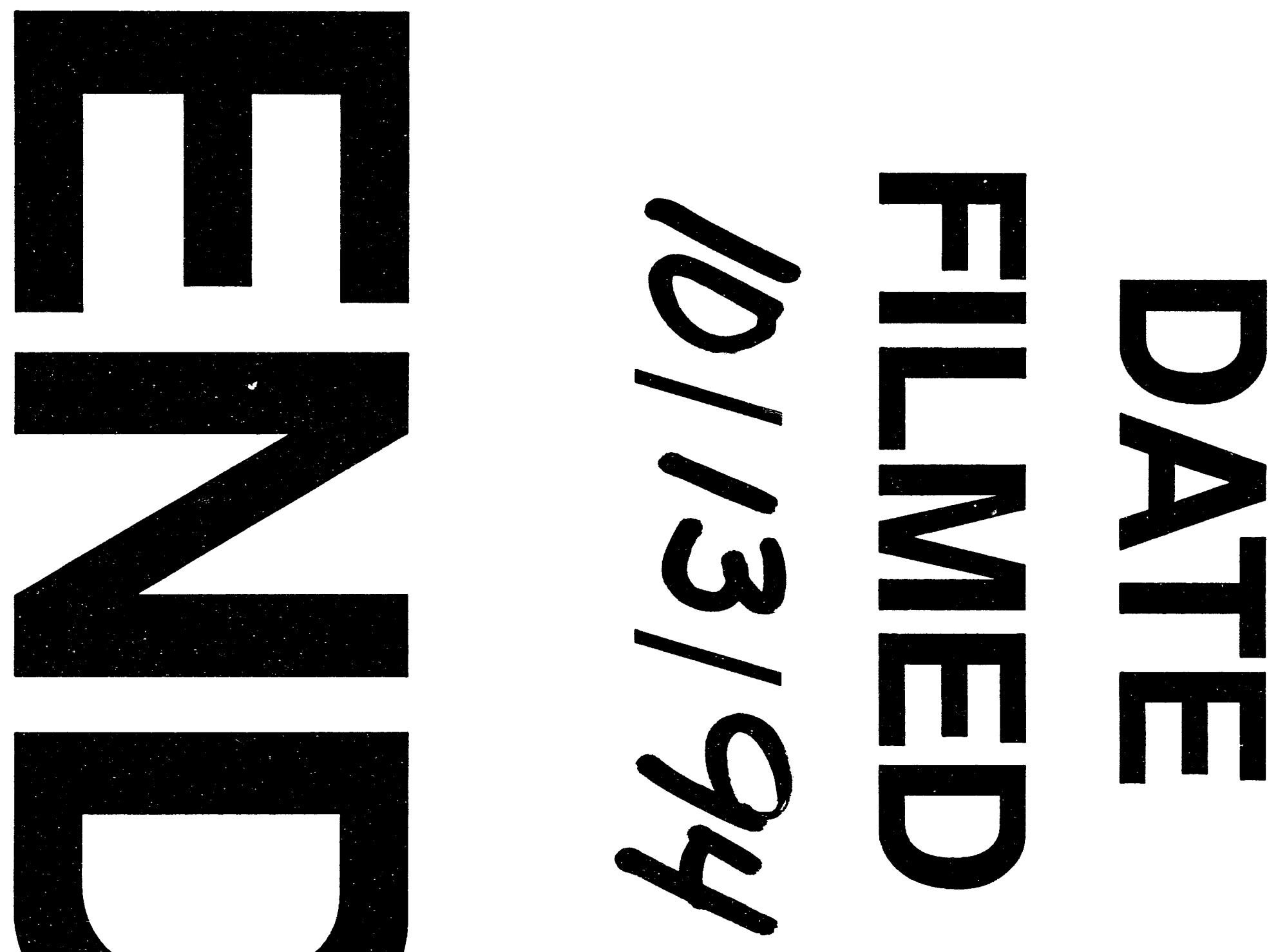
